\title{
A general Fredholm theory III: Fredholm functors and polyfolds
}

\author{
HELMUT HOFER \\ KRIS WYSOCKI \\ EDUARD ZEHNDER
}

\begin{abstract}
This is the third in a series of papers devoted to a general Fredholm theory in a new class of spaces, called polyfolds. We first introduce ep-groupoids and polyfolds. Then we generalize the Fredholm theory, which for $\mathrm{M}$-polyfolds has been presented in our paper [22], to the more general polyfold setting. The Fredholm theory consists of a transversality and a perturbation theory. The results form the basis for our application to Symplectic Field Theory.
\end{abstract}

58B99, 58C99; 46T99, 57R17

\section{Introduction and survey of results}

This paper is the third in a series of papers devoted to a generalized Fredholm theory. In Part I [21], the "splicing-based differential geometry" is developed. In this theory the familiar local models for spaces (open subsets of finite-dimensional spaces or Banach spaces) are replaced by more general local models, namely by open subsets of so-called splicing cores. Further, the notion of smoothness, as well as the notion of a smooth map, is generalized from the standard notion in finite dimensions to infinite dimensions in a new way. This generalization allows to extend the category of manifolds to a category of new smooth objects which open up the possibility to identify new structures in situations which could not be handled before. For example, the moduli space in Symplectic Field Theory (SFT) can be viewed as the zero-set of a Fredholm section (in a generalized sense) defined on bundles on spaces belonging to our new category. Under the appropriate transversality assumptions the solution sets are still "old-fashioned" manifolds or orbifolds and in bad cases branched manifolds or branched orbifolds. It still makes sense to talk about orientations in all cases and the structures suffice to establish a theory of integration in which Stokes' theorem holds true. This is all one needs in order to define invariants. 
Acknowledgments We would like to thank Peter Albers, Yakov Eliashberg, Eleny Ionel, Dusa McDuff and Tom Weinmann for very useful discussions. We thank the referee for valuable suggestions. While writing this paper $\mathrm{H}$ Hofer was partially supported by NSF grant DMS-0603957, K Wysocki was partially supported by NSF grant DMS-0606588, and E Zehnder was partially supported by TH-project.

\subsection{Background}

If one uses the new local models, the recipe for defining manifolds produces so-called M-polyfolds which are the starting point for further developments. In the following we assume the reader to be familiar with the concepts and results in [21]. More specifically, we assume familiarity with the notion of an $\mathrm{M}$-polyfold, its degeneration index, the notion of a strong bundle splicing and that of a filled section. Moreover, with the notion of an $\mathrm{sc}^{+}$-section of a strong $\mathrm{M}$-polyfold bundle, the notion of linearization, the notion of being linearized Fredholm, and the definition of the Fredholm index for an sc-Fredholm operator.

In the first part of the present paper we shall develop the theory of ep-groupoids and polyfolds. We shall modify the approach to orbifolds based on étale proper Lie groupoids, as presented in Moerdijk [28] and Moerdijk and Mrčun [29], and replace étale proper Lie groupoids by ep-groupoids which are based on M-polyfolds. In the second part of the paper we generalize the Fredholm theory in M-polyfolds from [22] to a theory of Fredholm functors and polyfold Fredholm sections. Fredholm functors can be viewed as Fredholm sections compatible with local symmetries represented by the morphisms. In general, it is not possible to bring a Fredholm functor into a general position by a functorial perturbation. However, if we allow multivalued perturbations are used this becomes possible. The price to pay is that the solution sets in case of transversality are neither manifolds nor orbifolds, but merely weighted branched submanifolds or suborbifolds. Nevertheless these objects have enough structure in order to establish a well-defined integration theory for so-called sc-differential forms as demonstrated in our preprint [20]. The integration on a branched ep-subgroupoid in [20] is used to construct invariants. Our paper extends some of the ideas from Cieliebak, Mundet i Riera and Salamon [2] to the functorial setting and it is also related to the recent work by McDuff [26]. The results of the present paper form the basis for the application to SFT given in our future works $[15 ; 16]$.

\subsection{Survey of the main results}

We now describe some of the main results postponing the precise definitions to the later sections. A manifold is a second countable paracompact space with an additional 
structure (the overhead) of equivalence classes of atlases of smoothly compatible charts. One can formalize the equivalence of atlases in a category-theoretic way as some kind of Morita-equivalence. From this point of view the step to an orbifold structure on a topological space is small. Here again we have an underlying topological space equipped with an the overhead of a Morita-equivalence class of étale proper Lie groupoids as described by Moerdijk [28]. Polyfolds are second countable paracompact spaces with an overhead of Morita-equivalence classes of étale proper M-polyfold groupoids. An étale proper M-polyfold groupoid, in short an ep-groupoid, generalizes the notion of an étale proper Lie groupoid. In particular, an ep-groupoid $Q$ is a category and $|Q|$ denotes the orbit space in which two objects are identified if there is a morphism between them. In the polyfold theory the role of an atlas for a manifold is played by a pair $(Q, \alpha)$ in which $Q$ is an ep-groupoid and $\alpha:|Q| \rightarrow Z$ is a homeomorphism between an orbit space $|Q|$ and a polyfold $Z$. However, one needs the whole ep-groupoid to encode the smooth structure on the polyfold.

In the following description of the main results we ignore the all important overhead and just note that it defines a smooth structure (in some new sense) on an otherwise second countable paracompact space $Z$. The topological space $Z$ equipped with this for the moment suppressed additional structure is called a polyfold. If one accepts a polyfold as a generalization of a (possibly infinite-dimensional) orbifold the results surveyed in the following appear familiar. In fact our results can be viewed as generalizations of known results in Banach manifolds to much more general spaces.

It is a part of its structure that a polyfold $Z$ is equipped with a filtration

$$
Z=Z_{0} \supset Z_{1} \supset Z_{2} \supset \cdots \supset Z_{i} \supset Z_{i+1} \supset \cdots \supset Z_{\infty}:=\bigcap_{i \geq 0} Z_{i}
$$

into subsets $Z_{i}$ of $Z$ which have topologies such that the inclusions $Z_{i+1} \rightarrow Z_{i}$ are continuous and enjoy some compactness properties. Moreover, $Z_{\infty}$ is dense in every space $Z_{i}$. In fact every $Z_{m}$ carries some smooth structure (again in the new sense) as well and smooth maps between polyfolds have to preserve these levels of smoothness. We can define strong polyfold bundles $p: W \rightarrow Z$. The space $W$ carries a double integer-filtration $W_{n, k}$ for $0 \leq k \leq n+1$, where we may view $k$ as the fiber regularity. A smooth section $f$ of the bundle $p: W \rightarrow Z$ maps $Z_{m}$ to $W_{m, m}$. The collection of smooth sections is denoted by $\Gamma(p)$. There is an additional class of so-called $\mathrm{sc}^{+}$-sections. They are smooth sections mapping $Z_{m}$ to $W_{m, m+1}$. Due to a compactness property of the fiberwise embeddings $W_{m, m+1} \rightarrow W_{m, m}$, the space of $\mathrm{sc}^{+}$-sections can be viewed as a well-defined universe of compact perturbations. Of particular importance will be the set of $\mathrm{sc}^{+}$-multisections. An $\mathrm{sc}^{+}$-multisection can be identified with a functor $\lambda: W \rightarrow \mathbb{Q}^{+}$such that near every point $z \in Z$ there exist a 
finite number of $\mathrm{sc}^{+}$-sections $s_{i}$ for $i \in I$, and associated positive rational weights $\sigma_{i}$ satisfying for $w \in W$,

$$
\lambda(w)=\sum_{\left\{i \in I \mid s_{i}(p(w))=w\right\}} \sigma_{i} .
$$

The sum over the empty set is defined to be zero. As it turns out two such sc ${ }^{+}-$ multisections $\lambda$ and $\tau$ can be added resulting in the $\operatorname{sc}^{+}$-multisection $\lambda \oplus \tau$. Our notion of multisections generalizes ideas in Cieliebak, Mundet i Riera and Salamon [2] (where group actions were studied) to a functorial context. In [22] we have introduced Fredholm sections which now will be generalized to the polyfold context. For a pair $(f, \lambda)$ in which $f$ is a Fredholm section and $\lambda$ an $\mathrm{sc}^{+}$-multisection, we define the solution set $S(f, \lambda)$ to be the set

$$
\begin{aligned}
S(f, \lambda) & :=\{z \in Z \mid \lambda(f(z))>0\} . \\
\text { By } & S(f):=\{z \in Z \mid f(z)=0\}
\end{aligned}
$$

we shall denote the solution set of the Fredholm section $f: Z \rightarrow W$ where 0 is the zero section of the bundle $p$. We should point out that the fiber of a strong polyfold bundle does not have a linear structure but it has a preferred section 0 . We also define the notion of an auxiliary norm $N: W_{0,1} \rightarrow \mathbb{R}^{+}$in order to measure the size of an $\mathrm{sc}^{+}-$section or $\mathrm{sc}^{+}$-multisection. (It is not a real norm but related to a norm in the overhead).

Theorem 1.1 (Compactness) Let $p: W \rightarrow Z$ be a strong polyfold bundle possibly with boundary with corners and let $f$ be a proper Fredholm section of $p$. Then, given an auxiliary norm $N$ for the strong bundle $p$, there exists an open neighborhood $U$ of the solution set $S(f)$ so that the solution set $S(f, \lambda)$ is compact for every $\mathrm{sc}^{+}$-multisection $\lambda$ having its support in $U$ and satisfying $N(\lambda) \leq 1$.

We should point out that the boundary of a polyfold has very little to do with the set theoretic boundary of the underlying topological space (whatever it means in any given context). For example, the subspace $Z$ of $\mathbb{R}^{2}$ given by

$$
Z=((-\infty, 0) \times \mathbb{R}) \cup([0,1] \times\{0\})
$$

with the induced topology admits a smooth polyfold structure without boundary. (One might think that $(1,0)$ is a boundary point!)

The next result shows that we can perturb the multisections to obtain as a solution set a branched suborbifold of $Z$. The advantage is that we can integrate over branched suborbifold, once they are equipped with an orientation, as is demonstrated in [20]. 
Theorem 1.2 (Perturbation) Let $f$ be a proper (oriented) Fredholm section of the strong polyfold bundle $p: W \rightarrow Z$ with boundary with corners and assume that the polyfold structure for $Z$ is built on separable Hilbert spaces. Let $N$ be an auxiliary norm for $p$ and $U$ an open neighborhood of the solution set $S(f)=\{z \in Z \mid f(z)=0\}$ so that the pair $(N, U)$ controls compactness. Then for every sc ${ }^{+}$-multisection $\lambda$ having support in $U$ and satisfying $N(\lambda)<\frac{1}{2}$ and every $\varepsilon \in\left(0, \frac{1}{2}\right)$, there exists an $\mathrm{sc}^{+}$-multisection $\tau$ having support in $U$ and satisfying $N(\tau)<\varepsilon$, so that the pair $(f, \lambda \oplus \tau)$ is in general position. In particular, the solution set $S(f, \lambda \oplus \tau)$ is an (oriented) compact branched suborbifold with boundary with corners. The solution set $S(f, \lambda \oplus \tau)$ is equipped with the natural weight function $w: S(f, \lambda \oplus \tau) \rightarrow(0, \infty) \cap \mathbb{Q}$ defined by

$$
w(z)=(\lambda \oplus \tau)(f(z))
$$

A branched suborbifold is essentially a pair $(S, w)$ in which $S$ is a subset of $Z$ as the above set $S(f, \lambda \oplus \tau)$ and $w$ is a map associating with a point in $S$ a positive rational weight. If $f$ is a proper Fredholm section and $\lambda_{i}, i=0,1$, are generic $\mathrm{sc}^{+}-$multisections, then there exists a generic family $\lambda_{t}$ interpolating between them so that the solution set $\left\{(t, z) \in[0,1] \times Z \mid \lambda_{t}(f(z))>0\right\}$ is a smooth branched suborbifold with boundary with corners interpolating between the solution sets $S_{0}$ and $S_{1}$. Next assume $\partial Z=\varnothing$. Using determinant bundles one can introduce the notion of an orientation $\mathfrak{o}$ for a Fredholm section $f$ leading to the notion of an oriented Fredholm section $(f, \mathfrak{o})$. We summarize the results needed in the Appendix, Section 5.4. The constructions of determinant bundles are in principle well-known (see for example Donaldson and Kronheimer [4] and Floer and Hofer [6]), however, in the polyfold context they are not entirely standard since the linearized Fredholm sections do not depend continuously as operators on the points at which they are linearized. This requires some extra work carried out in [17]. Transversal pairs $(f, \lambda)$, pairs in good position, and pairs in general position occurring in the following result are defined in Definition 4.7 below.

Theorem 1.3 (Invariants) Let $f$ be a proper oriented Fredholm section of the strong polyfold bundle $p: W \rightarrow Z$ without boundary and assume that the polyfold structure for $Z$ is based on separable Hilbert spaces. Assume that $N$ is an auxiliary norm for $p$ and $U$ an open neighborhood of the solution set $S(f)$ so that the pair $(N, U)$ controls compactness. Then there is a well-defined map

$$
\Phi_{f}: H_{\mathrm{dR}}^{*}(Z, \mathbb{R}) \rightarrow \mathbb{R}
$$

defined on the deRham cohomology group $H_{\mathrm{dR}}^{*}(Z, \mathbb{R})$ and having the following property. For any sc ${ }^{+}$-multisection $\lambda$ having support in $U$ and satisfying $N(\lambda)<1$ so that 
$(f, \lambda)$ is a transversal pair (in particular, the associated solution set $S=S(f, \lambda)$ is an oriented compact branched suborbifold), the map $\Phi_{f}$ is represented by the formula

$$
\Phi_{f}([\omega])=\int_{(S, w)} \omega=\mu_{\omega}^{(S, w)}(S) .
$$

The weight function $w: S \rightarrow \mathbb{Q}^{+}$is defined by $w(z)=\lambda(f(z))$. Moreover, if $t \rightarrow f_{t}$ is an sc-smooth proper oriented homotopy of Fredholm sections, then

$$
\Phi_{f_{0}}=\Phi_{f_{1}} .
$$

Here $\mu_{\omega}^{(S, w)}$ is a natural signed measure associated with an sc-differential form $\omega$ on the polyfold $Z$ and the branched suborbifold $(S, w)$ (of course the measure can only be nonzero provided the degree of the form and the dimension of $S$ match). The underlying measure space is $(S, \mathcal{L}(S))$. The $\sigma$-algebra $\mathcal{L}(S)$ of subsets of $S$ is a natural generalization of the Lebesgue $\sigma$-algebra on a smooth manifold. We refer to [20] for more details.

For example, as sketched in Section 1.3, the disjoint union of the Gromov-compactified moduli spaces of pseudoholomorphic curves with varying arithmetic genus and representing the various second homology classes in a compact symplectic manifold $(Q, \omega)$ can be viewed as the zero set of a Fredholm section $f$ of some strong polyfold bundle $p: W \rightarrow Z$, which in every connected component is proper. The evaluation map ev $\mathrm{ev}_{l}: Z \rightarrow Q$ at the $l$-marked point is smooth (in the new sense) and pulls back every differential form on $Q$ to an sc-differential form $\omega$ on $Z$. Also the map which associates with a point in $Z$ the underlying stable part of the domain defines a smooth map into the Deligne-Mumford stack and pulls back differential forms on the stack to sc-differential forms $\omega$ on $Z$. Wedges of suitable forms can be integrated over a (generic) branched suborbifold which is the solution set for a suitable $\mathrm{sc}^{+}-$multisection perturbation, and organizing the data in the usual way we obtain the GW-potential as defined in McDuff and Salamon [27]. That the GW-theory fits into our framework will be shown in detail in [15], though the main point of [15] is the construction of the polyfold structures in the presence of nodes. The understanding of the nodes presents already all the analytical difficulties related to the phenomenon called breaking of trajectories occurring, for example, in Floer theory and in SFT.

Using the previous theorem one can associate with a proper Fredholm section a $\mathbb{Q}$ valued degree as follows. If $f$ is an oriented proper Fredholm section of Fredholm index 0 we can integrate the class $[1] \in H_{\mathrm{dR}}^{0}(Z, \mathbb{R})$, ie the cohomology class of the constant 1 -function. Then we define the degree of $f$ by

$$
\operatorname{deg}(f)=\Phi_{f}([1]) .
$$


This degree takes, as one can show, values in $\mathbb{Q}$ and has the usual properties of a degree.

There is also a version for the boundary case. Consider the inclusion $j: \partial Z \rightarrow Z$ which restricted to the local faces is sc-smooth. We can introduce the notion of an sc-differential form on $\partial Z$ by taking sc-differential forms on the local faces which are compatible at their intersections. Let us denote by $\Omega^{k}(j)$ the collection of pairs $(\omega, \tau) \in \Omega^{k}(Z) \oplus \Omega^{k-1}(\partial Z)$. The Cartan derivative $d$ is defined as $d(\omega, \tau)=$ $\left(d \omega, j^{*} \omega-d \tau\right)$ and the associated deRham cohomology group $H_{\mathrm{dR}}^{*}(j)$ is denoted by $H_{\mathrm{dR}}^{*}(Z, \partial Z)$.

Theorem 1.4 (Invariants in case of boundary) Let $f$ be a proper oriented Fredholm section of the strong polyfold bundle $p: W \rightarrow Z$ with boundary with corners and assume that the polyfold structure for $Z$ is built on separable Hilbert spaces. Assume that $N$ is an auxiliary norm for $p$ and $U$ an open neighborhood of the solution set $S(f)$ so that the pair $(N, U)$ controls compactness. Then there exists a well-defined map

$$
\Psi_{f}: H_{\mathrm{dR}}^{*}(Z, \partial Z) \rightarrow \mathbb{R}
$$

having the following property. For any $\mathrm{sc}^{+}-$multisection $\lambda$ having support in $U$ and satisfying $N(\lambda)<1$ so that $(f, \lambda)$ is in general position (in particular, the associated solution set $S(f, \lambda)$ is an oriented compact branched suborbifold with boundary with corners), the map $\Psi_{f}$ has the representation

$$
\Psi_{f}([\omega, \tau]):=\int_{\left(S(f, \lambda), \lambda_{f}\right)} \omega-\int_{\left(\partial S(f, \lambda), \lambda_{f}\right)} \tau .
$$

Moreover, if $t \mapsto f_{t}$ is an sc-smooth proper oriented homotopy of Fredholm sections, then

$$
\Psi_{f_{0}}=\Psi_{f_{1}} .
$$

As already mentioned there are approaches to the structure of moduli spaces of stable maps which are different from ours. In some approaches so-called Kuranishi structures are used; see Fukaya and Ono [8], Fukaya, Oh, Ohta and Ono [7] and Lu and Tian [24]. We would like to point out that it is quite straightforward to construct a "forgetful" functor from the polyfold Fredholm theory to a class of Kuranishi structures; see Hofer [14].

\subsection{Sketch of the application to Gromov-Witten}

As an illustration of the new concepts in this paper, we sketch an application to the Gromov-Witten (GW)-invariants referring to [15] for the details and the proofs. The 
GW-invariants are invariants of symplectic manifolds deduced from the structure of stable pseudoholomorphic maps from Riemann surfaces to the symplectic manifold. The construction of GW-invariants for general symplectic manifolds goes back to Fukaya and Ono [8] and Li and Tian [23]. Earlier work for special symplectic manifolds are due to Ruan [31; 30]. Recently, Cieliebak and Mohnke [1] proved the genus zero case using Donaldson's theory [3] of codimension two symplectic hypersurfaces in order to establish the crucial transversality property. Our approach is quite different. As a consequence of general principles, compact smooth moduli spaces are produced over which one can integrate. In the GW-case, the theory applies for arbitrary genus and since the theory also solves all the smoothness problems automatically, many results, as for example the composition formula for the GW-invariants, do not require extra work.

We start our sketch recalling some concepts from the theory of Riemann surfaces. Nodal Riemann surfaces show up in the compactification of the moduli space of compact Riemann surfaces $S$. A nodal Riemann surface is a multiplet

$$
(S, j, M, D)
$$

in which the pair $(S, j)$ is a closed, not necessarily connected, Riemann surface $S$ equipped with the complex structure $j$. So, $S$ is the disjoint union of finitely many connected compact Riemann surfaces. The set $M \subset S$ is an ordered finite subset of points, called marked points. The unordered set $D$ consists of finitely many unordered pairs $\{x, y\}$ of points in $S$ satisfying $x \neq y$ and called nodal pairs. Moreover, $\{x, y\} \cap\left\{x^{\prime}, y^{\prime}\right\} \neq \varnothing$ implies that the two sets are equal. The points of a nodal pair may belong to the same component or to different components of $S$. Denoting by $|D|$ the collection of all the points of $S$ contained in the set of nodal pairs $D$, we assume, in addition, that $|D| \cap M=\varnothing$. We call $|D|$ the nodal set and the points in $|D|$ the nodal points. A special point of the nodal Riemann surface is a point in $S$ which is either a nodal point or a marked point. The nodal Riemann surface $(S, j, M, D)$ is called connected if the topological space obtained by identifying $x \equiv y$ for all nodal pairs $\{x, y\} \in D$ is a connected space. In this terminology it is possible that the nodal surface $(S, j, M, D)$ is connected but the Riemann surface $S$ has several connected components $C$, namely its domain components. The arithmetic genus $g_{\text {a }}$ agrees with the genus of the connected compact Riemann surface obtained by properly gluing the components $C$ of $S$ at all the nodes. The arithmetic genus $g_{\text {a }}$ of the connected nodal Riemann surface $(S, j, M, D)$ is the nonnegative integer $g_{\text {a }}$ defined by

$$
g_{\mathrm{a}}=1+\sharp D+\sum_{C}(g(C)-1)
$$


where $\sharp D$ is the number of nodal pairs in $D$ and where the sum is taken over the finitely many domain components $C$ of the Riemann surface $S$. Two connected nodal Riemann surfaces

$$
(S, j, M, D) \quad \text { and } \quad\left(S^{\prime}, j^{\prime}, M^{\prime}, D^{\prime}\right)
$$

are called isomorphic, if there exists a biholomorphic map

$$
\varphi:(S, j) \rightarrow\left(S^{\prime}, j^{\prime}\right),
$$

(ie, the map $\varphi$ satisfies $T \varphi \circ j=j^{\prime} \circ T \varphi$ ), mapping marked points onto marked points (preserving the ordering) and nodal pairs onto nodal pairs. If the two nodal Riemann surfaces are identical, the isomorphism above is called an automorphism of the nodal surface $(S, j, M, D)$. In the following we denote by

$$
[(S, j, M, D)]
$$

the equivalence class of all connected nodal surfaces isomorphic to the connected nodal Riemann surface $(S, j, M, D)$.

A crucial role play the so-called stable nodal surfaces.

Definition 1.5 The connected nodal Riemann surface $(S, j, M, D)$ is called stable, if the group of its automorphisms is finite.

One knows that a connected nodal Riemann surface $(S, j, M, D)$ is stable if and only if every connected domain component $C$ of $S$ satisfies

$$
2 g(C)+\sharp M_{C} \geq 3,
$$

where $g(C)$ is the genus of $C$ and where $M_{C}=C \cap(M \cup|D|)$ are the special points lying on $C$.

After having recalled the concepts from Riemann surface theory we start with the analytical set up of our approach to the GW-invariants. We consider a symplectic manifold $(Q, \omega)$ and assume, for simplicity, that $\partial Q=\varnothing$. We study maps $u: S \rightarrow Q$ defined on Riemann surfaces $S$ into the symplectic manifold having special regularity properties introduced below. By

$$
u: \mathcal{O}(S, z) \rightarrow Q
$$

we shall denote a germ around the point $z \in S$ defined on a piece of the Riemann surface $S$. 
Definition 1.6 Let $m \geq 2$ be an integer and $\delta>0$. The germ of a continuous map $u: \mathcal{O}(S, z) \rightarrow Q$ is called of class $(m, \delta)$ at the point $z$ if for a smooth chart $\varphi: U(u(z)) \rightarrow \mathbb{R}^{2 n}$ of $Q$ mapping $u(z)$ to 0 and for holomorphic polar coordinates $\sigma:[0, \infty) \times S^{1} \rightarrow S \backslash\{z\}$ around $z$, the map

$$
v(s, t)=\varphi \circ u \circ \sigma(s, t),
$$

which is defined for $s$ large, has partial derivatives up to order $m$, which if weighted by $e^{\delta s}$, belong to $L^{2}\left([R, \infty) \times S^{1}, \mathbb{R}^{2 n}\right)$ if $R$ is sufficiently large. The germ is called of class $m$ around the point $z \in S$, if $u$ belongs to the class $H_{\mathrm{loc}}^{m}$ near $z$.

The above definition does not depend on the choices involved, like charts on $Q$ and holomorphic polar coordinates on $S$.

We next consider multiplets

$$
\alpha:=(S, j, M, D, u)
$$

in which $(S, j, M, D)$ is a connected nodal Riemann surface and

$$
u: S \rightarrow Q
$$

is a continuous map.

Definition 1.7 Let $m \geq 2$ be an integer and $\delta>0$. The multiplet $\alpha=(S, j, M, D, u)$ is a stable map of class $(m, \delta)$, if it satisfies the following properties:

- The map $u$ is of class $(m, \delta)$ around the points belonging to the nodal set $|D|$ and of class $m$ around all the other points of $S$.

- For every nodal pair $\{x, y\} \in D, u(x)=u(y)$.

- If a connected component $C$ of $S$ has genus $g_{C}$ and $M_{C}$ special points, and satisfies $2 \cdot g_{C}+M_{C} \leq 2$, then

$$
\int_{C} u^{*} \omega>0
$$

Two stable maps $\alpha=(S, j, M, D, u)$ and $\alpha^{\prime}=\left(S^{\prime}, j^{\prime}, M^{\prime}, D^{\prime}, u^{\prime}\right)$ are called equivalent if there exists an isomorphism $\varphi:(S, j, M, D) \rightarrow\left(S^{\prime}, j^{\prime}, M^{\prime}, D^{\prime}\right)$ between the connected nodal Riemann surfaces satisfying

$$
u^{\prime} \circ \varphi=u \text {. }
$$

An equivalence class $[\alpha]=[(S, j, M, D, u)]$ is called a stable curve of class $(m, \delta)$.

The following space $Z$ will be equipped with a polyfold structure. 
Definition 1.8 Fix $\delta_{0} \in(0,2 \pi)$. The collection of all equivalence classes $[\alpha]=$ $[(S, j, M, D, u)]$ of class $\left(3, \delta_{0}\right)$ is called the space of stable curves into the symplectic manifold $Q$ of class $\left(3, \delta_{0}\right)$, and is denoted by $Z$.

In [15] the following result is proved for the set $Z$ of the stable curves of class $\left(3, \delta_{0}\right)$.

Theorem 1.9 (1) The space $Z$ has a natural second countable paracompact topology.

(2) Given a strictly increasing sequence $\left(\delta_{m}\right)$ starting at the previously chosen $\delta_{0}$ and staying below $2 \pi$ and given the exponential gluing profile $\varphi:(0,1] \rightarrow[0, \infty)$ defined by $\varphi(r)=e^{1 / r}-e$, the space $Z$ has a natural polyfold structure where the $m$-th level consists of equivalence classes $[(S, j, M, D, u)]$ in which $u$ is of class $\left(m+3, \delta_{m}\right)$.

Formulated in the technical terms introduced below, given the sequence $\left(\delta_{i}\right)$ and the exponential gluing profile $\varphi$, there exists a natural collection of pairs $(X, \beta)$ in which $X$ is an ep-groupoid and $\beta:|X| \rightarrow Z$ is a homeomorphism from the orbit space $|X|$ of the ep-groupoid onto the topological space $Z$, so that for any two pairs $(X, \beta)$ and $\left(X^{\prime}, \beta^{\prime}\right)$ there exists a third pair $\left(X^{\prime \prime}, \beta^{\prime \prime}\right)$ and equivalences $F:\left(X^{\prime \prime}, \beta^{\prime \prime}\right) \rightarrow(X, \beta)$ and $F^{\prime}:\left(X^{\prime \prime}, \beta^{\prime \prime}\right) \rightarrow\left(X^{\prime}, \beta^{\prime}\right)$ satisfying

$$
\beta^{\prime \prime}=\beta \circ|F|=\beta^{\prime} \circ\left|F^{\prime}\right| .
$$

Such pairs define the smooth structure on the topological space $Z$. The construction of the natural polyfold structure is carried out [15].

There are natural maps which play an important role in the GW-theory. Let us first note that $Z$ has many connected components. If $g, k \geq 0$ are integers we denote by $Z_{g, k}$ the subset of $Z$ consisting of all classes $[\alpha]=[(S, j, M, D, u)]$ of arithmetic genus $g$ and $k$ marked points. This subset is open in $Z$ and therefore is equipped with the induced polyfold structure. If $A \in H_{2}(Q, \mathbb{Z})$ is a homology class, we can also consider the open subset $Z_{A, g, k}$ consisting of elements in $Z_{g, k}$ for which the map $u$ represents $A$. Now we consider for the fixed pair $(g, k)$ the space $Z_{g, k}$. For every $i=1, \ldots, k$, we define the evaluation map at the $i$-th marked point by

$$
\mathrm{ev}_{i}: Z_{g, k} \rightarrow Q:[\alpha]=[(S, j, M, D, u)] \rightarrow u\left(m_{i}\right) .
$$

If $2 g+k \geq 3$, the forgetful map associates with $[\alpha]$ the underlying stable part of the domain $S$. It is obtained as follows.

We take a representative $(S, j, M, D, u)$ of our class $[\alpha]$ and first forget the map $u$. Now we take a component $C$ satisfying $2 g(C)+\sharp(C \cap(M \cup|D|))<3$. Since, 
by definition, our nodal surface $(S, j, M, D)$ is connected, the following cases arise. Firstly, $C$ is a sphere with one node. In this case we remove the sphere and the node. Secondly, $C$ is a sphere with two nodal pairs $\{x, y\}$ and $\left\{x^{\prime}, y^{\prime}\right\}$, where $x$ and $x^{\prime}$ lie on the sphere. In this case we remove the sphere but shortcut the two nodes by removing the two nodal pairs but adding the nodal pair $\left\{y, y^{\prime}\right\}$. Thirdly, $C$ is a sphere with one node and one marked point. In that case we remove the sphere but replace the corresponding node on the other component by the marked point. Continuing this way we end up with a stable nodal Riemann surface whose biholomorphic type does not depend on the order we weeded out the unstable components. The procedure leads to the forgetful map

$$
\sigma: Z_{g, k} \rightarrow \overline{\mathcal{M}}_{g, k}, \quad[(S, j, M, D, u)] \rightarrow\left[(S, j, M, D)_{\mathrm{stab}}\right]
$$

where $\overline{\mathcal{M}}_{g, k}$ is the standard Deligne-Mumford compactification of the moduli space of marked stable Riemann surfaces.

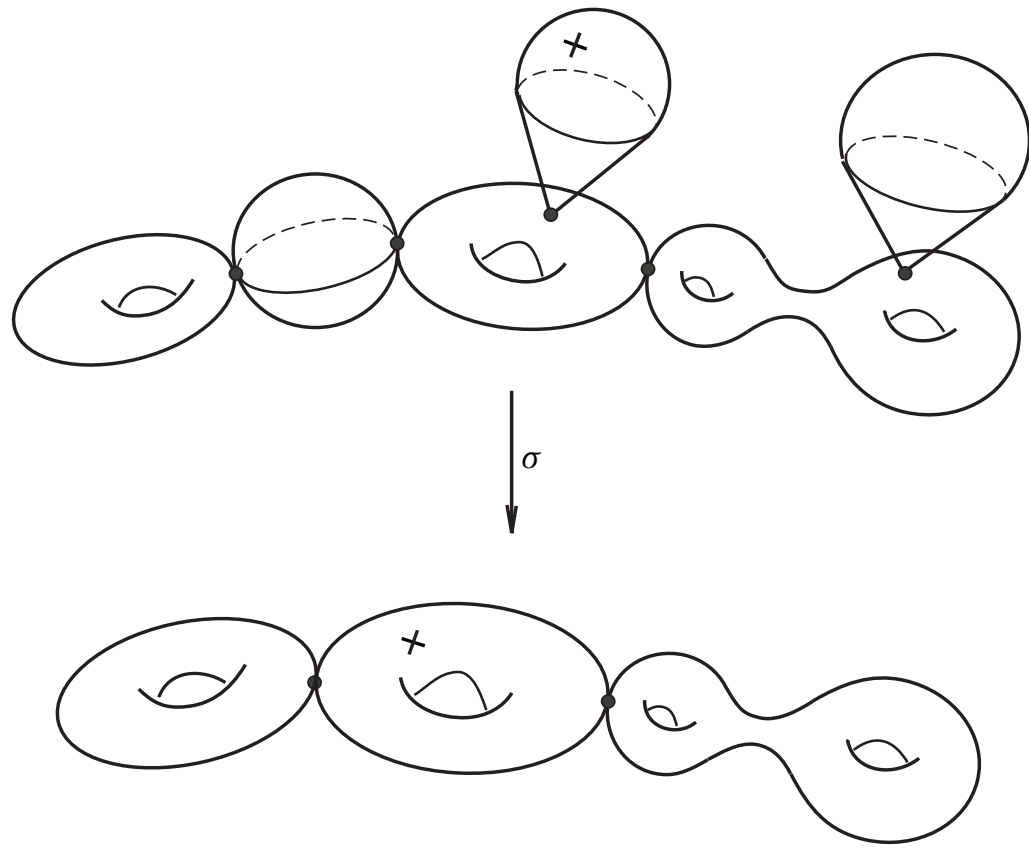

Figure 1: The forgetful map $\sigma: Z_{g, k} \rightarrow \bar{M}_{g, k}$

Theorem 1.10 The evaluation maps ev ${ }_{i}: Z_{g, k} \rightarrow Q$ and the forgetful map $\sigma: Z_{g, k} \rightarrow$ $\overline{\mathcal{M}}_{g, k}$ are sc-smooth. 
As a consequence, we can pullback the differential forms on $Q$ and on $\overline{\mathcal{M}}_{g, k}$ to obtain sc-differential forms on the polyfold $Z_{g, k}$ which can be, suitably wedged together, integrated over the smooth moduli spaces obtained from transversal Fredholm sections of strong bundles over $Z$, using the branched integration theory from [20].

Next we introduce a strong polyfold bundle $W$ over $Z$. The objects of $W$ are defined as follows. We consider multiplets

$$
\widehat{\alpha}=(S, j, M, D, u, \xi)
$$

in which the underlying stable map $\alpha=(S, j, M, D, u)$ is a representative of an element in $Z$ and $\xi$ is a continuous section along $u$ such that the mapping

$$
\xi(z): T_{z} S \rightarrow T_{u(z)} Q
$$

is complex antilinear at every $z \in S$. The domain $S$ is equipped with the complex structure $j$ and the target $Q$ is equipped with the almost complex structure $J$. We require that the map $z \mapsto \xi(z)$ is of class $H_{\mathrm{loc}}^{2}$ on $S \backslash|D|$ and it is of class $\left(2, \delta_{0}\right)$ at the points in the nodal set $|D|$. The last requirement means, taking holomorphic polar coordinates $\sigma$ around a nodal point $z$ and a smooth chart $\psi$ around its image $u(z) \in Q$, that the map

$$
(s, t) \mapsto \operatorname{pr}_{2} \circ T \psi(u(\sigma(s, t))) \xi(\sigma(s, t))\left(\frac{\partial \sigma}{\partial s}(s, t)\right)
$$

and all its partial derivatives up to order 2 , if weighted by $e^{\delta_{0}|s|}$, belong to the space $L^{2}\left([R, \infty) \times S^{1}, \mathbb{R}^{2 n}\right)$ for $R$ large enough. The definition does not depend on the choices involved.

We call two such tuples $(S, j, M, D, u, \xi)$ and $\left(S^{\prime}, j^{\prime}, M^{\prime}, D^{\prime}, u^{\prime}, \xi^{\prime}\right)$ equivalent if there exists an isomorphism

$$
\varphi:(S, j, M, D) \rightarrow\left(S^{\prime}, j^{\prime}, M^{\prime}, D^{\prime}\right)
$$

between the nodal Riemann surfaces satisfying

$$
u^{\prime} \circ \varphi=u \quad \text { and } \quad \xi^{\prime} \circ T \varphi=\xi
$$

We denote an equivalence class by $[\hat{\alpha}]=[(S, j, M, D, u, \xi)]$. The collection of all such equivalence classes constitutes the space $W$.

We have defined what it means that an element $\alpha$ represents an element on level $m$. Let us observe that if $u$ has regularity $\left(m+3, \delta_{m}\right)$, it makes sense to talk about elements $\xi$ along $u$ of regularity $\left(k+2, \delta_{k}\right)$ for $0 \leq k \leq m+1$. In the case $k=m+1$ the fiber regularity is $\left(m+3, \delta_{m+1}\right)$ and the underlying base regularity is $\left(m+3, \delta_{m}\right)$. 
The requirement of an exponential decay in the fiber towards a nodal point which is faster than the exponential decay of the underlying base curve is well-defined and independent of the charts picked to define it. Our conventions for defining the levels are governed by the overall convention that sections should be horizontal in the sense that they preserve the level structure, ie an element on level $m$ is mapped by the section to an element on bilevel $(m, m)$. Hence if the section comes from a first order differential operator we need the convention we have just used. Therefore it makes sense to say that an element

$$
\hat{\alpha}=(S, j, M, D, u, \xi)
$$

has (bi)regularity $\left(\left(m+3, \delta_{m}\right),\left(k+2, \delta_{k}\right)\right)$ as long as $k$ satisfies the above restriction $0 \leq k \leq m+1$. The equivalence class $[\widehat{\alpha}] \in W$ is said to be on level $(m, k)$ provided the pair $(u, \xi)$ has the above regularity. One of the main consequences of [15] is the following theorem in which $Z$ denotes the space of stable curves introduced in Theorem 1.9 and $W$ is the space defined above.

Theorem 1.11 (1) The set $W$ has a natural second countable paracompact topology so that the natural projection map

$$
p: W \rightarrow Z, \quad[\hat{\alpha}] \mapsto[\alpha]
$$

(forgetting the $\xi$-part) is continuous.

(2) If $Z$ is equipped with its polyfold structure, then the bundle $p$ : $W \rightarrow Z$ has the structure of a strong polyfold bundle in a natural way.

Finally, we can introduce, for a compatible smooth almost complex structure $J$ on the symplectic manifold $(Q, \omega)$, the section $\bar{\partial}_{J}$ of the strong polyfold bundle $p: W \rightarrow Z$ defined by

$$
\bar{\partial}_{J}([(S, j, M, D, u)])=\left[\left(S, j, M, D, u, \bar{\partial}_{J, j}(u)\right)\right]
$$

where $\bar{\partial}_{J, j}$ is the Cauchy-Riemann operator defined by

$$
\bar{\partial}_{J, j}(u)=\frac{1}{2}(T u+J \circ T u \circ j) .
$$

Let us call a Fredholm section of a strong polyfold bundle component-proper if the restriction to every connected component of the domain is proper. Then the following crucial result which is a special case of results proved in $[17 ; 18]$ holds true.

Theorem 1.12 The section $\bar{\partial}_{J}$ is an sc-smooth component-proper Fredholm section of the strong polyfold bundle $p: W \rightarrow Z$ having a natural orientation. 
Applying Theorem 1.3, one derives the following invariants. We abbreviate by $(\mathfrak{M}, w)=$ $\left(S\left(\bar{\partial}_{J}, \lambda\right), \lambda \bar{\partial}_{J}\right)$ the oriented and weighted solution set of the pair $\left(\bar{\partial}_{J}, \lambda\right)$ where $\lambda$ is an $\mathrm{sc}^{+}$-multisection and where the solution set is given by $S\left(\bar{\partial}_{J}, \lambda\right)=\left\{z \in Z_{g, k} \mid\right.$ $\left.\lambda\left(\bar{\partial}_{J} z\right)>0\right\}$ and the weight function $w$ is defined as $w(z)=\lambda\left(\bar{\partial}_{J} z\right)$. The set $\mathfrak{M}$ is an oriented compact branched suborbifold of $Z_{g, k}$ provided $\lambda$ is generic, ie, $\left(\bar{\partial}_{J}, \lambda\right)$ is a transversal pair.

Theorem 1.13 Let $(Q, \omega)$ be a closed symplectic manifold. Then given a homology class $A \in H_{2}(Q)$ and natural numbers $g, k \geq 0$ there exists a multilinear map

$$
\Psi_{A, g, k}^{Q}: H^{*}(Q ; \mathbb{R})^{\otimes k} \otimes H_{*}\left(\overline{\mathcal{M}}_{g, k} ; \mathbb{R}\right) \rightarrow \mathbb{R}
$$

which on $H^{*}(Q ; \mathbb{R})^{\otimes k}$ is supersymmetric with respect to the grading by even and odd forms. This map is uniquely characterized by the following formula. For a given compatible almost complex structure $J$ on $Q$ and a given small generic perturbation by an $\mathrm{sc}^{+}-$multisection $\lambda$ we have the presentation

$$
\Psi_{A, g, k}^{Q}\left(\left[\alpha_{1}\right], \ldots,\left[\alpha_{k}\right] ;[\tau]\right)=\int_{(\mathfrak{M}, w)} \operatorname{ev}_{1}^{*}\left(\alpha_{1}\right) \wedge \cdots \wedge \operatorname{ev}_{k}^{*}\left(\alpha_{k}\right) \wedge \sigma^{*}(\operatorname{PD}(\tau)) .
$$

Here PD denotes the Poincaré dual.

The a priori real number $\Psi_{A, g, k}^{Q}\left(\left[\alpha_{1}\right], \ldots,\left[\alpha_{k}\right] ;[\tau]\right)$ is called a $\mathrm{GW}$-invariant. It is zero unless the Fredholm index and the degree of the differential form which is being integrated are the same. One can show that the numbers $\Psi_{A, g, k}^{Q}\left(\left[\alpha_{1}\right], \ldots,\left[\alpha_{k}\right] ;[\tau]\right)$ are rational if the (co)homology classes are integer. The defining integral can be interpreted as a rational count of solutions of some nonlinear problem. The integration theory used above is the "branched integration" introduced in [20].

The various maps $\Psi_{A, g, k}^{Q}$ are interrelated by the so-called composition law, which also forms the basis for the Witten-Dijkgraf-Verlinde-Verlinde equation for which we refer to Manin [25], McDuff and Salamon [27] and Tian [32] and we would like to mention that the composition law follows readily from our transversality theory as will be shown in [15].

\section{Ep-groupoids and generalized maps}

In this section we shall introduce the concept of an ep-groupoid. Ep-groupoids will serve later on as models for polyfolds which is the central topic of this paper. Ep-groupoids are generalizations of proper étale Lie groupoids in which the local models for the object and morphism sets are M-polyfolds instead of finite-dimensional 
manifolds. Different ep-groupoids can serve as models for the same polyfold. This leads to the study of equivalences between ep-groupoids. We shall also introduce the concept of a generalized map and describe a construction for inverting equivalences. Our presentation of ep-groupoids follows the presentation of Lie groupoids in Moerdijk [28] and Moerdijk and Mrčun [29]. The ideas go back to A Haefliger and we refer to [10; $11 ; 12 ; 13]$.

\subsection{Ep-groupoids}

We begin by recalling the notion of a groupoid.

Definition 2.1 A groupoid $\mathfrak{G}$ is a small category whose morphisms are all invertible.

Recall that the category $\mathfrak{G}$ consists of the set of objects $G$, the set $\mathbf{G}$ of morphisms (or arrows), and the five structure maps $(s, t, i, u, m)$. Namely, the source and the target maps $s, t: \mathbf{G} \rightarrow G$ assign to every morphism, denoted by $g: x \rightarrow y$, its source $s(g)=x$ and its target $t(y)=y$, respectively. The associative multiplication (or composition) map

$$
m: \mathbf{G}_{s} \times{ }_{t} \mathbf{G} \rightarrow \mathbf{G}, \quad m(h, g)=h \circ g
$$

is defined on the fibered product

$$
\mathbf{G}_{s} \times{ }_{t} \mathbf{G}=\{(h, g) \in \mathbf{G} \times \mathbf{G} \mid s(h)=t(g)\} .
$$

For every object $x \in G$, there exists the unit morphism $1_{x}: x \rightarrow x$ in $\mathbf{G}$ which is a $2-$ sided unit for the composition, that is, $g \circ 1_{x}=g$ and $1_{x} \circ h=h$ for all morphisms $g, h \in \mathbf{G}$ satisfying $s(g)=x=t(h)$. These unit morphisms together define the unit map $u: G \rightarrow \mathbf{G}$ by $u(x)=1_{x}$. Finally, for every morphism $g: x \rightarrow y$ in $\mathbf{G}$, there exists the inverse morphism $g^{-1}: y \rightarrow x$ which is a 2-sided inverse for the composition, that is, $g \circ g^{-1}=1_{y}$ and $g^{-1} \circ g=1_{x}$. These inverses together define the inverse map $i: \mathbf{G} \rightarrow \mathbf{G}$ by $i(g)=g^{-1}$. The orbit space of a groupoid $\mathfrak{G}$,

$$
|\mathfrak{G}|=G / \sim,
$$

is the quotient of the set of objects $G$ by the equivalence relation $\sim$ defined by $x \sim y$ if and only if there exists a morphism $g: x \rightarrow y$. The equivalence class $\{y \in G \mid y \sim x\}$ will be denoted by

$$
|x|=\{y \in G \mid y \sim x\} .
$$

If $x, y \in G$ are two objects, then $\mathbf{G}(x, y)$ denotes the set of all morphisms $g: x \rightarrow y$. In particular, for $x \in G$ fixed, we denote by $\mathbf{G}(x)=\mathbf{G}(x, x)$ the stabilizer (or isotropy) group of $x$,

$$
\mathbf{G}(x)=\{\text { morphisms } g: x \rightarrow x\} \text {. }
$$


For the sake of notational economy we shall denote in the following a groupoid as well as its object set by the same letter $G$ and its morphism set by the bold letter $\mathbf{G}$.

A homomorphism $F: G \rightarrow G^{\prime}$ between two groupoids is, by definition, a functor. In particular, the two induced maps $F: G \rightarrow G^{\prime}$ and $F: \mathbf{G} \rightarrow \mathbf{G}^{\prime}$ between the object sets and the morphism sets commute with the structure maps

$$
\begin{aligned}
& s^{\prime} \circ F=F \circ s \quad t^{\prime} \circ F=F \circ t \\
& i^{\prime} \circ F=F \circ i \quad u^{\prime} \circ F=F \circ u \\
& m^{\prime} \circ(F \times F)=F \circ m \text {. }
\end{aligned}
$$

Ep-groupoids, as defined next, can be viewed as M-polyfold versions of étale and proper Lie-groupoids discussed eg in [28] and [29].

Definition 2.2 An ep-groupoid $X$ is a groupoid $X$ together with M-polyfold structures on the object set $X$ as well as on the morphism set $\mathbf{X}$ so that all the structure maps $(s, t, m, u, i)$ are sc-smooth maps and the following holds true:

- (Étale) The source and target maps $s$ and $t$ are surjective local sc-diffeomorphisms.

- (Proper) For every point $x \in X$, there exists an open neighborhood $V(x)$ so that the map $t: s^{-1}(\overline{V(x)}) \rightarrow X$ is a proper mapping.

We point out that if $X$ is a groupoid equipped with M-polyfold structures on the object set $X$ as well as on the morphism set $\mathbf{X}$, and $X$ is étale, then the fibered product $\mathbf{X}_{s} \times{ }_{t} \mathbf{X}$ has a natural $\mathbf{M}$-polyfold structure so that the multiplication map $m$ is defined on an M-polyfold. Hence its sc-smoothness is well-defined. This is proved in Lemma 2.8 below.

In an ep-groupoid every morphism $g: x \rightarrow y$ can be extended to a unique local diffeomorphism $t \circ s^{-1}$ satisfying $s(g)=x$ and $t(g)=y$. The properness assumption implies that the isotropy groups $\mathbf{G}(x)$ are finite groups.

The local structure of the morphism set of an ep-groupoid in a neighborhood of an isotropy group is described in the following theorem whose proof can be found in the Appendix, Section 5.1.

Theorem 2.3 Let $x$ be an object of an ep-groupoid $X$. Then every open neighborhood $V \subset X$ of $x$ contains an open neighborhood $U \subset V$ of $x$, a group homomorphism

$$
\varphi: \mathbf{G}(x) \rightarrow \operatorname{Diff}_{\mathrm{sc}}(U), \quad g \mapsto \varphi_{g},
$$


of the isotropy group into the group of sc-diffeomorphisms of $U$, and an sc-smooth map

$$
\Gamma: \mathbf{G}(x) \times U \rightarrow \mathbf{X}
$$

having the following properties:

- $\Gamma(g, x)=g$.

- $s(\Gamma(g, y))=y$ and $t(\Gamma(g, y))=\varphi_{g}(y)$.

- If $h: y \rightarrow z$ is a morphism between two points in $U$, then there exists a unique element $g \in \mathbf{G}(x)$ satisfying $\Gamma(g, y)=h$.

The group homomorphism $\varphi: \mathbf{G}(x) \rightarrow \operatorname{Diff}_{\mathrm{sc}}(U)$ is called a natural representation of the isotropy group $\mathbf{G}(x)$ of the element $x \in X$. The diffeomorphism $\varphi_{g}$ is given by $t \circ s^{-1}$ where $s(g)=t(g)=x$. We see that every morphism between points in $U$ belongs to the image of the map $\Gamma$ and so has an extension to precisely one of the finitely many diffeomorphisms $\varphi_{g}$ of $U$ where $g \in \mathbf{G}(x)$.

An M-polyfold $X$ is, in view of its definition in [21], equipped with a filtration

$$
X=X_{0} \supset X_{1} \supset X_{2} \supset \cdots \supset X_{\infty}:=\bigcap_{k \geq 0} X_{k}
$$

into subsets such that the injection maps $X_{k+1} \rightarrow X_{k}$ are continuous and $X_{\infty}$ is dense in all the sets $X_{k}$. Points or subsets contained in $X_{\infty}$ are called smooth points or smooth subsets. On the space $X_{k}$ there is the induced filtration $X_{k} \supset X_{k+1} \supset \cdots \supset X_{\infty}$. This M-polyfold structure of $X_{k}$ will be denoted by $X^{k}$.

If $x \in X$, we denote by $\operatorname{ml}(x) \in \mathbb{N}_{0} \cup\{\infty\}$ the largest nonnegative integer $m$ or $\infty$ so that $x \in X_{m}$. We call $m(x)$ the maximal level of $x$.

In an ep-groupoid the object set $X$ as well as the morphism set $\mathbf{X}$ are $\mathbf{M - p o l y f o l d s}$ and hence are both equipped with filtrations. Since the source and the target maps are local sc-diffeomorphisms and therefore preserve by definition the levels, we conclude that

$$
\operatorname{ml}(x)=\operatorname{ml}(y)=\operatorname{ml}(g)
$$

if $g: x \rightarrow y$ is a morphism. Consequently, the filtration of the object set $X$ induces the filtration

$$
|X|=\left|X_{0}\right| \supset\left|X_{1}\right| \supset \cdots \supset\left|X_{\infty}\right|=\bigcap_{k \geq 0}\left|X_{k}\right|
$$

of the orbit space $|X|=X / \sim$. 


\subsection{Functors and equivalences}

In this section we introduce the notion of an equivalence which plays an important role in the construction of polyfolds later on.

Definition 2.4 A functor $F: X \rightarrow Y$ between two ep-groupoids is called sc-smooth provided the induced maps between object- and morphism- spaces are sc-smooth.

In view of the functoriality of $F$, the two induced maps commute with the structure maps and therefore an sc-smooth functor $F: X \rightarrow Y$ induces an $\mathrm{sc}^{0}$-map $|F|:|X| \rightarrow|Y|$ between the orbit spaces. A continuous map is called $\mathrm{sc}^{0}$ if it preserves the natural filtrations. An important class of sc-smooth functors are the equivalences defined next.

Definition 2.5 An sc-smooth functor $F: X \rightarrow Y$ between two ep-groupoids is called an equivalence provided it has the following properties:

- The map $F$ is a local sc-diffeomorphism on objects as well as morphisms.

- The induced map $|F|:|X| \rightarrow|Y|$ between the orbit spaces is an sc-homeomorphism.

- For every $x \in X$, the map $F$ induces a bijection $\mathbf{X}(x) \rightarrow \mathbf{Y}(F(x))$ between the isotropy groups.

Clearly, if $F: X \rightarrow Y$ and $G: Y \rightarrow Z$ are equivalences, then the composition $G \circ$ $F: X \rightarrow Z$ is also an equivalence.

In general, an equivalence is not invertible as a functor. Later on, we will discuss a general procedure of inverting arrows in a category by only changing the morphism set but keeping the object set. This "inverting of arrows" for a given class of arrows is a standard procedure in category theory and we refer to Gabriel and Zisman [9].

Definition 2.6 Two sc-smooth functors $F: X \rightarrow Y$ and $G: X \rightarrow Y$ between epgroupoids $X$ and $Y$ are called naturally equivalent, if there exists an sc-smooth map $\tau: X \rightarrow \mathbf{Y}$ which associates with every object $x \in X$ an arrow $\tau(x): F(x) \rightarrow G(x)$ in $\mathbf{Y}$ and which is "natural in $x$ " in the sense that for every arrow $h: x \rightarrow x^{\prime}$ in $\mathbf{X}$ the identity

$$
\tau\left(x^{\prime}\right) \circ F(h)=G(h) \circ \tau(x)
$$


holds. The map $\tau$ is called a natural transformation.

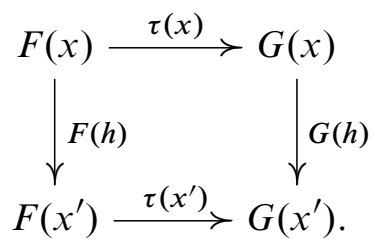

Two naturally equivalent functors $F: X \rightarrow Y$ and $G: X \rightarrow Y$ induce the same map between the orbit spaces,

$$
|F|=|G|:|X| \rightarrow|Y| .
$$

From the definitions one deduces the following result.

Proposition 2.7 Assume that the sc-smooth functors $F: X \rightarrow Y$ and $G: X \rightarrow Y$ between ep-groupoids are naturally equivalent. Then if one of the functors is an equivalence so is the other.

Proof Assume that $F: X \rightarrow Y$ is an equivalence. As remarked above the functors $F, G$ induce the same mappings between the orbit spaces so that $|F|=|G|:|X| \rightarrow|Y|$. Hence $|G|$ is a homeomorphism. If $x \in X$, we have to show that $G$ induces a bijection between the isotropy groups $\mathbf{X}(x)$ and $\mathbf{Y}(G(x))$. It suffices to show that given $g \in \mathbf{Y}(G(x))$, there exists a unique $h \in \mathbf{X}(x)$ such that $G(h)=g$. Let $\tau: X \rightarrow \mathbf{Y}$ be a natural transformation and define the morphism $f:=\tau(x)^{-1} \circ g \circ \tau(x): F(x) \rightarrow F(x)$. Since there is a bijection between $\mathbf{X}(x)$ and $\mathbf{Y}(F(x))$, there exists a unique morphism $h: x \rightarrow x$ satisfying $F(h)=f$. From $\tau(x) \circ F(h)=G(h) \circ \tau(x)$ one concludes that $g=\tau(x) \circ F(h) \circ \tau(x)^{-1}=G(h)$ which proves our claim.

Next we show that $G$ is a local sc-diffeomorphism on objects. Fix $x_{0} \in X$. Let $\mathbf{U}\left(\tau\left(x_{0}\right)\right)$ be an open neighborhood in $\mathbf{Y}$ of the morphism $\tau\left(x_{0}\right): F\left(x_{0}\right) \rightarrow G\left(x_{0}\right)$ such that the source and the target maps : $\mathbf{U}\left(\tau\left(x_{0}\right)\right) \rightarrow U\left(F\left(x_{0}\right)\right)$ and $t: \mathbf{U}\left(\tau\left(x_{0}\right)\right) \rightarrow$ $U\left(G\left(x_{0}\right)\right)$ are sc-diffeomorphisms. Since, by assumption, $F$ is a local sc-diffeomorphism on objects, there exists a an open neighborhood $U\left(x_{0}\right)$ of $x_{0}$ in $X$ such that $F: U\left(x_{0}\right) \rightarrow F\left(U\left(x_{0}\right)\right)$ is an sc-diffeomorphism. We take $U\left(x_{0}\right)$ so small that $\tau\left(U\left(x_{0}\right)\right) \subset \mathbf{U}\left(\tau\left(x_{0}\right)\right)$. Moreover, we may assume that $s: U\left(\tau\left(x_{0}\right)\right) \rightarrow F\left(U\left(x_{0}\right)\right)$ is an sc-diffeomorphism. Then $G(x)=t \circ s^{-1} \circ F(x)$ for $x \in U\left(x_{0}\right)$ and $G\left(U\left(x_{0}\right)\right)=$ $U\left(G\left(x_{0}\right)\right)$. Because $t, s^{-1}$ and $F$ are sc-diffeomorphisms also the composition $G$ is an sc- diffeomorphism on the neighborhood $U\left(x_{0}\right)$.

To complete the proof we have to show that $G: X \rightarrow Y$ is a local sc-diffeomorphism on the morphisms set. To do this we fix a morphism $g: x_{0} \rightarrow y_{0}$ between the objects 
$x_{0}$ and $y_{0}$ in $X$. By assumption, there exists an open neighborhood $\mathbf{U}(g)$ of $g$ in $\mathbf{X}$ such that $F: \mathbf{U}(g) \rightarrow F(\mathbf{U}(g))$ is an sc-diffeomorphism. Since $F$ and $G$ are local scdiffeomorphism, we find open neighborhoods $U\left(x_{0}\right)$ and $U\left(y_{0}\right)$ of the objects $x_{0}$ and $y_{0}$ such that the maps $F$ and $G$ are sc-diffeomorphism on $U\left(x_{0}\right)$ and $U\left(y_{0}\right)$. We may choose the neighborhoods $U\left(x_{0}\right), U\left(y_{0}\right)$ and $\mathbf{U}(g)$ so that the source and the target maps $s: \mathbf{U}(g) \rightarrow U\left(x_{0}\right)$ and $t: \mathbf{U}(g) \rightarrow U\left(y_{0}\right)$ are sc-diffeomorphisms. Then the set $G(\mathbf{U}(g))$ is an open subset of $\mathbf{Y}$ and the sc-smooth map $G: \mathbf{U}(g) \rightarrow G(\mathbf{U}(g))$ has an inverse given by $G^{-1}(h)=F^{-1}\left(\tau(x) \circ h \circ(\tau(y))^{-1}\right)$ for a morphism $h: G(x) \rightarrow G(y)$ belonging to $G(\mathbf{U}(g))$. Since this inverse is an sc-diffeomorphism, we have proved that also $G$ is a local sc-diffeomorphism.

Next we shall define the weak fibered product $\mathrm{L}=X \times_{Y} Z$ in which $X, Y$, and $Z$ are ep-groupoids. Consider two sc-smooth functors $F: X \rightarrow Y$ and $G: Z \rightarrow Y$ between ep-groupoids having the same target ep-groupoid $Y$. We first define the fibered product $\mathrm{L}=X \times_{Y} Z$ as a groupoid and then, under an additional assumption, as an ep-groupoid.

The object set of $\mathrm{L}$ consists of triples $(x, \varphi, z) \in X \times \mathbf{Y} \times Z$ where $\varphi: F(x) \rightarrow G(z)$ is a morphism in $\mathbf{Y}$. Hence

$$
\mathrm{L}=\{(x, \varphi, z) \in X \times \mathbf{Y} \times Z \mid s(\varphi)=F(x) \text { and } t(\varphi)=G(z)\} .
$$

In short notation, $\mathrm{L}=X_{F} \times{ }_{s} \mathbf{Y}_{t} \times{ }_{G} Z$. A morphism $l:(x, \varphi, z) \rightarrow\left(x^{\prime}, \varphi^{\prime}, z^{\prime}\right)$, between two objects in L is a triple $l=(h, \varphi, k) \in \mathbf{X} \times \mathbf{Y} \times \mathbf{Z}$ with morphisms $h: x \rightarrow x^{\prime}$ and $k: z \rightarrow z^{\prime}$ satisfying

$$
\varphi^{\prime}=G(k) \circ \varphi \circ F(h)^{-1} .
$$

Hence the set of morphisms is equal to $\mathbf{L}=\mathbf{X}_{s \circ F} \times_{s} \mathbf{Y}_{t} \times_{s \circ G} \mathbf{Z}$ or, explicitly,

$$
\mathbf{L}=\{(h, \varphi, k) \in \mathbf{X} \times \mathbf{Y} \times \mathbf{Z} \mid s \circ F(h)=s(\varphi) \text { and } t(\varphi)=s \circ G(k)\}
$$

and the source and the target maps $s, t: \mathbf{L} \rightarrow \mathrm{L}$ are defined by

$$
s(h, \varphi, k)=(s(h), \varphi, s(k)) \quad \text { and } \quad t(h, \varphi, k)=\left(t(h), \varphi^{\prime}, t(k)\right)
$$

where $\varphi^{\prime}:=G(k) \circ \varphi \circ F(h)^{-1}$. The multiplication of two morphisms is defined as

$$
(h, \varphi, k) \circ\left(h^{\prime}, \psi, k^{\prime}\right)=\left(h \circ h^{\prime}, \psi, k \circ k^{\prime}\right) .
$$

The identity morphisms $1_{(x, \varphi, z)} \in \mathbf{L}$ at the object $(x, \varphi, z) \in \mathrm{L}$ is the triple $1_{(x, \varphi, z)}=$ $\left(1_{x}, \varphi, 1_{z}\right)$. The inversion map is defined by $i(h, \varphi, k)=\left(h^{-1}, G(k) \circ \varphi \circ F(h)^{-1}, k^{-1}\right)$. With the above definitions the fibered product $\mathrm{L}=X \times_{Y} Z$ becomes a groupoid called the weak fibered product. 
In the next step we describe conditions which guarantee that $\mathrm{L}$ is an ep-groupoid. We need a lemma which is a special case of a more general result in [19].

Lemma 2.8 Let $X, Y$ and $Z$ be $M$-polyfolds. Assume that $f: X \rightarrow Y$ is a local sc-diffeomorphism and $g: Z \rightarrow Y$ is an sc-smooth map. Then the fibered product

$$
X_{f} \times{ }_{g} Z=\{(x, z) \in X \times Z \mid f(x)=g(z)\}
$$

has a natural M-polyfold structure and the projection map $\pi_{2}: X_{f} \times_{g} Z \rightarrow Z$ is a local sc-diffeomorphisms.

Proof Denote by $\triangle=\{(y, y) \mid y \in Y\}$ the diagonal in the space $Y \times Y$. Then $X_{f} \times{ }_{g} Z=(f, g)^{-1}(\triangle) \subset X \times Z$. Hence $X_{f} \times{ }_{g} Z$ is a closed subset of $X \times Z$ and consequently carries a paracompact second countable topology. Fix a point $(x, z) \in X_{f} \times g$. Since $f: X \rightarrow Y$ is a local sc-diffeomorphism, we find two open neighborhoods $U(x) \subset X$ and $V(g(z)) \subset Z$ of the points $x$ and $g(z)$ such that $f: U(x) \rightarrow V(g(z))$ is an sc-diffeomorphism. Next we find an open neighborhood $W(z) \subset Z$ of $z$ so that there exists a chart $\varphi: W \rightarrow O$ onto an open subset $O$ of a splicing core $K$ and, in addition, $g(W(z)) \subset V(g(z))$. Then $N:=(U(x) \times W(z)) \cap X_{f} \times g Z$ is an open neighborhood of $(x, z)$ in $X_{f} \times{ }_{g} Z$ and the map

$$
\Phi: N \rightarrow O, \quad(x, z) \mapsto \varphi(z)
$$

is a bijection and of class $\mathrm{sc}^{0}$. The inverse map $\Phi^{-1}: O \rightarrow N$ is equal to

$$
\left.\Phi^{-1}(k)=\left(f^{-1} \circ g \circ \varphi^{-1}(k), \varphi^{-1}(k)\right)\right)
$$

and is an $\mathrm{sc}^{0}$-map. If $\Phi_{1}: N_{1} \rightarrow O_{1}$ is defined by $(x, z) \mapsto \varphi_{1}(z)$, where $\varphi_{1}: W_{1} \rightarrow$ $O_{1} \subset K_{1}$ is another such chart map, then $\Phi_{1} \circ \Phi^{-1}: \Phi\left(N \cap N_{1}\right) \rightarrow \Phi_{1}\left(N \cap N_{1}\right)$ is equal to $\Phi_{1} \circ \Phi^{-1}(k)=\varphi_{1} \circ \varphi^{-1}(k)$ for $k \in \Phi\left(N \cap N_{1}\right)$. Hence $\Phi_{1} \circ \Phi^{-1}$ is sc-smooth. Consequently, the above charts $(\Phi, N)$ define an M-polyfold structure on $X_{f} \times{ }_{g} Z$. The construction also shows that the projection $\pi_{2}$ is a local sc-diffeomorphism.

We shall use the previous lemma in the construction of the weak fibered product of ep-groupoids.

Theorem 2.9 Let $X, Y$ and $Z$ be ep-groupoids. Assume that the functor $F: X \rightarrow Y$ is an equivalence and $G: Z \rightarrow Y$ an sc-smooth functor. Then the fibered product $X \times_{Y} Z$ has in a natural way the structure of an ep-groupoid. Moreover, the projection functor $p: X \times{ }_{Y} Z \rightarrow Z$ is an equivalence. 
Proof We apply Lemma 2.8 and view the object set of the fibered product $X \times_{Y} Z$ as

$$
X_{F} \times_{s \circ \pi_{1}}\left[\mathbf{Y}_{t} \times_{G} Z\right]
$$

Since the target map $t$ is a local sc-diffeomorphism and $G: Z \rightarrow Y$ is sc-smooth, Lemma 2.8 implies that $\mathbf{Y}_{t} \times_{G} Z$ is in a natural way an M-polyfold. The map $s \circ$ $\pi_{1}: \mathbf{Y}_{t} \times_{G} Z \rightarrow Y$ is sc-smooth and $F: X \rightarrow Y$ is a local sc-diffeomorphism. Applying Lemma 2.8 again, we conclude that the set of objects $X \times_{Y} Z$ has a natural M-polyfold structure. We could have also used instead of (2) the different bracketing

$$
\left[X_{F} \times_{S} \mathbf{Y}\right]_{t \circ \pi_{2}} \times_{G} Z
$$

which would lead to the same M-polyfold structure.

Using the fact that $s \circ F, s$, and $t$ are local sc-diffeomorphisms one also shows that the set of morphisms $\mathbf{L}=\mathbf{X}_{s \circ F} \times_{s} \mathbf{Y}_{t} \times_{s \circ G} \mathbf{Z}$ has a natural M-polyfold structure. With these $\mathbf{M}$-polyfold structures on $\mathbf{L}$ and $\mathbf{L}$, the source map

$$
s: \mathbf{L} \rightarrow \mathrm{L}, \quad s(h, \varphi, k)=(s(h), \varphi, s(k))
$$

is sc-smooth and we show that $s$ is a local diffeomorphism. Fix a morphism $(h, \varphi, k) \in$ L. Since the target map $s: \mathbf{Z} \rightarrow Z$ is a local sc-diffeomorphism, we find open neighborhoods $\mathbf{V}(k) \subset \mathbf{Z}$ of the morphism $k$ and an open neighborhood $V(s(k)) \subset Z$ of the point $s(k)$ such that $s: \mathbf{V}(k) \rightarrow V(s(k))$ is an sc-diffeomorphism. Using Lemma 2.8 and shrinking these neighborhoods if necessary, we find an open neighborhood $\mathbf{U}$ of the morphism $(h, \varphi, k)$ in $\mathbf{L}$ such that the projection $\mathbf{p}: \mathbf{U} \subset \mathbf{L} \rightarrow \mathbf{V}(k) \subset \mathbf{Z}$ is an sc-diffeomorphism. Using the second bracketing (3) and Lemma 2.8 again, we find an open neighborhood $W \subset \mathrm{L}$ of the object $(s(h), \varphi, s(k))$ such that the projection $p: \mathrm{L} \rightarrow Z$ is an sc-diffeomorphism from $W$ onto $V(s(k))$. Now observe that

$$
s\left(h^{\prime}, \varphi^{\prime}, k^{\prime}\right)=p^{-1} \circ s \circ \mathbf{p}\left(h^{\prime}, \varphi^{\prime}, k^{\prime}\right)
$$

for every $\left(h^{\prime}, \varphi^{\prime}, k^{\prime}\right) \in \mathbf{U}$. Since the right-hand side is a composition of local scdiffeomorphisms, the source map $s: \mathbf{L} \rightarrow \mathrm{L}$ on the left-hand side is a local scdiffeomorphism. Also the inversion map $i: \mathbf{L} \rightarrow \mathbf{L}$ is an sc-diffeomorphism and since the target map is the composition $t=s \circ i$ of the source map with the inverse, we conclude that the target map $t: \mathbf{L} \rightarrow \mathrm{L}$ is a local sc-diffeomorphism as well. Consequently, the multiplication map

$$
m: \mathbf{L}_{s} \times{ }_{t} \mathbf{L} \rightarrow \mathbf{L}, \quad m\left((h, \varphi, k),\left(h^{\prime}, \varphi^{\prime}, k^{\prime}\right)\right)=\left(h \circ h^{\prime}, \varphi^{\prime}, k \circ k^{\prime}\right)
$$

is well-defined and clearly sc-smooth. Next we show that $\mathrm{L}$ is proper. Pick a point $a=(x, \varphi, z) \in \mathrm{L}$. By the properness of the groupoids $X$ and $Z$, there are open 
neighborhoods $U(x)$ and $V(z)$ so that the maps

$$
t: s^{-1}(\overline{U(x)}) \rightarrow X \text { and } t: s^{-1}(\overline{V(z)}) \rightarrow Z
$$

are proper. Define the open $W(a) \subset \mathrm{L}$ by

$$
W(a)=\left\{\left(x^{\prime}, \varphi^{\prime}, z^{\prime}\right) \in L \mid x^{\prime} \in U(x), z^{\prime} \in V(z) \text { and } \varphi^{\prime}: F\left(x^{\prime}\right) \rightarrow G\left(z^{\prime}\right)\right\} .
$$

We claim that $t: s^{-1}(\overline{W(a)}) \rightarrow \mathrm{L}$ is proper. To see this, we take a sequence $\left(h_{j}, \varphi_{j}, k_{j}\right) \in$ $\mathbf{L}$ such $s\left(h_{j}, \varphi_{j}, k_{j}\right) \in \overline{W(a)}$. We may assume that after taking a subsequence $t\left(h_{j}, \varphi_{j}, k_{j}\right) \rightarrow\left(x^{\prime}, \varphi^{\prime} z^{\prime}\right)=: b$. By the definition of the target map $t: \mathbf{L} \rightarrow \mathbf{L}$, we have $t\left(h_{j}, \varphi_{j}, k_{j}\right)=\left(t\left(h_{j}\right), \varphi_{j}^{\prime}, t\left(k_{j}\right)\right)$ where $\varphi_{j}^{\prime}=G\left(k_{j}\right) \circ \varphi_{j} \circ F\left(h_{j}\right)^{-1}$. Hence the convergence of $t\left(h_{j}, \varphi_{j}, k_{j}\right)$ to $\left(x^{\prime}, \varphi^{\prime}, z^{\prime}\right)$ implies that $t\left(h_{j}\right) \rightarrow x^{\prime}, t\left(k_{j}\right) \rightarrow z^{\prime}$, and $\varphi_{j}^{\prime}=G\left(k_{j}\right) \circ \varphi_{j} \circ F\left(h_{j}\right)^{-1} \rightarrow \varphi^{\prime}$. Since $s\left(h_{j}\right) \in \overline{U(x)}$, the properness of the map $t: s^{-1}(\overline{U(x)}) \rightarrow X$ implies that after taking a subsequence, $h_{j} \rightarrow h$. The same argument shows that after taking a further subsequence we have $k_{j} \rightarrow k$. In particular, $F\left(h_{j}\right) \rightarrow F(h)$ and $G\left(k_{j}\right) \rightarrow G(k)$ and since the inversion $i$ is sc-smooth, we also have the convergence $G\left(k_{j}\right)^{-1} \rightarrow G(k)^{-1}$. From $\varphi_{j}=G\left(k_{j}\right)^{-1} \circ \varphi_{j}^{\prime} \circ F\left(h_{j}\right)$ and $\varphi_{j}^{\prime} \rightarrow \varphi^{\prime}$, we deduce that $\varphi_{j} \rightarrow G(k)^{-1} \circ \varphi^{\prime} \circ F(h)$. Hence the sequence $\left(h_{j}, \varphi_{j}, k_{j}\right)$ has a convergent subsequence which proves that $\mathrm{L}$ is proper.

Next we shall show that the projection functor

$$
p: X \times_{Y} Z \rightarrow Z
$$

is an equivalence. We already know from Lemma 2.8 that the projection $p$ is an sc-smooth functor which is a local sc-diffeomorphism on objects and morphisms. To see that $p$ induces a bijection $\mathbf{L}(a) \rightarrow \mathbf{Z}(z)$ between the isotropy groups $\mathbf{L}(a)$ and $\mathbf{Z}(z)$, we take $a=(x, \varphi, z) \in \mathrm{L}$ and $z=p(a)$. The isotropy group of $a$ is equal to

$$
\mathbf{L}(a)=\{(h, \varphi, k) \mid h: x \rightarrow x, k: z \rightarrow z, \varphi \circ F(h)=G(k) \circ \varphi\} .
$$

Given $k \in \mathbf{Z}(z)$, the morphism $\varphi^{-1} \circ G(k) \circ \varphi$ belongs to the isotropy group $\mathbf{Y}(F(x))$. Since $F$ is an equivalence, there is unique morphism $h$ belonging to the isotropy group $\mathbf{X}(x)$ such that $F(h)=\varphi^{-1} \circ G(k) \circ \varphi$. Hence the map $\mathbf{L}(a) \rightarrow \mathbf{Z}(z)$ defined by $(h, \varphi, k) \mapsto k$ is a bijection.

It remains to prove that $|p|:|\mathrm{L}| \rightarrow|Z|$ is an sc-homeomorphism. As $p$ is an sc-smooth functor, the induced map $|p|$ is of class $\operatorname{sc}^{0}$. If $|p(x, \varphi, z)|=\left|p\left(x^{\prime}, \varphi^{\prime}, z^{\prime}\right)\right|$, then $\varphi: F(x) \rightarrow G(z)$ and $\varphi: F\left(x^{\prime}\right) \rightarrow G\left(z^{\prime}\right)$. Moreover, there exists a morphism $k: z \rightarrow z^{\prime}$. Then $\left(\varphi^{\prime}\right)^{-1} \circ G(k) \circ \varphi$ is a morphism between $F(x)$ and $F\left(x^{\prime}\right)$. Because $|F|:|X| \rightarrow$ $|Y|$ is a bijection, there exists a unique morphism $h: x \rightarrow x^{\prime}$ such that $F(h)=$ $\left(\varphi^{\prime}\right)^{-1} \circ G(k) \circ \varphi$. This implies that $(h, \varphi, k) \in \mathbf{L}$ is a morphism between $(x, \varphi, z)$ and $\left(x^{\prime}, \varphi^{\prime}, z^{\prime}\right)$ showing that both of these triples belong to the same equivalence class 
in the orbit space $|L|$. So, $|p|$ is an injection. If $|z| \in|Z|$, then $|F||x|=|G||z|$ for some $|x| \in|X|$, that is, $|G(z)|=|F(x)|$. This means that there is a morphism $\varphi: F(x) \rightarrow G(z)$ so that the morphism $\varphi^{-1} \circ 1_{G(z)} \circ \varphi$ belongs to the isotropy group $\mathbf{Y}(F(x))$. Hence there exists an element $h \in \mathbf{X}(x)$ so that $F(h)=\varphi^{-1} \circ 1_{G(z)} \circ \varphi$. This implies that $(x, h, y) \in \mathrm{L}$ and $|p||(x, h, z)|=|z|$ proving that $|p|$ is a surjection. Consider $|a| \in|\mathrm{L}|$ with the representative $a=(x, \varphi, z) \in L$. Since $p: \mathrm{L} \rightarrow Z$ is a local sc-diffeomorphism, there exists an open neighborhood $U(a)$ of the point $a$ in $\mathrm{L}$ and an open neighborhood $V(z)$ of the point $z$ in $Z$ so that $p: U(a) \rightarrow V(z)$ is an scdiffeomorphism. The quotient maps $\pi_{1}: \mathrm{L} \rightarrow|\mathrm{L}|$ and $\pi_{2}: Z \rightarrow|Z|$ are open. Hence $|U(a)|$ and $|V(z)|$ are open neighborhoods of the equivalence class $[a]$ and $[z]$ in $|\mathrm{L}|$ and $|Z|$, respectively. From $|p|(|U(a)|)=\pi_{2} \circ p \circ \pi_{1}(|U(a)|)=|V(z)|$ it follows that $|p|$ maps open sets in $|\mathrm{L}|$ onto open sets in $|Z|$. Therefore, $|p|^{-1}=\left|p^{-1}\right|:|Z| \rightarrow|\mathrm{L}|$ is continuous. Because also $|p|:|\mathrm{L}| \rightarrow|Z|$ is continuous and a bijection, the map $|p|$ is a homeomorphism. This finishes the proof of Theorem 2.9.

The next result is important for our constructions later on.

Proposition 2.10 Assume that the functors $F: X \rightarrow Y$ and $G: Z \rightarrow Y$ between epgroupoids are equivalences. Then there exists a third ep-groupoid $\mathrm{L}$ and equivalences

$$
\Phi: \mathrm{L} \rightarrow X \text { and } \Psi: \mathrm{L} \rightarrow Z
$$

so that the compositions $F \circ \Phi$ and $G \circ \Psi: \mathrm{L} \rightarrow Y$ are naturally equivalent.

Proof Set $\mathrm{L}=X \times{ }_{Y} Z$. Since $F$ and $G$ are equivalences and therefore local sc-diffeomorphisms, Theorem 2.9 implies that the projections

$$
\pi_{1}: \mathrm{L} \rightarrow X \text { and } \pi_{2}: \mathrm{L} \rightarrow Z
$$

are equivalences. A natural transformation between $F \circ \pi_{1}$ and $G \circ \pi_{2}$ is given by the sc-smooth map $\tau$ defined by $\tau(x, \varphi, z)=\varphi$.

\subsection{Inversion of equivalences and generalized maps}

As we shall see later on the ep-groupoids can be viewed as models for polyfolds. If two ep-groupoids have an equivalence between them they will turn out to be models for the same polyfold. In this subsection we introduce the notion of a generalized map between ep-groupoids. Later on generalized maps will descend to maps between polyfolds.

So far we have constructed a category whose objects are the polyfold groupoids and whose morphisms between them are the sc-smooth functors. There is a distinguished 
family of morphisms, namely the equivalences. Equivalences are usually not invertible (as functors). However, there is a category-theoretic procedure for inverting a class of prescribed arrows in a given category while at the same time keeping the objects and only minimally changing the morphisms. The general procedure is described in Gabriel and Zisman [9]. In [28], Moerdijk describes the procedure in the case of Lie groupoids, which are used to give a definition of an orbifold. Modulo the modifications necessitated by the fact that we work in the splicing world we follow Moerdijk's description. We define a new category whose objects are the ep-groupoids and whose morphisms " $X \Longrightarrow Y$ " are equivalence classes of diagrams of the form

$$
X \stackrel{F}{\leftarrow} A \stackrel{\Phi}{\rightarrow} Y
$$

where $X, Y$, and $A$ are ep-groupoids and where $F$ is an equivalence and $\Phi$ is an sc-smooth functor. A second such diagram

$$
X \stackrel{F^{\prime}}{\longleftarrow} A^{\prime} \stackrel{\Phi^{\prime}}{\longrightarrow} Y
$$

is called a refinement of the first diagram, if there exists an equivalence $H: A^{\prime} \rightarrow A$ so that the functors $F \circ H$ and $F^{\prime}: A^{\prime} \rightarrow X$ are naturally equivalent as well as $\Phi \circ H$ and $\Phi^{\prime}: A^{\prime} \rightarrow Y$ as illustrated in the diagram below. It is clear that given three diagrams which connect $X$ with $Y$ so that $d^{\prime}$ refines $d$ and $d^{\prime \prime}$ refines $d^{\prime}$, then $d^{\prime \prime}$ refines $d$.

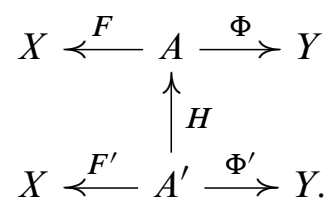

Definition 2.11 Two diagrams $X \stackrel{F}{\longleftarrow} A \stackrel{\Phi}{\rightarrow} Y$ and $X \stackrel{F^{\prime}}{\longleftarrow} A^{\prime} \stackrel{\Phi^{\prime}}{\longrightarrow} Y$ as above are called equivalent if they have a common refinement.

The notion of having a common refinement is clearly reflexive and symmetric on diagrams of the above form. Let us show that it is also transitive, so that it indeed defines an equivalence relation.

Proposition 2.12 Assume that $d, d^{\prime}$ and $d^{\prime \prime}$ are three diagrams connecting the epgroupoids $X$ with $Y$ and assume that the diagrams $\left(d, d^{\prime}\right)$ and $\left(d^{\prime}, d^{\prime \prime}\right)$ have common refinements. Then also the two diagrams $d, d^{\prime \prime}$ have a common refinement.

Proof Assume that $b$ and $b^{\prime}$ are the common refinements of the diagrams $\left(d, d^{\prime}\right)$ and $\left(d^{\prime}, d^{\prime \prime}\right)$, respectively. In particular, the diagrams $b$ and $b^{\prime}$ are common refinements of 
the diagram $d^{\prime}$. In view of the remarks proceeding the proposition, it suffices to prove that $b$ and $b^{\prime}$ have a common refinement. To prove this, assume that the diagrams

$$
b: X \stackrel{G}{\longleftarrow} B \stackrel{\Psi}{\rightarrow} Y \quad \text { and } \quad b^{\prime}: X \stackrel{G^{\prime}}{\longleftarrow} B^{\prime} \stackrel{\Psi^{\prime}}{\longrightarrow} Y
$$

are refinements of the diagram $X \stackrel{F}{\leftarrow} A \stackrel{\Phi}{\rightarrow} Y$. This situation is illustrated by the two diagrams
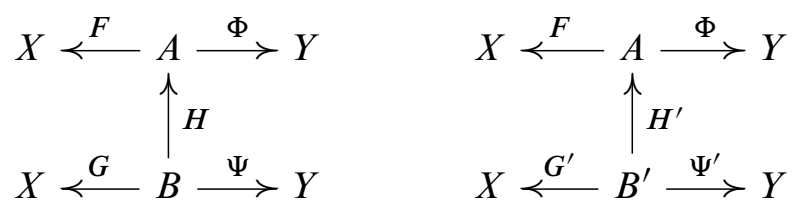

where the functors $H: B \rightarrow A$ and $H^{\prime}: B^{\prime} \rightarrow A$ are equivalences. We take the fibered product $\mathrm{L}=B \times_{A} B^{\prime}$ and define the diagram $c$ by

$$
c: X \stackrel{G \circ \pi_{1}}{\longleftarrow} \mathrm{L} \stackrel{\Psi \circ \pi_{1}}{\longrightarrow} Y .
$$

We claim that $c$ is a common refinement of the diagrams $b$ and $b^{\prime}$. In view of Theorem 2.9 , the projections $\pi_{1}: \mathrm{L} \rightarrow B$ and $\pi_{2}: \mathrm{L} \rightarrow B^{\prime}$ are equivalences. We have to show that $\Psi \circ \pi_{1}$ and $\Psi^{\prime} \circ \pi_{2}: \mathrm{L} \rightarrow Y$ are naturally equivalent as well as $G \circ \pi_{1}$ and $G^{\prime} \circ \pi_{2}: \mathrm{L} \rightarrow X$. Note that if $\left(x, \varphi, x^{\prime}\right) \in \mathrm{L}$, then

$$
\Psi^{\prime} \circ \pi_{2}\left(x, \varphi, x^{\prime}\right)=\Psi^{\prime}\left(x^{\prime}\right), \quad \Psi \circ \pi_{1}\left(x, \varphi, x^{\prime}\right)=\Psi(x) .
$$

and $\varphi: H(x) \rightarrow H^{\prime}\left(x^{\prime}\right)$. Since the functors $\Phi \circ H$ and $\Psi$ as well as $\Phi \circ H^{\prime}$ and $\Psi^{\prime}$ are naturally equivalent, there exist two sc-smooth maps $\tau_{1}: B \rightarrow \mathbf{Y}$ and $\tau_{2}: B^{\prime} \rightarrow \mathbf{Y}$ such that $\tau_{1}(x)$ is a morphism $\Phi \circ H(x) \rightarrow \Psi(x)$ and $\tau_{2}\left(x^{\prime}\right)$ is a morphism $\Phi \circ H^{\prime}\left(x^{\prime}\right) \rightarrow$ $\Psi^{\prime}\left(x^{\prime}\right)$. The sc-smooth map $\tau: \mathrm{L} \rightarrow \mathbf{Y}$, given by

$$
\tau\left(x, \varphi, x^{\prime}\right)=\tau_{2}\left(x^{\prime}\right) \circ \Phi(\varphi) \circ \tau_{1}(x)^{-1},
$$

defines the natural equivalence between the functors $\Psi^{\prime} \circ \pi_{2}$ and $\Psi \circ \pi_{1}: \mathrm{L} \rightarrow Y$. Similar arguments prove that the two functors $G \circ \pi_{1}$ and $G^{\prime} \circ \pi_{2}: \mathrm{L} \rightarrow X$ are naturally equivalent. This completes the proof of the proposition.

Definition 2.13 Let $X$ and $Y$ be ep-groupoids. A generalized map $\mathfrak{a}: X \Rightarrow Y$ is by definition the equivalence class of a diagram

$$
d: X \stackrel{F}{\leftarrow} A \stackrel{\Phi}{\rightarrow} Y
$$

where $A$ is an ep-groupoid and where $F$ is an equivalence and $\Phi$ is an sc-smooth functor. We shall use the notation

$$
\mathfrak{a}=[d]=[X \stackrel{F}{\longleftarrow} A \stackrel{\Phi}{\rightarrow} Y] .
$$


The composition $\mathfrak{b} \circ \mathfrak{a}$ of the generalized maps $\mathfrak{a}: X \Rightarrow Y$ and $\mathfrak{b}: Y \Rightarrow Z$ is defined as follows. We choose representatives

$$
X \stackrel{F}{\leftarrow} A \stackrel{\Phi}{\rightarrow} Y \text { and } Y \stackrel{G}{\leftarrow} B \stackrel{\Psi}{\rightarrow} Z
$$

of the equivalence classes $\mathfrak{a}$ and $\mathfrak{b}$, and consider the diagram

$$
X \stackrel{F}{\leftarrow} A \stackrel{\Phi}{\rightarrow} Y \stackrel{G}{\leftarrow} B \stackrel{\Psi}{\rightarrow} Z .
$$

We replace the middle portion $A \stackrel{\Phi}{\rightarrow} Y \stackrel{G}{\leftarrow} B$ of the diagram by the ep-groupoid $A \times_{Y} B$. In view of Theorem 2.9, the projection $\pi_{1}: A \times_{Y} B \rightarrow A$ is an equivalence and we can build the diagram

$$
X \stackrel{F \circ \pi_{1}}{\longleftarrow} A \times_{Y} B \stackrel{\Psi \circ \pi_{2}}{\longrightarrow} Z .
$$

The map $F \circ \pi_{1}$ is an equivalence and the map $\Psi \circ \pi_{2}$ an sc-smooth functor. If

$$
X \stackrel{F^{\prime}}{\longleftarrow} A^{\prime} \stackrel{\Phi^{\prime}}{\longrightarrow} Y
$$

is equivalent to the diagram $d$ and

$$
Y \stackrel{G^{\prime}}{\longleftarrow} B^{\prime} \stackrel{\Psi^{\prime}}{\longrightarrow} Z
$$

is equivalent to the diagram $d_{1}$, then the two diagrams

$$
X \stackrel{F \circ \pi_{1}}{\longleftarrow} A \times_{Y} B \stackrel{\Psi \circ \pi_{2}}{\longrightarrow} Z \text { and } X \stackrel{F^{\prime} \circ \pi_{1}}{\longleftarrow} A^{\prime} \times_{Y} B^{\prime} \stackrel{\Psi^{\prime} \circ \pi_{2}}{\longrightarrow} Z
$$

are equivalent. Therefore, the composition of the equivalence classes having the representatives

$$
X \stackrel{F}{\leftarrow} A \stackrel{\Phi}{\rightarrow} Y \quad \text { and } \quad Y \stackrel{G}{\leftarrow} B \stackrel{\Psi}{\rightarrow} Z
$$

can be defined as the equivalence class of the diagram (4). The composition of the equivalence classes is associative. The identity morphism $1_{X}$ of the groupoid $X$ can be identified with the equivalence class of the diagram

$$
X \stackrel{1_{X}}{\longleftarrow} X \stackrel{1_{X}}{\longrightarrow} X .
$$

The compositions of the generalized map a: $X \Rightarrow Y$ with the equivalence classes of the diagrams

$$
X \stackrel{1_{X}}{\longleftarrow} X \stackrel{1_{X}}{\longrightarrow} X \quad \text { and } Y \stackrel{1_{Y}}{\longleftarrow} Y \stackrel{1_{Y}}{\longrightarrow} Y
$$

give the generalized map a. Consequently, we have constructed the category whose objects are ep-groupoids and whose morphisms are generalized maps. We denote this category by $\mathfrak{E p}$. 
If the functor $F: X \rightarrow Y$ is an equivalence, then the diagram $Y \stackrel{F}{\leftarrow} X \stackrel{F}{\rightarrow} Y$ is a refinement of (5) and hence belongs to the class $1_{Y}$. A smooth functor $\Phi: X \rightarrow Y$ can be identified with the equivalence class of the diagram

$$
X \stackrel{1_{X}}{\longleftarrow} X \stackrel{\Phi}{\rightarrow} Y
$$

denoted by $[\Phi]$. As a special case, an equivalence functor $F: X \rightarrow Y$ is identified with the equivalence class of the diagram

$$
X \stackrel{1_{X}}{\longleftarrow} X \stackrel{F}{\rightarrow} Y .
$$

This diagram defines the equivalence class $[F]$. Its inverse, denoted by $[F]^{-1}$, is readily identified with the equivalence class of the diagram

$$
Y \stackrel{F}{\leftarrow} X \stackrel{\operatorname{id}_{X}}{\longrightarrow} X
$$

so that $[F] \circ[F]^{-1}=1_{Y}$ and $[F]^{-1} \circ[F]=1_{X}$.

We see that those functors which originally are equivalences become invertible in the new category.

The following lemma explains the relationship between generalized maps and induced maps between the underlying orbit space. The proof is straightforward.

Lemma 2.14 Let $d: X \stackrel{F}{\leftarrow} A \stackrel{\Phi}{\rightarrow} Y$ be a diagram between ep-groupoids in which $F$ is an equivalence and $\Phi$ an sc-smooth functor. Then $d$ induces an $\mathrm{sc}^{0}$-map $|d|:|X| \rightarrow|Y|$ between the orbit spaces defined by

$$
|d|=|\Phi| \circ|F|^{-1} .
$$

If a second diagram $d^{\prime}$ refines $d$, then $\left|d^{\prime}\right|=|d|$. In particular, equivalent diagrams induce the same map $|X| \rightarrow|Y|$ between orbit spaces.

Hence a generalized map $X \Rightarrow Y$ between ep-groupoids which is an equivalence class $[d]$ of diagrams induces the canonical map $|[d]|:=|d|:|X| \rightarrow|Y|$ between the orbit spaces.

One might ask if one can characterize invertible generalized maps, ie, generalized maps $\mathfrak{a}$ which have an inverse in $\mathfrak{E} \mathfrak{p}$. An obvious conjecture is that an invertible generalized map a: $X \Longrightarrow Y$ can be represented by a diagram

$$
X \stackrel{F}{\leftarrow} A \stackrel{G}{\rightarrow} Y
$$


in which $F$ and $G$ are equivalences. This is, in fact, true if one deals with the special case of étale proper Lie groupoids (of finite dimension). The standard proof of the above fact uses the inverse function theorem and a dimension argument and this part cannot be generalized. In fact, it seems doubtful to be true in our more general framework. Therefore, we introduce the following two notions.

Definition 2.15 A generalized map a: $X \Rightarrow Y$ is called invertible if there exists a generalized map $\mathfrak{b}: Y \Longrightarrow X$ satisfying $\mathfrak{b} \circ \mathfrak{a}=1_{X}$ and $\mathfrak{a} \circ \mathfrak{b}=1_{Y}$. We call a generalized map a strongly invertible, or s-invertible for short, if it is the equivalence class of a diagram

$$
X \stackrel{F}{\leftarrow} A \stackrel{G}{\rightarrow} Y
$$

in which both functors $F$ and $G$ are equivalences. An s-invertible element is called an s-isomorphism.

If $\mathfrak{a}=[X \stackrel{F}{\longleftarrow} A \stackrel{G}{\rightarrow} Y]$ is s-invertible, then its inverse $\mathfrak{a}^{-1}$ can be represented by the diagram

$$
Y \stackrel{G}{\longleftarrow} A \stackrel{F}{\rightarrow} X .
$$

Indeed, the composition

$$
[Y \stackrel{G}{\leftarrow} A \stackrel{F}{\rightarrow} X] \circ[X \stackrel{F}{\leftarrow} A \stackrel{G}{\rightarrow} Y]
$$

is represented by the diagram

$$
X \stackrel{F \circ \pi_{1}}{\longleftarrow} A \times_{Y} A \stackrel{F \circ \pi_{2}}{\longrightarrow} X
$$

which is refined by the diagram $X \stackrel{F}{\leftarrow} A \stackrel{F}{\rightarrow} X$ in view of the equivalence $A \rightarrow A \times_{Y} A$ defined as $a \rightarrow\left(a, 1_{G(a)}, a\right)$. The diagram

$$
X \stackrel{F}{\leftarrow} A \stackrel{F}{\rightarrow} X
$$

also refines the identity diagram

$$
X \stackrel{1_{X}}{\longleftarrow} X \stackrel{1_{X}}{\longrightarrow} X
$$

via the equivalence $F: A \rightarrow X$. Hence our candidate for $\mathfrak{a}^{-1}$ is indeed the inverse of $\mathfrak{a}$.

This shows that the inverse of an s-invertible element is s-invertible. It is easily verified that the composition of two s-invertible maps is s-invertible. 


\subsection{Strong bundles over ep-groupoids}

In this section we introduce the notion of a strong bundle over ep-groupoid and extends the previously developed ideas of equivalences and generalized maps to this context.

We consider an ep-groupoid $X=(X, \mathbf{X})$ and a strong M-polyfold bundle

$$
p: E \rightarrow X
$$

over the object space $X$ of the ep-groupoid. Strong M-polyfold bundles are defined in Definition 4.9 of [21]. In particular, $E$ is an M-polyfold of type 1 and $X$ is an $\mathrm{M}$-polyfold of type 0 . Moreover, $p: E \rightarrow X$ is a surjective sc-smooth map and the fibers

$$
p^{-1}(x)=E_{x}
$$

over $x \in X$ carry the structure of a Banach space. Since the source map $s: \mathbf{X} \rightarrow X$ is by definition a local sc-diffeomorphism, the fibered product

$$
\mathbf{X}_{s} \times{ }_{p} E=\{(g, e) \in \mathbf{X} \times E \mid s(g)=p(e)\}
$$

is an M-polyfold in view of Lemma 2.8. Moreover, the bundle

$$
\mathbf{E}=\mathbf{X}_{s} \times_{p} E \stackrel{\pi_{1}}{\longrightarrow} \mathbf{X}
$$

is, as the pullback of a strong M-polyfold bundle, also a strong M-polyfold bundle in view of Proposition 4.11 in [21].

Now we assume that there exists a strong bundle map $\mu: \mathbf{E} \rightarrow E$ which covers the target map $t: \mathbf{X} \rightarrow X$ of the ep-groupoid so that

$$
t \circ \pi_{1}(g, e)=p \circ \mu(g, e)
$$

for all $(g, e) \in \mathbf{X}_{s} \times_{p} E$ :

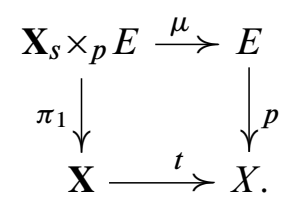

In addition, we assume that the postulated bundle map $\mu$ satisfies the following properties:

- $\mu$ is a surjective local sc-diffeomorphism and linear on the fibers $E_{x}$.

- $\mu\left(1_{x}, e_{x}\right)=e_{x}$ for all $x \in X$ and $e_{x} \in E_{x}$.

- $\mu(g \circ h, e)=\mu(g, \mu(h, e))$ for all $g, h \in \mathbf{X}$ and $e \in E$ satisfying $s(h)=p(e)$ and $t(h)=s(g)=p(\mu(h, e))$. 
It follows, in particular, that

$$
\mu(g, \cdot): E_{x} \rightarrow E_{y}
$$

is a linear isomorphism if $g: x \rightarrow y$ is a morphism in $\mathbf{X}$.

With the above data we shall define the ep-groupoid

$$
E=(E, \mathbf{E})
$$

in which the object set $E$ is the M-polyfold $E$ we started with and the morphism set $\mathbf{E}$ is the above fibered product $\mathbf{X}_{s} \times_{p} E$. The source and target maps $s, t: \mathbf{E} \rightarrow E$ are defined as follows:

$$
\begin{aligned}
& s(g, e)=e \\
& t(g, e)=\mu(g, e)
\end{aligned}
$$

for $(g, e) \in \mathbf{X}_{s} \times{ }_{p} E$. These maps $s$ and $t$ are fiberwise linear surjective local $\mathrm{sc}_{\triangleleft}-$ diffeomorphisms covering the source and target maps $\mathbf{X} \rightarrow X$. Indeed,

$$
\begin{aligned}
& p \circ s(g, e)=p(e)=s(g) \\
& p \circ t(g, e)=p \circ \mu(g, e)=t \circ \pi_{1}(g, e)=t(g) .
\end{aligned}
$$

The identity morphism at $e_{x} \in E_{x}$ is the pair $\left(1_{x}, e_{x}\right) \in \mathbf{X}$ and if $g: x \rightarrow y$ is a morphism in $\mathbf{X}$, the inverse of $\left(g, e_{x}\right) \in \mathbf{E}$ is the pair $i\left(g, e_{x}\right)=\left(g^{-1}, \mu\left(g, e_{x}\right)\right)$. The multiplication map in $E$ is defined by

$$
(h, f) \circ(g, e):=(h \circ g, e)
$$

whenever $f=\mu(g, e) \in E$.

The two sc-smooth projection maps $p: E \rightarrow X$ and $\pi_{1}: \mathbf{E} \rightarrow \mathbf{X}$ together define an sc-smooth functor denoted by

$$
P: E \rightarrow X
$$

between the two ep-groupoids $E=(E, \mathbf{E})$ and $X=(X, \mathbf{X})$.

We shall refer to this functor $P: E \rightarrow X$ as to a strong bundle over the ep-groupoid $X$.

Such a strong bundle is, in particular, an ep-groupoid together with a functor $P$ onto the base ep-groupoid and we shall use the same letter for the two induced maps on the object and the morphism sets, namely,

$$
E \stackrel{P}{\rightarrow} X \quad \text { and } \quad \mathbf{E} \stackrel{P}{\rightarrow} \mathbf{X} .
$$


Definition 2.16 A linear strong bundle morphism $\Phi: P \rightarrow P^{\prime}$ between the two strong bundles $P: E \rightarrow X$ and $P^{\prime}: E^{\prime} \rightarrow X^{\prime}$ over the ep-groupoids $X$ and $X^{\prime}$ consists of a functor $\Phi: E=(E, \mathbf{E}) \rightarrow E^{\prime}=\left(E^{\prime}, \mathbf{E}^{\prime}\right)$ between ep-groupoids which is linear on the fibers and which covers an sc-functor $\varphi: X \rightarrow X^{\prime}$ between the bases. Moreover, the functor $\Phi$ induces strong bundle maps $\Phi: E \rightarrow E^{\prime}$ and $\Phi: \mathbf{E} \rightarrow \mathbf{E}^{\prime}$ between the object sets and morphism sets:

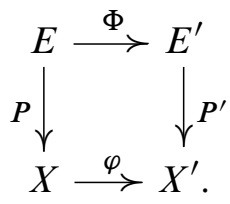

There is a distinguished class of linear strong bundle morphisms which generalizes equivalences between ep-groupoids.

Definition 2.17 A linear strong bundle equivalence $\Phi: P \rightarrow P^{\prime}$ between the two strong bundles $P: E \rightarrow X$ and $P^{\prime}: E^{\prime} \rightarrow X^{\prime}$ over ep-groupoids is a linear strong bundle morphism $\Phi: P \rightarrow P^{\prime}$ satisfying the following properties:

(1) The functor $\Phi: E \rightarrow E^{\prime}$ is an equivalence of ep-groupoids, covering the equivalence $\varphi: X \rightarrow X^{\prime}$ between the underlying ep-groupoids.

(2) The induced maps $\Phi: E \rightarrow E^{\prime}$ and $\Phi: \mathbf{E} \rightarrow \mathbf{E}^{\prime}$ between the object sets and the morphism sets preserve the strong bundle structures and are locally strong bundle isomorphisms.

For notational convenience we shall abbreviate these notions as follows:

$$
\begin{array}{ll}
\text { bundle map } & :=\text { linear strong bundle morphism; } \\
\text { bundle equivalence } & :=\text { linear strong bundle equivalence. }
\end{array}
$$

To generalize the notion of natural equivalence, we consider two strong bundles $P: E \rightarrow$ $X$ and $P^{\prime}: E^{\prime} \rightarrow X^{\prime}$ over ep-groupoids and two bundle maps

$$
\Phi, \Psi: P \rightarrow P^{\prime} .
$$

Definition 2.18 The bundle maps $\Phi$ and $\Psi$ are called naturally equivalent, if there exists a natural transformation

$$
T: E \rightarrow \mathbf{E}^{\prime}
$$

for the two functors $\Phi, \Psi: E \rightarrow E^{\prime}$ between ep-groupoids (in the sense of Definition 2.6) and a natural transformation

$$
\tau: X \rightarrow \mathbf{X}^{\prime}
$$


between the underlying functors $\varphi, \psi: X \rightarrow X^{\prime}$ which commute with the functors $P: E \rightarrow X$ and $P^{\prime}: E^{\prime} \rightarrow X^{\prime}$ so that

$$
\tau \circ P(e)=P^{\prime} \circ T(e), \quad e \in E .
$$

To reformulate the above definition we derive a simple description of the natural transformation $T: \Phi \rightarrow \Psi$ as a canonical lift of the underlying natural transformation $\tau: \varphi \rightarrow \psi$. By definition, the map

$$
T: E \rightarrow \mathbf{E}^{\prime}:=\mathbf{X}_{s}^{\prime} \times{ }_{P^{\prime}} E^{\prime},
$$

which covers the natural transformation $\tau: X \rightarrow \mathbf{X}^{\prime}$ has the form

$$
T(e)=(\tau(P(e)), A(e)) .
$$

Since $\Phi(e)=s(T(e))=A(e)$ and $\Psi(e)=t(T(e))=\mu(\tau(P(e)), A(e))$, it follows that

$$
T(e)=(\tau(P(e)), \Phi(e)) \quad \text { and } \quad \Psi(e)=\mu(\tau(P(e)), \Phi(e)) .
$$

This implies that $T$ is a strong bundle map between the bundles $E \rightarrow X$ and $\mathbf{X}_{s}^{\prime} \times{ }_{P^{\prime}} E^{\prime} \rightarrow \mathbf{X}^{\prime}$ covering $\tau$. In view of this discussion we obtain an equivalent definition as follows.

Definition 2.19 A natural transformation $T$ between bundle maps $\Phi: P \rightarrow P^{\prime}$ and $\Psi: P \rightarrow P^{\prime}$ (covering the underlying functors $\varphi: X \rightarrow X^{\prime}$ and $\psi: X \rightarrow X^{\prime}$ ) is a strong bundle map

$$
T: E \rightarrow \mathbf{X}^{\prime} \times{ }_{P^{\prime}} E^{\prime}
$$

covering a natural transformation $\tau: X \rightarrow \mathbf{X}^{\prime}$ between the functors $\varphi$ and $\psi$ of the form

$$
T(e)=(\tau(P(e)), \Phi(e))
$$

and satisfying

$$
\mu(\tau(P(e)), \Phi(e))=\Psi(e) .
$$

Let us denote by $\mathfrak{S} \mathfrak{E p}$ the category whose objects are strong bundles over ep-groupoids. As in the case of the category $\mathfrak{E} \mathfrak{p}$ we take as morphisms a class of generalized maps introduced next. We consider two strong bundles $P: E \rightarrow X$ and $P^{\prime}: E^{\prime} \rightarrow X^{\prime}$ over ep-groupoids and study the diagrams

$$
E \stackrel{\Phi}{\leftarrow} E^{\prime \prime} \stackrel{\Psi}{\rightarrow} E^{\prime},
$$

where $P^{\prime \prime}: E^{\prime \prime} \rightarrow X^{\prime \prime}$ is a third strong bundle over an ep-groupoid, and where $\Phi$ is a bundle equivalence and $\Psi$ a bundle map. Following the earlier construction of a generalized map between ep-groupoids, one introduces the notion of a refinement of a 
diagram and then calls two diagrams equivalent, if they possess a common refinement. The equivalence class of the diagram

$$
E \stackrel{\Phi}{\leftarrow} E^{\prime \prime} \stackrel{\Psi}{\rightarrow} E^{\prime},
$$

is then, by definition, a generalized strong bundle map, for simplicity called a generalized bundle map and denoted by

$$
\text { A: } E \Rightarrow E^{\prime} \text {. }
$$

By

$$
\mathfrak{a}: X \Rightarrow X^{\prime}
$$

we shall denote the associated underlying generalized map between the base epgroupoids. One has to keep in mind that in the fibers the induced maps are linear and preserve the strong bundle structure. The notion of invertible and s-invertible are defined as before (Definition 2.15), namely as follows.

Definition 2.20 A generalized bundle map $\mathfrak{A}: E \Rightarrow E^{\prime}$ is called invertible if there exists a generalized bundle map $\mathfrak{B}: E^{\prime} \Rightarrow E$ satisfying $\mathfrak{B} \circ \mathfrak{A}=1_{E}$ and $\mathfrak{A} \circ \mathfrak{B}=1_{E^{\prime}}$. The generalized bundle map $\mathfrak{A}: E \Rightarrow E^{\prime}$ is called s-invertible, if it can be represented by a diagram

$$
E \stackrel{\Phi}{\longleftarrow} E^{\prime \prime} \stackrel{\Psi}{\rightarrow} E^{\prime}
$$

in which both bundle maps are equivalences.

An s-invertible generalized bundle map $\mathfrak{A}: E \Rightarrow E^{\prime}$ will in the following be called an s-bundle isomorphism.

We point out that an s-bundle isomorphism $\mathfrak{A}: E \Rightarrow E^{\prime}$ covers automatically an s-isomorphism a: $X \Rightarrow X^{\prime}$ between the underlying base ep-groupoids.

Definition 2.21 The two strong bundles $P: E \rightarrow X$ and $P^{\prime}: E^{\prime} \rightarrow X^{\prime}$ over epgroupoids are called strong bundle equivalent, if there exists an s-bundle isomorphism $\mathfrak{A}: E \Rightarrow E^{\prime}$.

Definition 2.22 A (sc-smooth) section of the strong bundle $P: E \rightarrow X$ over the ep-groupoid $X$ is an sc-smooth functor $F: X \rightarrow E$ satisfying $P \circ F=\mathrm{id}_{X}$. An sc ${ }^{+}$ section is an sc-smooth section $F: X \rightarrow E$ inducing an sc-smooth functor $X \rightarrow E^{0,1}$, where $E^{0,1}$ has the grading $\left(E^{0,1}\right)_{m}=E_{m, m+1}$ for all $m \geq 0$.

A Fredholm section $F$ of the strong bundle $P: E \rightarrow X$ is an sc-smooth functor which, as a section on the object sets, is an M-polyfold Fredholm section as defined in Definition 3.6 in [22]. 
The functoriality of the section $F: X \rightarrow E$ requires that

$$
\mu(g, F(x))=F(y)
$$

whenever there is a morphism $g: x \rightarrow y$ in $\mathbf{X}$.

The space of sc-smooth sections of the bundle $P: E \rightarrow X$ is denoted by $\Gamma(P)$, the space of $\mathrm{sc}^{+}$-sections is denoted by $\Gamma^{+}(P)$, and the space of Fredholm sections by $\mathcal{F}(P)$. The following observation will be useful later on.

Proposition 2.23 Consider the two strong bundles $P: E \rightarrow X$ and $P^{\prime}: E^{\prime} \rightarrow Y$ over the ep-groupoids $X$ and $Y$ and let $\Phi, \Psi: P^{\prime} \rightarrow P$ be two bundle equivalences which are naturally equivalent. If $F: X \rightarrow E$ is an sc-smooth section of the strong bundle $P$, then the pullback sections $\Phi^{*}(F)$ and $\Psi^{*}(F): Y \rightarrow E^{\prime}$ of the bundle $P^{\prime}$ agree:

$$
\Phi^{*}(F)(y)=\Psi^{*}(F)(y), \quad y \in Y .
$$

Proof Denote by $\varphi, \psi: Y \rightarrow X$ the functors on the base ep-groupoids associated with the bundle equivalences $\Phi, \Psi: P^{\prime} \rightarrow P$, respectively. Then by definition of the pullback of a section we have the equalities

$$
\Phi \circ G(y)=F \circ \varphi(y) \text { and } \Psi \circ H(y)=F \circ \psi(y)
$$

where we have abbreviated $G=\Phi^{*}(F)$ and $H=\Psi^{*}(F)$. If $T: E^{\prime} \rightarrow \mathbf{E}$ is a natural transformation between the bundle maps $\Phi: P^{\prime} \rightarrow P$ and $\Psi: P^{\prime} \rightarrow P$ covering a natural transformation $\tau: Y \rightarrow \mathbf{X}$, then, in view of Definition 2.19,

$$
T(e)=(\tau(P e), \Phi(e)) \text { and } \mu(\tau(P e), \Phi(e))=\Psi(e)
$$

for $e \in E^{\prime}$. Then, since $\tau(y): \varphi(y) \rightarrow \psi(y)$ is a morphism and $F$ a functor, (6) implies that

$$
\begin{aligned}
\Psi(G(y)) & =\mu(\tau(y), \Phi(G(y)) \\
& =\mu(\tau(y), F(\varphi(y))=F(\psi(y)) \\
& =\Psi(H(y))
\end{aligned}
$$

for all $y \in Y$. By assumption, $\Psi$ is a bundle equivalence and $H$ and $G$ are sections. Therefore, $G(y)=H(y)$ for all $y \in Y$ as we wanted to prove.

In order to apply Proposition 2.23, we consider the diagram

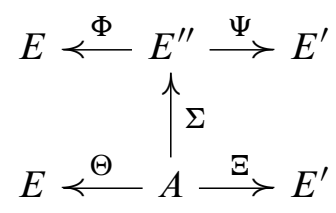


representing a bundle diagram refinement in which all the functors are bundle equivalences. By definition there are the natural equivalences $\Phi \circ \Sigma \simeq \Theta$ and $\Psi \circ \Sigma \simeq \Xi$ and we conclude from Proposition 2.23 for a section $F: X \rightarrow E$ of the strong bundle $P: E \rightarrow X$ over the ep-groupoid $X$ that

$$
(\Phi \circ \Sigma)^{*}(F)=\Theta^{*}(F) .
$$

Similarly, if $G$ is a section of the strong bundle $P^{\prime}: E^{\prime} \rightarrow X^{\prime}$ over the ep-groupoid $X^{\prime}$, then $\Xi^{*}(G)=(\Psi \circ \Sigma)^{*}(G)$ and we can formulate the following definition.

Definition 2.24 Let $P: E \rightarrow X$ and $P^{\prime}: E^{\prime} \rightarrow X^{\prime}$ be two strong bundles over epgroupoids, and let $F, G$ be sections of $P$ and $P^{\prime}$, respectively. The sections $F$ and $G$ are called equivalent, if there exists a diagram

$$
E \stackrel{\Phi}{\leftarrow} E^{\prime \prime} \stackrel{\Psi}{\rightarrow} E^{\prime}
$$

of strong bundle equivalences such that for the pullback sections, we have

$$
\Phi^{*}(F)=\Psi^{*}(G) \text {. }
$$

This is well-defined in the following sense. If we have two equivalent diagrams connecting $E$ with $E^{\prime}$ and the above equality holds for one diagram, then it holds for the other diagram as well. Indeed, by the preceding discussion, it holds for a common refinement and therefore the sections obtained by the pullbacks of the second diagram must be the same on the image of the refinement. But since the latter is a bundle equivalence this implies the assertion.

Also observe that given a bundle equivalence $\Phi: P \rightarrow P^{\prime}$ and a section $F$ of the strong bundle $P: E \rightarrow X$, there is a well-defined push forward section $\Phi_{*}(F)$ of the strong bundle $P^{\prime}: E^{\prime} \rightarrow X^{\prime}$. Consequently, we obtain the following result.

Proposition 2.25 Let $P: E \rightarrow X$ and $P^{\prime}: E^{\prime} \rightarrow X^{\prime}$ be two strong bundles over ep-groupoids. Then an s-invertible generalized bundle map $\mathfrak{A}: E \Rightarrow E^{\prime}$ induces a well-defined pushforward map $\mathfrak{A}_{*}: \Gamma(P) \rightarrow \Gamma\left(P^{\prime}\right)$ between sc-smooth sections which is a bijection. Its inverse is the pullback map $\mathfrak{A}^{*}: \Gamma\left(P^{\prime}\right) \rightarrow \Gamma(P)$. The same holds true for the pushforward maps $\Gamma^{+}(P) \rightarrow \Gamma^{+}\left(P^{\prime}\right)$ for the $\mathrm{sc}^{+}{ }_{-}$sections and $\mathcal{F}(P) \rightarrow \mathcal{F}\left(P^{\prime}\right)$ for the Fredholm sections.

\subsection{Auxiliary norms}

In [22], we introduced the notion of an auxiliary norm for an M-polyfold. In this section we generalize this concept to ep-groupoids by incorporating morphisms. 
We recall from Definition 5.5 in [22] that an auxiliary norm $N$ for the M-polyfold bundle $p: Y \rightarrow X$ consists of a continuous map $N: Y_{0,1} \rightarrow[0, \infty)$ having the following properties:

- For every $x \in X$, the induced map $N \mid\left(Y_{0,1}\right)_{x} \rightarrow[0, \infty)$ on the fiber $\left(Y_{0,1}\right)_{x}$ is a complete norm.

- If $y_{k} \stackrel{m}{\rightarrow} y$, then $N(y) \leq \liminf _{k \rightarrow \infty} N\left(y_{k}\right)$.

- If $N\left(y_{k}\right)$ is a bounded sequence and the underlying sequence $x_{k}=p\left(y_{k}\right)$ converges to $x \in X$, then $y_{k}$ has an $m$-convergent subsequence.

For the so-called $m$-convergence $y_{k} \stackrel{m}{\rightarrow} y$ we refer to Definition 5.4 in [22]. Roughly it means for the sequence $y_{k}=\left(p\left(y_{k}\right), e_{k}\right) \in Y_{0,1}$ that the base points $p\left(y_{k}\right)=x_{k} \in X$ converge in the $\mathrm{M}$-polyfold to $x \in X$ and the fiber components $e_{k} \rightarrow e$ converge in a weak sense to $e \in Y_{1}$ satisfying $p(e)=x$.

Definition 2.26 Let $P: E \rightarrow X$ be a strong bundle over the ep-groupoid $X$. An auxiliary norm for the strong bundle $P$ is a map $N^{*}: E_{0,1} \rightarrow[0, \infty)$ having the following properties:

- As a map on the object set, the map $N^{*}$ is an auxiliary norm as defined above.

- If $\varphi: e \rightarrow e^{\prime}$ is a morphism in $\mathbf{E}$, then $N^{*}(e)=N^{*}\left(e^{\prime}\right)$.

The existence of an auxiliary norm is guaranteed by the following proposition which uses the existence result of Proposition 5.6 in [22]. We assume that the local models for $P$ have reflexive fibers, for example Hilbert spaces.

Proposition 2.27 Every strong bundle $P: E \rightarrow X$ over an ep-groupoid admits an auxiliary norm.

Proof We begin with a strong local bundle $K \rightarrow O$. The existence of an auxiliary norm in this case has been proved in [22, Proposition 5.6]. Next consider a strong bundle $P: E \rightarrow X$ over the ep-groupoid $X$. By the discussion in Section 2.4, we have the strong M-polyfold bundle $P: E \rightarrow X$ over the ep-groupoid $X$ and the strong bundle map $\mu: \mathbf{E} \rightarrow E$ having the property that if $g: x \rightarrow y$ is a morphism in $\mathbf{X}$, then $\mu(g, \cdot): E_{x} \rightarrow E_{y}$ is a linear isomorphism.

Around every point $x \in X$, we choose a strong bundle chart (as in Definition 4.8 in [21])

$$
\Phi: E\left|U_{x} \rightarrow K^{\mathcal{R}}\right| O
$$


covering the sc-diffeomorphism $\varphi: U_{x} \rightarrow O$ between an open neighborhood $U_{x} \subset X$ of the point $x$ and the open subset $O$ of the splicing core $K^{\mathcal{S}}$ associated with the splicing $\mathcal{S}$. The open neighborhood $U_{x}$ can be taken so small that the isotropy group $\mathbf{X}(x)$ acts on it by the natural representation $\varphi_{g}: U_{x} \rightarrow U_{x}$ for $g \in \mathbf{X}(x)$ in view of Theorem 2.3. Since an auxiliary norm exists on $K^{\mathcal{R}} \mid O$, we pull it back to $E \mid U_{x}$ via the chart $\Phi$ to obtain an auxiliary norm $N_{x}^{\prime}$ on $E \mid U_{x}$. We use the following notation. If $y \in X$ and $g: y \rightarrow y^{\prime}$ is a morphism, then we write $y^{\prime}=g \cdot y$. In particular, $y^{\prime}=g \cdot y$ if $y^{\prime}=\varphi_{g}(y)$. Also, we write $e^{\prime}=g \cdot e$ if $e \in E_{x}$ and $e^{\prime}=\mu(g, e) \in E_{y}$. For $\hat{e}=(y, e) \in U_{x} \oplus E_{y}$, we define

$$
\begin{aligned}
\hat{N}_{x}^{\prime}(\widehat{e})=\hat{N}_{x}^{\prime}(y, e) & :=\frac{1}{\sharp \mathbf{X}(x)} \sum_{g \in \mathbf{X}(x)} N_{x}^{\prime}\left(\varphi_{g}(y), \mu(g, e)\right) \\
& =\frac{1}{\sharp \mathbf{X}(x)} \sum_{g \in \mathbf{X}(x)} N_{x}^{\prime}(g \cdot y, g \cdot e) .
\end{aligned}
$$

Then $\hat{N}_{x}^{\prime}$ is an auxiliary norm for $E \mid U_{x}$ having the additional property that if there is a morphism in $\mathbf{E}$ between two objects $\hat{e}$ and $\hat{e}^{\prime}$ belonging to $E \mid U_{x}$, then $\hat{N}_{x}^{\prime}(\widehat{e})=$ $\hat{N}_{x}^{\prime}\left(\widehat{e}^{\prime}\right)$.

Next we extend $\hat{N}_{x}^{\prime}$ to $E \mid \widehat{U}_{x}$ where $\hat{U}_{x}=\pi^{-1}\left(\pi\left(U_{x}\right)\right)$ is the saturation of the set $U_{x}$. Here $\pi: X \rightarrow|X|$ is the quotient map onto the orbits space $|X|$ of the ep-groupoid $X$. If $\hat{e}=(y, e) \in E \mid \widehat{U}_{x}$, then there is a morphism $g$ having the source $y$ and the target in $U_{x}$ such that $\left(y^{\prime}, e^{\prime}\right)=(g \cdot y, g \cdot e) \in U_{x} \oplus E_{y^{\prime}}$ and we define

$$
\widehat{N}_{x}(\widehat{e})=\hat{N}_{x}(y, e):=\hat{N}_{x}^{\prime}(g \cdot y, g \cdot e)=\hat{N}_{x}^{\prime}\left(y^{\prime}, e^{\prime}\right) .
$$

This definition is independent of the choice of a morphism having its source equal to $y$ and its target in $U_{x}$. Indeed, if there is another morphism $g^{\prime}$ satisfying $\left(y^{\prime \prime}, e^{\prime \prime}\right)=$ $\left(g^{\prime} \cdot y, g^{\prime} \cdot e\right) \in U_{x} \oplus E_{y^{\prime}}$, we define the morphism $h=g^{\prime} \circ g^{-1}: y^{\prime} \rightarrow y^{\prime \prime}$. It follows that $\left(y^{\prime \prime}, e^{\prime \prime}\right)=\left(h \cdot y^{\prime}, h \cdot e^{\prime}\right)$, and since $\hat{N}_{x}^{\prime}$ is, by construction, an invariant auxiliary norm for $E \mid \widehat{U}_{x}$, we conclude $\widehat{N}_{x}^{\prime}\left(y^{\prime}, e^{\prime}\right)=\widehat{N}_{x}^{\prime}\left(y^{\prime \prime}, e^{\prime \prime}\right)$ showing that $\hat{N}_{x}$ is well-defined.

To see that the map $\hat{N}_{x}$ is continuous on $E_{0,1} \mid \widehat{U}_{x}$ we take $(x, e) \in E_{0,1} \mid \hat{U}_{x}$. Then there is a morphism $g$ such that $\left(y^{\prime}, e^{\prime}\right)=(g \cdot y, g \cdot e) \in U_{x} \oplus E_{y}$. By definition, $\hat{N}_{x}(x, e)=\hat{N}_{x}^{\prime}\left(y^{\prime}, e^{\prime}\right)$ and $\hat{N}_{x}^{\prime}$ is continuous near $\left(y^{\prime}, e^{\prime}\right)$. The morphism $g$ has an extension to an sc-diffeomorphism of the from $t \circ s^{-1}$ defined near $x$ and the map $\mu$ is also a local sc-diffeomorphism. It follows that the map $\hat{N}_{x}$ near $(y, e)$ is equal to the composition of a continuous map $\hat{N}_{x}^{\prime}$ with the sc-diffeomorphisms $\left(t \circ s^{-1}, \mu \circ\left(t \circ s^{-1}, \mathrm{id}\right)\right)$, and hence is continuous. 
Finally, we glue all the local auxiliary norms $\hat{N}_{x}$ to obtain a globally defined auxiliary norm $N$ for $E$. To do this we consider the open covering $\left(\pi^{-1}\left(\pi\left(U_{x}\right)\right)\right)_{x \in X}$ of $X$. The ep-groupoid $X$ is a paracompact space. In view of Theorem 4.8 in [20], there exists a continuous partition of unity $\left(\beta_{x}\right)_{x \in X}$ subordinated to the open covering $\left(\pi^{-1}\left(\pi\left(U_{x}\right)\right)\right)_{x \in X}$ which is invariant under morphisms of $X$. For simplicity we use here for the partition of unity the same indices as for the open covering, allowing the supports of $\beta_{x}$ to be empty. We would like to point out that Theorem 4.8 in [20] requires the assumption that the sc-structure of $X$ is based on separable sc-Hilbert spaces in order to obtain an sc-smooth partition of unity, however, in our case at hand we don't need this assumption since we are only interested in a continuous partition of unity. Finally we set

$$
N(\widehat{e})=N(y, e)=\sum_{x \in X} \beta_{x}(P(\widehat{e})) \cdot \widehat{N}_{x}(\widehat{e})
$$

for $\hat{e}=(y, e)=(P(\hat{e}), e)$. Then $N$ is the desired auxiliary norm for $E$ which is compatible with the morphisms.

\section{Polyfolds}

Polyfolds and their bundles are the basic spaces on which we will study Fredholm sections. The ep-groupoids can be viewed as the models for this new class of spaces. The strong bundles over ep-groupoids are the models for strong polyfold bundles.

\subsection{Basic definitions and results}

In the following we shall use the category $\mathfrak{E} p$ whose objects are ep-groupoids and whose morphisms are generalized maps.

Definition 3.1 Let $Z$ be a second countable paracompact topological space. A polyfold structure on $Z$ is a pair $(X, \alpha)$ consisting of an ep-polyfold groupoid $X$ and a homeomorphism $\alpha:|X| \rightarrow Z$ between the orbit space and the space $Z$.

Given a polyfold structure $(X, \alpha)$ on $Z$, the $\mathrm{sc}^{0}$-structure on $|X|$ defines an $\mathrm{sc}^{0}-$ structure on $Z$.

To formulate the equivalence relation between two polyfold structures, we recall from the previous section that an s-isomorphism a: $X \Rightarrow Y$ between two ep-groupoids is the equivalence class of a diagram

$$
X \stackrel{F}{\leftarrow} W \stackrel{G}{\rightarrow} Y
$$


connecting ep-groupoids in which both functors $F$ and $G$ are equivalences. Since equivalences induce $\mathrm{sc}^{0}$-homeomorphism between orbit spaces, we obtain the $\mathrm{sc}^{0}-$ homeomorphism $|G| \circ\left|F^{-1}:\right| X|\rightarrow| Y \mid$. This homeomorphism is independent of the choice of a representative in the equivalence class. Indeed, if

$$
X \stackrel{F^{\prime}}{\longleftarrow} W^{\prime} \stackrel{G^{\prime}}{\longrightarrow} Y
$$

is an equivalent diagram, it follows from the definition of the equivalence that

$$
\left|G^{\prime}\right| \circ\left|F^{\prime}\right|^{-1}=|G| \circ|F|^{-1}
$$

and we see that the s-isomorphism $\mathfrak{a}: X \Rightarrow Y$ induces a canonical sc ${ }^{0}$-homeomorphism

$$
|\mathfrak{a}|:|X| \rightarrow|Y|
$$

between the orbit spaces.

Definition 3.2 Let $Z$ be a second countable paracompact topological space. Two polyfold structures $(X, \alpha)$ and $(Y, \beta)$ on $Z$ are called equivalent, $(X, \alpha) \simeq(Y, \beta)$, if there exists an s-isomorphism $\mathfrak{a}: X \Rightarrow Y$ satisfying $\beta \circ|\mathfrak{a}|=\alpha$ :

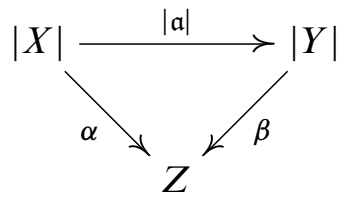

In more detail, two polyfold structures on $Z$ are equivalent if and only if there exists a third ep-groupoid $W$ and two equivalences in the diagram

$$
X \stackrel{F}{\longleftarrow} W \stackrel{G}{\rightarrow} Y
$$

satisfying

$$
\alpha \circ|F|=\beta \circ|G|
$$

Definition 3.3 A polyfold is a second countable paracompact topological space $Z$ equipped with an equivalence class of polyfold structures.

Consider a polyfold $Z$ and a polyfold structure $(X, \alpha)$ on $Z$. The orbit space $|X|$ of the ep-groupoid $X$ is equipped with the filtration $\left|X_{0}\right|=|X| \supset\left|X_{1}\right| \supset \cdots \supset$ $\left|X_{\infty}\right|:=\left|\bigcap_{i \geq 0} X_{i}\right|$ where $\left|X_{\infty}\right|$ is dense in every $\left|X_{i}\right|$. This filtration induces, via the homeomorphism $\alpha:|X| \rightarrow Z$, the filtration

$$
Z_{0}=Z \supset Z_{1} \supset \cdots \supset Z_{\infty}:=\bigcap_{i \geq 0} Z_{i}
$$


on the polyfold $Z$ in which the space $Z_{\infty}$ is dense in every $Z_{i}$. Any other equivalent polyfold structure on $Z$ induces the same filtration. Every space $Z_{i}$ carries a polyfold structure which we denote by $Z^{i}$.

Next we define an sc-smooth map between two polyfolds $Z$ and $Z^{\prime}$. We assume that the polyfold structures of $Z$ and $Z^{\prime}$ are represented by the pairs $(X, \alpha)$ and $\left(X^{\prime}, \alpha^{\prime}\right)$, respectively, and consider a pair $(a, \mathfrak{a})$ consisting of a continuous map $a: Z \rightarrow Z^{\prime}$ and a generalized map a: $X \Longrightarrow X^{\prime}$ satisfying

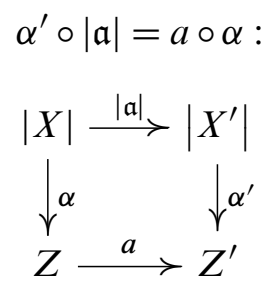

Now we take two other equivalent representatives of the polyfold structures of $Z$ and $Z^{\prime}$, namely $(Y, \beta) \simeq(X, \alpha)$ and $\left(Y^{\prime}, \beta^{\prime}\right) \simeq\left(X^{\prime}, \alpha^{\prime}\right)$. Hence there exist s-isomorphisms

$$
\mathfrak{f}: Y \Longrightarrow X \text { and } \mathfrak{f}^{\prime}: Y^{\prime} \Longrightarrow X^{\prime}
$$

satisfying $\alpha \circ|\mathfrak{f}|=\beta$ and $\alpha^{\prime} \circ\left|\mathfrak{f}^{\prime}\right|=\beta^{\prime}$.

If $(b, \mathfrak{b})$ is another pair consisting of a continuous map $b: Z \rightarrow Z^{\prime}$ and a generalized map $\mathfrak{b}: Y \Longrightarrow Y^{\prime}$ satisfying $\beta^{\prime} \circ|\mathfrak{b}|=b \circ \beta$, we introduce the following definition.

Definition 3.4 The two pairs $(a, \mathfrak{a})$ and $(b, \mathfrak{b})$ are called equivalent provided $a=b$ and $\mathfrak{f}^{\prime} \circ \mathfrak{b}=\mathfrak{a} \circ \mathfrak{f}$.

This defines indeed an equivalence relation. (The composition $\circ$ of two generalized maps is defined in Section 2.3.) The situation is illustrated by the following diagram.

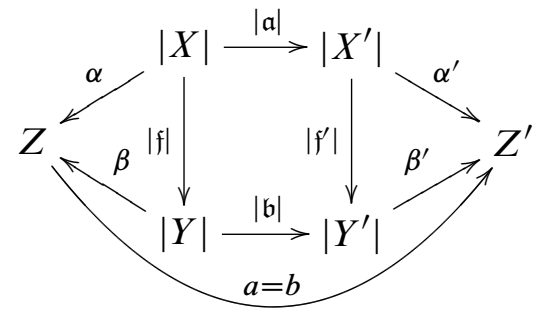

Finally, we can define the notion of an sc-smooth polyfold map.

Definition 3.5 An sc-smooth map $Z \rightarrow Z^{\prime}$ between two polyfolds is an equivalence class $[(a, \mathfrak{a})]$ of pairs consisting of a continuous map $a: Z \rightarrow Z^{\prime}$ between the underlying 
topological spaces and a generalized map

$$
\mathfrak{a}: X \Rightarrow X^{\prime}
$$

where $(X, \alpha)$ and $\left(X^{\prime}, \alpha^{\prime}\right)$ are representatives of the polyfold structures of $Z$ and $Z^{\prime}$, so that

$$
\alpha^{\prime} \circ|\mathfrak{a}|=a \circ \alpha
$$

Sometimes we shall call the map $a: Z \rightarrow Z^{\prime}$ a polyfold map and do not explicitly mention the "overhead" $\mathfrak{a}$ if there is no danger of confusion. So we might say $a: Z \rightarrow$ $Z^{\prime}$ is an sc-smooth map between polyfolds, but the reader must be aware that this is just an abbreviation for a lot of data.

The standard example of a polyfold is the orbit space $|X|$ of an ep-groupoid $X$. The polyfold structure on $|X|$ is given by the pair $\left(X, \operatorname{id}_{|X|}\right)$. Given two such polyfolds $|X|$ and $|Y|$, the space of sc-smooth polyfold maps $|X| \rightarrow|Y|$ is nothing else but all equivalence classes $[(|\mathfrak{a}|, \mathfrak{a})]$ of pairs $(|\mathfrak{a}|, \mathfrak{a})$ in which $\mathfrak{a}$ is a generalized map $X \Rightarrow Y$. A representative (something one can work with if one has to make constructions) for such a generalized map is a diagram

$$
X \stackrel{F}{\leftarrow} A \stackrel{G}{\rightarrow} Y
$$

in which $F$ is an equivalence and $G$ an sc-smooth functor.

In [21] we have introduced the degeneracy index $d: X \rightarrow \mathbb{N}$ on an M-polyfold $X$ as follows. Around a point $x \in X$ we choose an M-polyfold chart $\varphi: U \rightarrow K^{\mathcal{S}}$ where $K^{\mathcal{S}}$ is the splicing core associated with the splicing $\mathcal{S}=(\pi, E, V)$. Here $V$ is an open subset of a partial quadrant $C$ contained in the sc-Banach space $W$. By definition there exists a linear sc-isomorphism from $W$ to $\mathbb{R}^{n} \oplus Q$ mapping $C$ onto $[0, \infty)^{n} \oplus Q$. Identifying the partial quadrant $C$ with $[0, \infty)^{n} \oplus Q$ we shall use the notation $\varphi=\left(\varphi_{1}, \varphi_{2}\right) \in[0, \infty)^{n} \oplus(Q \oplus E)$ according to the splitting of the target space of $\varphi$. We associate with the point $x \in U$ the integer $d(x)$ defined by

$$
d(x)=\sharp\left\{\text { coordinates of } \varphi_{1}(x) \text { which are equal to } 0\right\} .
$$

By Theorem 3.11 in [21], the integer $d$ does not depend on the choice of the M-polyfold chart used. A point $x \in X$ satisfying $d(x)=0$ is called an interior point of $X$. The set $\partial X$ of boundary points of $X$ is defined as

$$
\partial X=\{x \in X \mid d(x)>0\} .
$$

A point $x \in X$ satisfying $d(x)=1$ is called a good boundary point. A point satisfying $d(x) \geq 2$ is called a corner and $d(x)$ is the order of this corner. 
Definition 3.6 The closure of a connected component of the set $X(1)=\{x \in X \mid$ $d(x)=1\}$ is called a face of the $M$-polyfold $X$.

Around every point $x_{0} \in X$ there exists an open neighborhood $U=U\left(x_{0}\right)$ so that every $x \in U$ belongs to precisely $d(x)$ many faces of $U$. This is easily verified. Globally it is always true that $x \in X$ belongs to at most $d(x)$ many faces and the strict inequality is possible.

In order to define the degeneracy index on a polyfold we first look at an ep-polyfold groupoid $X$. Its degeneracy index $d: X \rightarrow \mathbb{N}$ is defined on the M-polyfold $X$ of objects as well as the M-polyfold $\mathbf{X}$ of morphisms. As usual we denote by $s, t: \mathbf{X} \rightarrow X$ the source and target maps of $X$. We have already seen that the existence of a morphism $g: x \rightarrow x^{\prime}$ implies that these three items $x, x^{\prime}$ and $g$ have the same maximal level. The degeneracy map is another integer valued map defined on $X$ having this property.

Lemma 3.7 The following statements hold for ep-polyfold groupoids $X$ and $Y$ :

(i) If $g: x \rightarrow x^{\prime}$ is a morphism, then $d(x)=d\left(x^{\prime}\right)=d(g)$.

(ii) If the functor $F: X \rightarrow Y$ is an equivalence, then $d_{X}(x)=d_{Y}(F(x))$ for all $x \in X$.

Proof Since $s$ and $t$ are local sc-diffeomorphisms, we conclude from the statement about the corner recognition, Proposition 3.13 in [21], that $d(g)=d(s(g))=d(t(g))$. The same proposition implies also the second assertion because the map $F: X \rightarrow Y$ is a local sc-diffeomorphism.

The lemma allows us to define the degeneracy index of the polyfold $Z$. Suppose the pair $(X, \alpha)$ is a polyfold structure on $Z$. If $|x| \in|X|$ and $x, x^{\prime} \in|x|$, then there exists a morphism $g: x \rightarrow x^{\prime}$ and we conclude from Lemma 3.7 that $d(x)=d\left(x^{\prime}\right)$. Hence the map $|d|:|X| \rightarrow \mathbb{N}_{0}$ given by $|d|(|x|)=d(x)$ is well-defined. Using this map, we define the map $d: Z \rightarrow \mathbb{N}_{0}$ on the polyfold by setting

$$
d(z)=|d|\left(\alpha^{-1}(z)\right), \quad z \in Z .
$$

The definition does not depend on the particular choice of the polyfold structure in the equivalence class. To prove this claim we consider two equivalent polyfold structures $(X, \alpha)$ and $(Y, \beta)$ on $Z$. Hence there exists a diagram

$$
X \stackrel{F}{\leftarrow} W \stackrel{G}{\rightarrow} Y
$$

connecting ep-groupoids in which the functors $F$ and $G$ are equivalences satisfying $\alpha \circ|F|=\beta$ 이 $G|:| W \mid \rightarrow Z$. If $\alpha^{-1}(z)=|x|$ and $\beta^{-1}(z)=|y|$, then $|G| \circ|F|^{-1}(|x|)=$ 
$|y|$. Hence we find $w \in W$ such that $|F|(|w|)=|F(w)|=|x|$ and $|G|(|w|)=$ $|G(w)|=|y|$. Therefore, there exists $x^{\prime} \in|x|$ and $y^{\prime} \in|y|$ satisfying $F(w)=x^{\prime}$ and $G(w)=y^{\prime}$ and with Lemma 3.7 we compute,

$$
\begin{aligned}
\left|d_{X}\right|\left(\alpha^{-1}(z)\right) & =\left|d_{X}\right|(|x|)=d_{X}(x) \\
& =d_{X}\left(x^{\prime}\right)=d_{X}(F(w))=d_{W}(w) \\
& =d_{Y}(G(w))=d_{Y}\left(y^{\prime}\right)=d_{Y}(y) \\
& =\left|d_{Y}\right|(|y|)=d_{Y}\left(\beta^{-1}(z)\right)
\end{aligned}
$$

proving the claim.

Definition 3.8 The function $d: Z \rightarrow \mathbb{N}_{0}$ defined by (7) is called the degeneracy index of the polyfold $Z$.

The corner structure of a polyfold will play an important role in the Symplectic Field Theory in [16]. Therefore, we shall look at the faces, introduced in [21], in more detail.

Definition 3.9 A connected component $C$ of an ep-groupoid $X$ is a full subcategory having the following properties:

- If $x \in C$ and if $x^{\prime} \in X$ is in the same component of $X$ as $x$, then $x^{\prime} \in C$.

- If $x \in C$ and $h: x \rightarrow x^{\prime}$ for some $h \in \mathbf{X}$, then $x^{\prime} \in C$.

- The orbit space $|C|$ is connected.

Note that a connected component is an ep-groupoid by definition. We denote by $X(1)$ the set of all good boundary points:

$$
X(1)=\{x \in X \mid d(x)=1\} .
$$

The space $X(1)$ carries in a natural way the structure of an M-polyfold induced by that of $X$. The set of morphisms between points in $X(1)$ is an M-polyfold denoted by $\mathbf{X}(1)$. Together with the structure maps induced from $X$ we obtain the ep-polyfold groupoid $X(1)$. If $C$ is a connected component of this groupoid $X(1)$, then the closure $\bar{C}$ is defined as the full subcategory whose set of objects is the set theoretical closure of the object set $C$. It is called a face of the ep-groupoid $X$. In general, a face need not to have the structure of an ep-groupoid. Given a ep-groupoid we can view a subset $C$ of the object set as a groupoid by taking the full subcategory of $X$ whose objects are the points in $C$. For $C$ as just described we can first take the set theoretic closure $\bar{C}$ and then the associated full subcategory. 
Lemma 3.10 An object $x \in X$ belongs globally to at most $d(x)$-many faces. Locally it belongs to precisely $d(x)$ many faces (this will be made precise during the proof).

Proof Take an object $x \in X$ and let $U(x)$ be an open neighborhood of $x$ which is homeomorphic by means of a chart $(U(x), \varphi,(O, \mathcal{S}))$ to an open set $O$ in a splicing core defined by

$$
\begin{array}{r}
O:=\{((v, f), e) \mid(v, f) \\
\pi_{v}(e)=e, \mid(0, \infty)^{n} \oplus W \text { and } e \in E, \\
=e, \mid<)|<1,| e \mid<1\} .
\end{array}
$$

We may assume that $x$ corresponds to $\left(\left(0, f_{0}\right), e_{0}\right)$ for some $f_{0} \in W$ and some $e_{0} \in E$. Observe that only in the case that $x$ is smooth we may assume that also $f_{0}=0$ and $e_{0}=0$. The set $X(1) \cap U(x)$ corresponds to the points $((v, f), e)$ in $O$ for which $v=\left(v_{1}, \ldots v_{n}\right)$ has precisely one coordinate $v_{j}$ with $1 \leq j \leq n$ vanishing. Define the subset $O_{j} \subset O$ by

$$
O_{j}=\left\{((v, f), e) \in O \mid v_{j}=0 \text { and } v_{i} \neq 0 \text { for } i \in\{1, \ldots, j-1, j+1, \ldots, n\}\right\}
$$

and put $U_{j}=\varphi^{-1}\left(O_{j}\right)$. The closure $O_{j}^{*}$ of $O_{j}$ in $O$ consists of all $((v, f), e) \in O$ having the $j$-coordinate vanishing. It corresponds under $\varphi$ to the closure $U_{j}^{*}$ of $U_{j}$ in $X(1) \cap U(x)$. If a point $((v, f), e) \in O$ satisfies $d((v, f), e)=m \leq n$, then there exist indices $j_{1}, \ldots j_{m} \in\{1, \ldots, n\}$ so that $v_{j_{i}}=0$. Hence the point $((v, f), e)$ belongs to $O_{j_{i}}^{*}$ for all $i=1, \ldots, m$. The converse is also true. Finally we observe that globally, two different (local) closed sets $U_{i}^{*}$ and $U_{j}^{*}$ might belong to the same face. This shows that $x$ belongs to at most $d(x)=m$ faces.

In our applications the so-called face-structured ep-groupoids show up. We introduce this notion in the next definition.

Definition 3.11 An ep-groupoid $X$ is called face-structured if every object $x \in X$ belongs to exactly $d(x)$-many faces.

Lemma 3.12 Let $X$ be a face-structured ep-groupoid and $C$ a connected component of the good boundary points $X(1)$. Then its closure $\bar{C}$ is an ep-groupoid. In other words, the faces are ep-groupoids.

Proof Let $F$ be a face in $X$. Take an object $x_{0} \in F$ and take a chart $(U, \varphi,(O, \mathcal{S}))$ around $x_{0}$. We may assume that

$$
\varphi\left(x_{0}\right)=((0, f), e) \in[0, \infty)^{n} \oplus W \oplus E .
$$

Since $X$ is face-structured $x_{0}$ belongs globally to exactly $d\left(x_{0}\right)$ many faces. This implies that there exists an index $j \in\{1, \ldots, n\}$ so that $F \cap U$ corresponds to all 
points $((v, f), e) \in O$ with $v_{j}=0$. We abbreviate this set by $O_{j}$. We can define in an obvious way a new splicing as follows. We keep $E$, replace $V$ by an open subset $V^{*}$ in $[0, \infty)^{n-1} \oplus W$ and $\pi$ by the family of projections

$$
\rho_{\left(v_{1}, \ldots, v_{j-1}, v_{j+1}, \ldots, v_{n}, f\right)}=\pi_{\left(v_{1}, \ldots, v_{j-1}, 0, v_{j+1}, \ldots, v_{n}, f\right)} .
$$

Now we can use the chart map $\varphi$ to define a chart $\left(U \cap X(1), \varphi,\left(O_{j}, \mathcal{S}^{*}\right)\right)$ with the splicing $\mathcal{S}^{*}=\left(\rho, E, V^{*}\right)$. All charts obtained in this way are sc-smoothly compatible.

Denote by $\mathbf{F}$ the morphism set coming from the full subcategory associated with the face $F$. If a morphism has its source in $F$, then its target is also in $F$ since its degeneracies are the same. Because $s, t: \mathbf{X} \rightarrow X$ are local sc-diffeomorphisms the maps $s, t: \mathbf{F} \rightarrow F$ are local homeomorphisms. The local transition maps $t \circ s^{-1}$ and $s \circ t^{-1}$ are restrictions of sc-smooth maps. Hence we can use them to define a M-polyfold structure on $\mathbf{F}$ for which $s, t: \mathbf{F} \rightarrow F$ are local sc-diffeomorphisms. Finally, the groupoid $F$ is proper. Indeed, if $x$ is an object in $F$ we take an open neighborhood $U \subset X$ of $x$ so that

$$
t: s^{-1}(\bar{U}) \rightarrow X
$$

is proper. Taking $U^{\prime}:=U \cap F$ the result follows because the object set $F$ is closed in $X$.

Next we study the geometry of faces in more detail.

Lemma 3.13 Let $F: X \rightarrow Y$ be an equivalence between two ep-groupoids. Assume that $D$ is a connected component of $Y(1)$. Then there exists a unique connected component $C$ of $X(1)$ so that for every $y \in D$ there is a morphism $\varphi: F(x) \rightarrow y$ for some $x \in C$. Conversely, if $C$ is a connected component of $X(1)$, then there exists a unique connected component $D$ of $Y(1)$ satisfying $F(C) \subset D$.

Proof Let $D$ be a connected component of $Y(1)$ and $y_{0} \in D$. Since $F: X \rightarrow Y$ is an equivalence, the map $|F|:|X| \rightarrow|Y|$ is an homeomorphism. Hence there exists a morphism $\varphi_{0}: F\left(x_{0}\right) \rightarrow y_{0}$ for some $x_{0} \in X$. From Lemma 3.7 we deduce $d\left(x_{0}\right)=d\left(y_{0}\right)=1$ implying that $x_{0} \in X(1)$. Define the subset $C$ of the object set by

$$
C=\{x \in X(1) \mid \text { there are an } y \in D \text { and a morphism } \varphi: F(x) \rightarrow y\} .
$$

We claim that $C$ is a connected component of $X(1)$. Take $x \in C$ and assume that $x^{\prime} \in X(1)$ belongs to a path component of $X(1)$. Then there is a continuous path $\alpha:[0,1] \rightarrow X(1)$ such that $\alpha(0)=x$ and $\alpha(1)=x^{\prime}$. Consider the continuous path $\beta=F \circ \alpha$. Then $\beta(0)=F(x)$ and $\beta(1)=F\left(x^{\prime}\right)$. Since $x \in C$, there exists $y \in D$ and a morphism $\varphi_{0}: F(x) \rightarrow y$. By the definition of the connected component $D$ 
we have $F(x) \in D$ and hence $F\left(x^{\prime}\right) \in D$. This implies that $x^{\prime} \in C$. If $x \in C$ and $h: x \rightarrow x^{\prime}$ is a morphism, then $F(h): F(x) \rightarrow F\left(x^{\prime}\right)$. From $F(x) \in D$ we conclude $F\left(x^{\prime}\right) \in D$, and consequently $x^{\prime} \in C$. Finally, $|C|:=|F|^{-1}(|D|)$ is connected since $|F|:|X| \rightarrow|Y|$ is a homeomorphism and $|D|$ is connected.

Conversely, assume that $C$ is a connected component of $X(1)$. Define the subset

$$
D=\{y \in Y(1) \mid \text { there are an } x \in C \text { and a morphism } \varphi: F(x) \rightarrow y\} .
$$

Then $F(C) \subset D$ and we claim that $D$ is a connected component of $Y(1)$. If $y \in D$ and $y^{\prime}$ belongs to the path component of $Y(1)$, then there is a continuous path $\beta:[0,1] \rightarrow$ $Y(1)$ such that $\beta(0)=y$ and $\beta(1)=y^{\prime}$. By the definition of $D$, there is $x \in C$ and a morphism $\varphi: F(x) \rightarrow y$. Since $F$ is an equivalence, we find a continuous path $\alpha:[0,1] \rightarrow X$ such that $|F| \circ \pi_{X} \circ \alpha=\pi_{Y} \circ \beta$. Since $C$ is the connected component, the point $x^{\prime}=\alpha(1)$ belongs to $C$ and since there is a morphism $\varphi: F\left(x^{\prime}\right) \rightarrow y^{\prime}$, the point $y^{\prime}$ belongs to $D$. Next assume $y \in D$ and there is a morphism $h: y \rightarrow y^{\prime}$. Then there are $x \in C$ and a morphism $\varphi: F(x) \rightarrow y$. Hence $h \circ \varphi: F(x) \rightarrow y^{\prime}$ is a morphism showing that $y^{\prime} \in D$. Also, $|D|=|F|(|C|)$ showing that $|D|$ is connected. We have proved that the equivalence $F: X \rightarrow Y$ induces a bijective map between connected components of $X(1)$ and connected components of $Y(1)$ by

$$
C \leftrightarrow m,
$$

where we associate with a connected component $C$ the connected component $D$ of $Y(1)$ satisfying $F(C) \subset D$ and with a connected component $D$ the connected component $C$ consisting of those $x \in X(1)$ for which there is a morphism between $F(x)$ and a point in $D$.

If $F: X \rightarrow Y$ is an equivalence and $C$ a connected component of $X(1)$, we denote by $F_{*}(C)$ the component of $Y(1)$ containing $F(C)$. If $D$ is a connected component of $Y(1)$, we denote by $F^{*}(D)$ the component of $X(1)$ mapped into $D$. We denote by $\bar{C}$ the closure of the connected component $C$ of $X(1)$ and similarly by $\bar{D}$ the closure of the connected component $D$ in $Y(1)$.

Lemma 3.14 If $C$ is a connected component of $X(1)$, then $F(\bar{C}) \subset \overline{F_{*}(C)}$. If $D$ is a connected component of $Y(1)$, then the set

$$
W=\{x \in X(1) \mid \text { there are an } y \in \bar{D} \text { and a morphism } \varphi: F(x) \rightarrow y\}
$$

is contained in $\overline{F^{*}(D)}$. In other words, $F_{*}$ and $F^{*}$ map faces to faces.

Proof Take $x \in \bar{C}$ and a sequence $\left(x_{n}\right) \subset C$ converging to $x$. Then $F\left(x_{k}\right) \in F_{*}(C)$ and $F\left(x_{k}\right) \rightarrow F(x)$ implying that $F(x) \in \overline{F_{*}(C)}$. Next take $x \in W$. Then there is 
$y \in \bar{D}$ and a morphism $\varphi: F(x) \rightarrow y$. Since $F$ is a local sc-diffeomorphism, we find open neighborhoods $U(x) \subset X$ and $U(F(x)) \subset Y$ of the points $x$ and $F(x)$ such that $F: U(x) \rightarrow U(F(x))$ is an sc-diffeomorphism. The morphism $\varphi: F(x) \rightarrow y$ extends to a local sc-diffeomorphism of the form $\hat{\varphi}=t \circ s^{-1}$. Shrinking the set $U(x)$ if necessary, we may assume that $\hat{\varphi}: U(F(x)) \rightarrow U(y)$ is an sc-diffeomorphism. Take any sequence $\left(y_{n}\right) \subset D$ such that $y_{n} \rightarrow y$. Then $\left(y_{n}\right) \subset U(y)$ and we find a sequence $\left(x_{n}\right) \in U(x)$ such that $x_{n} \rightarrow x$ and $\hat{\varphi}\left(F\left(x_{n}\right)\right)=y_{n}$. By Lemma 3.7, $d\left(x_{n}\right)=d\left(y_{n}\right)=1$ and since $\hat{\varphi}$ defines a morphism between $F\left(x_{n}\right)$ and $y_{n}$, we conclude that $x_{n} \in F^{*}(D)$. This implies $x \in \overline{F^{*}(D)}$ as claimed.

We summarize the previous discussion in the following proposition.

Proposition 3.15 Assume that $F: X \rightarrow Y$ is an equivalence between ep-groupoids. Then the maps $F_{*}$ and $F^{*}$ map faces to faces and are mutual inverses. In particular, if one of the ep-groupoids is face-structured so is the other.

Proof It only remains to prove the last statement. We assume that $X$ is face-structured and prove that $Y$ is also face-structured. Take a point $y \in Y$. Then there is $x \in X$ and a morphism $\varphi: F(x) \rightarrow y$. By Lemma 3.7, $d:=d(x)=d(y)$. Since $X$ is face-structured, the point $x$ belongs to $d$-many faces. By Lemma 3.14, the map $F_{*}$ takes these faces onto exactly $d$-many faces of $Y$ containing the point $y$. Conversely, assume that $Y$ is face-structured. Take a point $x \in X$ and set $y=F(x)$. Then $d:=d(x)=d(y)$ and since $Y$ is face-structured, the point $y$ belongs to exactly $d$ faces. Then the map $F^{*}$ takes these faces to $d$-many faces of $X$ containing the point $x$. Hence $X$ is face-structured and the proof of the proposition is complete.

Proposition 3.16 Let $X$ be a face-structured ep-groupoid. Then the intersection of an arbitrary number of faces carries in a natural way the structure of an ep-groupoid.

Proof The ep-structure on a finite intersection of faces can be defined as we did for a face in Lemma 3.12.

We next consider two pairs $(X, \alpha)$ and $\left(X^{\prime}, \alpha^{\prime}\right)$ of equivalent polyfold structures on polyfold $Z$. This means that there exist a third ep-groupoid $X^{\prime \prime}$ and two equivalences $F: X^{\prime \prime} \rightarrow X$ and $F^{\prime}: X^{\prime \prime} \rightarrow X^{\prime}$ satisfying $\alpha \circ|F|=\alpha^{\prime} \circ\left|F^{\prime}\right|$. If $D$ is a connected component of $X(1)$, then by Lemma $3.14, F^{*}(D)$ is the associated connected component of $X^{\prime \prime}(1)$ and $D^{\prime}=F_{*}^{\prime} \circ F^{*}(D)$ the connected component of $X^{\prime}(1)$. Hence there is a one-one correspondence between the faces of $X(1)$ and $X^{\prime}(1)$. In particular, if the ep-groupoid $X$ is face-structured, then the same is true for the ep-groupoid $X^{\prime}$. 
If $C$ is a face of $X(1)$ and $C^{\prime}=F_{*}^{\prime} \circ F^{*}(C)$ is the corresponding face of $X^{\prime}(1)$, then $|F|^{-1}(|C|)=\left|F^{\prime}\right|^{-1}\left(\left|C^{\prime}\right|\right)$ and

$$
\alpha^{\prime}\left(\left|C^{\prime}\right|\right)=\alpha^{\prime} \circ\left|F^{\prime}\right| \circ|F|^{-1}(|C|)=\alpha \circ|F| \circ|F|^{-1}(|C|)=\alpha(|C|) .
$$

This allows to introduce the concepts of a face and of face-structured polyfolds.

Definition 3.17 Let $Z$ be a polyfold and let $(X, \alpha)$ be a polyfold structure on $Z$. The polyfold $Z$ is said to be face-structured if the ep-groupoid $X$ is face-structured. A face $D$ of the polyfold $Z$ is the image $D=\alpha(|C|)$ of the orbit space of a face $C$ in $X$.

\subsection{Branched suborbifolds}

In this section we shall introduce the notion of a branched suborbifold of a polyfold $Z$ and start with the definition of a branched ep-subgroupoid of an ep-groupoid $X$. It generalizes ideas from [2] where quotients of manifolds by global group-actions are studied.

We shall view the nonnegative rational numbers, denoted by $\mathbb{Q}^{+}=\mathbb{Q} \cap[0, \infty)$, as the objects in a category having only the identities as morphisms. We would like to mention that branched ep-subgroupoids will show up as solution sets of polyfold Fredholm sections.

Definition 3.18 A branched ep-subgroupoid of the ep-groupoid $X$ is a functor

$$
\Theta: X \rightarrow \mathbb{Q}^{+}
$$

having the following properties:

(1) The support of $\Theta$, defined by supp $\Theta=\{x \in X \mid \Theta(x)>0\}$, is contained in $X_{\infty}$

Every point $x \in \operatorname{supp} \Theta$ is contained in an open neighborhood $U(x)=U \subset X$ such that:

$$
\operatorname{supp} \Theta \cap U=\bigcup_{i \in I} M_{i}
$$

where $I$ is a finite index set and where the sets $M_{i}$ are finite dimensional submanifolds of $X$ (in the sense of Definition 4.19 in [22], recalled in the Appendix, Section 5.3) all having the same dimension, and all in good position to the boundary $\partial X$ in the sense of Definition 4.14 in [22]. The submanifolds $M_{i}$ are called local branches in $U$. 
(3) There exist positive rational numbers $\sigma_{i}, i \in I$, (called weights) such that if $y \in \operatorname{supp} \Theta \cap U$, then

$$
\Theta(y)=\sum_{\left\{i \in I \mid y \in M_{i}\right\}} \sigma_{i} .
$$

(4) The inclusion maps $M_{i} \rightarrow U$ are proper.

(5) There is a natural representation of the isotropy group $\mathbf{X}(x)$ acting by scdiffeomorphisms on $U$.

The branches $\left(M_{i}\right)_{i \in I}$ together with the weights $\left(\sigma_{i}\right)_{i \in I}$ constitute a local branching structure of $X$ in $U$. The role of local branchings structures and their properties have been studied in detail in [20] where also the notion of an orientation for a branched ep-subgroupoid as well as for a branched suborbifold is introduced.

Remark Given a branched ep-subgroupoid $\Theta$ of the ep-groupoid $X$ we obtain the induced ep-subgroupoids $\Theta^{i}: X^{i} \rightarrow \mathbb{Q}^{+}$for $i \geq 1$. Observe that the supports of $\Theta=\Theta^{0}$ and $\Theta^{i}$ coincide. The reader should notice that all upcoming constructions carried out for $\Theta$ will lead to the same result if carried out for $\Theta^{i}$.

Definition 3.19 A branched ep-subgroupoid is called $n$-dimensional if all its local branches are $\mathrm{n}$-dimensional submanifolds of $M$. It is called compact if $|\operatorname{supp} \Theta|$ is compact.

From the assumption that $\Theta: X \rightarrow \mathbb{Q}^{+}$is a functor it follows that

$$
\Theta(x)=\Theta(y)
$$

if there is a morphism $\varphi: x \rightarrow y$. Therefore, a branched ep-subgroupoid $\Theta$ induces a canonical map $|\Theta|:|X| \rightarrow \mathbb{Q}^{+}$defined as

$$
|\Theta|(|x|)=\Theta(x) .
$$

Next we recall the definition of an orientation from [20].

Definition 3.20 Let $\Theta: X \rightarrow \mathbb{Q}^{+}$be a branched ep-subgroupoid on the ep-groupoid $X$ and $S$ its support. An orientation for $\Theta$, denoted by $\mathfrak{o}$, consists of an orientation for every local branch of the tangent set $T_{x} S$ at every point $x \in S$ so that the following compatibility conditions are satisfied:

- At every point $x$, there exists a local branching structure $\left(M_{i}\right)_{i \in I}$ where the finite dimensional submanifolds $M_{i}$ of $X$ can be oriented in such a way that the orientations of $T_{x} M_{i}$ induced from $M_{i}$ agree with the given ones for the local branches of the tangent set $T_{x} S$. 
- If $\varphi: x \rightarrow y$ is a morphism between two points in $S_{\Theta}$, then the tangent map $T \varphi: T_{x} S \rightarrow T_{y} S$ maps every oriented branch to an oriented branch preserving the orientation.

Next we consider triples $(X, \alpha, \Theta)$ in which the pair $(X, \alpha)$ is a polyfold structure on $Z$ and $\Theta: X \rightarrow \mathbb{Q}^{+}$is a branched ep-subgroupoid of the ep-groupoid $X$. Two such triples $(X, \alpha, \Theta)$ and $\left(X^{\prime}, \alpha^{\prime}, \Theta^{\prime}\right)$ are called equivalent if there exists a third triple $\left(X^{\prime \prime}, \alpha^{\prime \prime}, \Theta^{\prime \prime}\right)$ and two equivalences

$$
X \stackrel{F}{\leftarrow} X^{\prime \prime} \stackrel{F^{\prime}}{\longrightarrow} X^{\prime}
$$

satisfying $\alpha^{\prime \prime}=\alpha \circ|F|=\alpha^{\prime} \circ\left|F^{\prime}\right|$ for the induced maps $|F|$ and $\left|F^{\prime}\right|$ on the orbit spaces, and in addition,

$$
\Theta^{\prime \prime}=\Theta^{\prime} \circ F^{\prime}=\Theta \circ F .
$$

We denote by $[(X, \alpha, \Theta)]$ the equivalence class of the triple $(X, \alpha, \Theta)$. Since $F, F^{\prime}$ and $\Theta, \Theta^{\prime}$ are functors, we conclude that

$$
\left|\Theta^{\prime \prime}\right|=|\Theta| \circ|F|=|\Theta| \circ|F| \text {. }
$$

This implies that $|F|(|\operatorname{supp} \Theta|)=\left|F^{\prime}\right|\left(\left|\operatorname{supp} \Theta^{\prime}\right|\right)$ and since $\alpha^{\prime \prime}=\alpha \circ|F|=\alpha^{\prime} \circ\left|F^{\prime}\right|$, we conclude that

$$
\alpha(|\operatorname{supp} \Theta|)=\alpha^{\prime}\left(\left|\operatorname{supp} \Theta^{\prime}\right|\right) \text {. }
$$

In addition, since $|\Theta| \circ \alpha^{-1}=\left|\Theta^{\prime}\right| \circ\left(\alpha^{\prime}\right)^{-1}$, the function $\vartheta: Z \rightarrow \mathbb{Q}^{+}$defined by $\vartheta(z)=|\Theta|\left(\alpha^{-1}(z)\right)$ does not depend on the choice of the triple $(X, \alpha, \Theta)$ in its equivalence class and satisfies

$$
\vartheta(\alpha(|x|)=\Theta(x) \text { for all } x \in X .
$$

This prompts the following definition.

Definition 3.21 A branched suborbifold of the polyfold $Z$ is an equivalence class $[(X, \alpha, \Theta)]$.

Associated with $[(X, \alpha, \Theta)]$ we have the subset $S$ contained in $Z_{\infty}$ defined by

$$
S=\alpha(|\operatorname{supp} \Theta|)
$$

and the function $\vartheta: Z \rightarrow \mathbb{Q}^{+}$, called weight function, defined by

$$
\vartheta(\alpha(|x|))=\Theta(x) \text {. }
$$

Observe that $S$ consists of all points $z \in Z$ such that $\vartheta(z)>0$. On should also view $(S, \vartheta \mid S)$ as a weighted space. Quite often we shall identify $\vartheta$ with the equivalence class $\vartheta=[(X, \alpha, \Theta)]$. 
The diagram

$$
X \stackrel{F}{\longleftarrow} X^{\prime \prime} \stackrel{F^{\prime}}{\longrightarrow} X^{\prime}
$$

above defines the s-isomorphism

$$
\mathfrak{a}=\left[X \stackrel{F}{\leftarrow} X^{\prime \prime} \stackrel{F^{\prime}}{\longrightarrow} X^{\prime}\right]: X \Rightarrow X^{\prime} .
$$

Then $\alpha^{\prime} \circ|\mathfrak{a}|=\alpha$ for the induced map $|\mathfrak{a}|=\left|F^{\prime}\right| \circ|F|^{-1}:|X| \rightarrow\left|X^{\prime}\right|$. Consequently, the identity $\left|\Theta^{\prime \prime}\right|=|\Theta| \circ|F|=|\Theta| \circ|F|$, gives following relation between branched ep-subgroupoid $\Theta$ and $\Theta^{\prime}$ in equivalent triples $(X, \alpha, \Theta)$ and $\left(X^{\prime}, \alpha^{\prime}, \Theta^{\prime}\right)$.

$$
\left|\Theta^{\prime}\right| \circ|\mathfrak{a}|=|\Theta| \text {. }
$$

The branched suborbifold $S$ of the polyfold $Z$ is called $n$-dimensional, resp. compact, resp. oriented, if the representative $(X, \alpha, \Theta)$ of the ep-groupoid is $n$-dimensional, resp. compact, resp. oriented. In the latter case the equivalences $F$ and $F^{\prime}$ involved are required to preserve the orientations.

\subsection{Branched integration}

There is a canonical measure on an oriented branched suborbifold of a polyfold and an associated integration theory which we would like to describe briefly in this section referring to [20] for details and proofs.

The measure will be induced from the overhead $(X, \alpha, \Theta)$ where $\Theta: X \rightarrow \mathbb{Q}^{+}$is a branched ep-subgroupoid of the ep-groupoid $X$ of dimension $n$. We assume that the orbit space of $\Theta$, namely $S=|\operatorname{supp} \Theta|$, is compact. We abbreviate the orbit space of the boundary by

$$
\partial S=|\operatorname{supp} \Theta \cap \partial X|=\{|x| \in S \mid x \in \operatorname{supp} \Theta \cap \partial X\} .
$$

The orbit space $\partial S$, in general, is not necessarily the boundary of $S$ in the sense of topology. Locally the sets $S$ and $\partial S$ can be represented as quotients of finite unions of smooth submanifolds by a sc-smooth group action on the ambient space. This structure suffices to generalize the familiar notion of the Lebesgue $\sigma$-algebra of subsets of smooth manifolds as follows.

Theorem 3.22 (Canonical $\sigma$-algebra) The compact topological spaces $S$ and $\partial S$ possess canonical $\sigma$-algebras $\mathcal{L}(S)$ and $\mathcal{L}(\partial S)$ of subsets containing the Borel $\sigma-$ algebras of $S$ and $\partial S$, respectively. 
The sets belonging to $\mathcal{L}(S)$ and $\mathcal{L}(\partial S)$ are called measurable. The precise definition of the $\sigma$-algebras is given in [20]. The canonical $\sigma$-algebras for the branched epsubgroupoid $\Theta$ and the induced ep-subgroupoids $\Theta^{i}$ are identical.

If $X$ is an ep-groupoid, then the tangent space $T X$ is again an ep-groupoid. Recall that $T X$ is defined by equivalence classes $[U, \varphi,(O, \mathcal{S}), x, h]$, where $x \in X^{1}, \varphi: U \rightarrow O$ is a chart where $O$ is open in the splicing core $K^{\mathcal{S}}$ and $h \in T_{X} O$. The map $T X \rightarrow X^{1}$ is given by $[U, \varphi,(O, \mathcal{S}), x, h] \mapsto x$. If $\psi: x \rightarrow y$ is a morphism between points $x, y \in X_{1}$, then $\psi$ belongs to $\mathbf{X}_{1}$ and extends to an sc-diffeomorphism $t \circ s^{-1}: O(x) \rightarrow O(y)$. We define $T \psi: T_{x} X \rightarrow T_{y} X$ to be the linearization of $t \circ s^{-1}$ at $x$. Hence $T X$ is an ep-groupoid whose structure maps are the tangents of the structure maps of $X$. If $F:(X, \alpha) \rightarrow\left(X^{\prime}, \alpha^{\prime}\right)$ is an equivalence of polyfold structures, then $T F: T X \rightarrow$ $T X^{\prime}$ is an equivalence. It also defines an equivalence between the Whitney sums $\bigoplus_{k} T X \rightarrow \bigoplus_{k} T X^{\prime}$ covering the equivalence $F: X \rightarrow X^{\prime}$. In the following we denote by $\bigoplus_{k} T X \rightarrow X^{1}$ the Whitney sum of $k$-many copies of $T X$.

At this point we would like to make some comments about our notation. For us the tangent bundle of $X$ is $T X \rightarrow X^{1}$, that is, it is only defined for the base points in $X^{1}$. An sc-vector field on $X$ is an sc-smooth section of the tangent bundle $T X \rightarrow X^{1}$ and hence it is defined on $X^{1}$. Similarly, an sc-differential form on $X$ which we will define next, is only defined over the base points in $X^{1}$. The definition of a vector field and the following definition of an sc-differential form are justified since the construction of $T X$, though only defined over $X^{1}$, requires the knowledge of $X$.

Definition 3.23 An sc-differential $k$-form on the ep-groupoid $X$ is an sc-smooth map $\omega: \bigoplus_{k} T X \rightarrow \mathbb{R}$ which is linear in each argument separately and skew symmetric. In addition, we require that

$$
(T \varphi)^{*} \omega_{y}=\omega_{x}
$$

for all morphisms $\varphi: x \rightarrow y$ in $\mathbf{X}_{1}$.

If $\omega$ is an sc-differential form on $X$, we may also view it as an sc-differential form on $X^{i}$. We denote by $\Omega^{*}\left(X^{i}\right)$ the graded commutative algebra of sc-differential forms on $X^{i}$. Then we have the inclusion map

$$
\Omega^{*}\left(X^{i}\right) \rightarrow \Omega^{*}\left(X^{i+1}\right) .
$$

which is injective since $X_{i+1}$ is dense in $X_{i}$ and the forms are sc-smooth. Hence we have a directed system whose direct limit is denoted by $\Omega_{\infty}^{*}(X)$. An element $\omega$ of degree $k$ in $\Omega_{\infty}^{*}(X)$ is a skew-symmetric map $\bigoplus_{k}(T X)_{\infty} \rightarrow \mathbb{R}$ such that it has an sc-smooth extension to an sc-smooth k-form $\bigoplus_{k} T X^{i} \rightarrow \mathbb{R}$ for some $i$. We shall refer to an element of $\Omega_{\infty}^{k}(X)$ as an sc-smooth differential form on $X_{\infty}$. We note, 
however, that it is part of the structure that the $k$-form is defined and sc-smooth on some $X^{i}$.

Next we associate with an sc-differential $k$-form $\omega$ its exterior differential $d \omega$ which is a $(k+1)$-form on the ep-groupoid $X^{1}$. The compatibility with morphisms will be essentially an automatic consequence of the definition. Hence it suffices for the moment to consider the case that $X$ is an M-polyfold. Let $A_{0}, \ldots, A_{k}$ be $k+1$ many sc-smooth vector fields on $X$. We define $d \omega$ on $X^{1}$, using the familiar formula, by

$$
\begin{aligned}
d \omega\left(A_{0}, \ldots, A_{k}\right)= & \sum_{i=0}^{k}(-1)^{i} D\left(\omega\left(A_{0}, \ldots, \widehat{A}_{i}, \ldots, A_{k}\right)\right) \cdot A_{i} \\
& +\sum_{i<j}(-1)^{(i+j)} \omega\left(\left[A_{i}, A_{j}\right], A_{0}, \ldots, \widehat{A}_{i}, \ldots, \widehat{A}_{j}, \ldots, A_{k}\right) .
\end{aligned}
$$

The right-hand side of the formula above only makes sense at the base points $x \in X_{2}$. This explains why $d \omega$ is a $(k+1)$-form on $X^{1}$. By the previous discussion the differential $d$ defines a map

$$
d: \Omega^{k}\left(X^{i}\right) \rightarrow \Omega^{k+1}\left(X^{i+1}\right)
$$

and consequently induces a map

$$
d: \Omega_{\infty}^{*}(X) \rightarrow \Omega_{\infty}^{*+1}(X)
$$

having the usual property $d^{2}=0$. Then $\left(\Omega_{\infty}^{*}(X), d\right)$ is a graded differential algebra which we shall call the de Rham complex.

If $\varphi: M \rightarrow X$ is an sc-smooth map from a finite-dimensional manifold $M$ into an $\mathrm{M}$-polyfold $X$, then it induces an algebra homomorphism

satisfying

$$
\begin{gathered}
\varphi^{*}: \Omega_{\infty}^{*}(X) \rightarrow \Omega_{\infty}^{*}(M) \\
d\left(\varphi^{*} \omega\right)=\varphi^{*} d \omega .
\end{gathered}
$$

To formulate the next theorem we recall the natural representation $\varphi: \mathbf{G}(x) \rightarrow \operatorname{Diff}_{\mathrm{sc}}(U)$ of the isotropy group $\mathbf{G}(x)=G$. This group can contain a subgroup acting trivially on $U$. Such a subgroup is a normal subgroup of $G$ called the ineffective part of $G$ and denoted by $G_{0}$. The effective part of $G$ is the quotient group

$$
G_{\mathrm{e}}:=G / G_{0} .
$$

We denote the order of $G_{\mathrm{e}}$ by $\sharp G_{\mathrm{e}}$. 
Theorem 3.24 (Canonical measures) Let $X$ be an ep-groupoid and assume that $\Theta: X \rightarrow \mathbb{Q}^{+}$is an oriented branched ep-subgroupoid of dimension $n$ whose orbit space $S=|\operatorname{supp} \Theta|$ is compact and equipped with the weight function $\vartheta: S \rightarrow \mathbb{Q}^{+}$ defined by

$$
\vartheta(|x|):=\Theta(x), \quad|x| \in S .
$$

Then there exists a map

$$
\Phi_{(S, \vartheta)}: \Omega_{\infty}^{n}(X) \rightarrow \mathcal{M}(S, \mathcal{L}(S)), \quad \omega \mapsto \mu_{\omega}^{(S, \theta)}
$$

which associates to every sc-differential $n$-form $\omega$ on $X_{\infty}$ a signed finite measure

$$
\mu_{\omega}^{(S, \vartheta)} \equiv \mu_{\omega}
$$

on the canonical measure space $(S, \mathcal{L}(S))$. This map is uniquely characterized by the following properties:

(1) The map $\Phi_{(S, \theta)}$ is linear.

(2) If $\alpha=f \tau$ where $f \in \Omega_{\infty}^{0}(X)$ and $\tau \in \Omega_{\infty}^{n}(X)$, then

$$
\mu_{\alpha}(K)=\int_{K} f d \mu_{\tau}
$$

for every set $K \subset S$ in the $\sigma$-algebra $\mathcal{L}(S)$.

(3) Given a point $x \in \operatorname{supp} \Theta$ and an oriented branching structure $\left(M_{i}\right)_{i \in I}$ with the associated weights $\left(\sigma_{i}\right)_{i \in I}$ on the open neighborhood $U$ of $x$ according to Definition 3.18, then for every set $K \in \mathcal{L}(S)$ contained in a compact subset of |supp $\Theta \cap U \mid$, the $\mu_{\omega}$-measure of $K$ is given by the formula

$$
\mu_{\omega}(K)=\frac{1}{\sharp G_{e}} \sum_{i \in I} \sigma_{i} \int_{K_{i}} \omega \mid M_{i}
$$

where $K_{i}$ is the preimage of $K$ under the projection map $M_{i} \rightarrow|\operatorname{supp} \Theta \cap U|$ defined by $x \rightarrow|x|$.

In the theorem above we denoted by $\int_{K_{i}} \omega \mid M_{i}$ the signed measure of the set $K_{i}$ with respect to the Lebesgue signed measure associated with the smooth $n$-form $\omega \mid M_{i}$ on the finite dimensional manifold $M_{i}$. It is given by

$$
\int_{K_{i}} \omega \mid M_{i}=\lim _{k} \int_{U_{k}} j^{*} \omega
$$

where $j: M_{i} \rightarrow X$ is the inclusion map and $\left(U_{k}\right)$ is a decreasing sequence of open neighborhoods of $K_{i}$ in $M_{i}$ satisfying $\bigcap_{k} U_{k}=K_{i}$. 
The analogous result holds true for the orbit space $\partial S=\{|x| \in S \mid x \in \operatorname{supp} \Theta \cap \partial X\}$ of the boundary.

Theorem 3.25 (Canonical boundary measures) Under the same assumptions as in Theorem 3.24 there exists a map

$$
\Phi_{(\partial S, \vartheta)}: \Omega_{\infty}^{n-1}(X) \rightarrow \mathcal{M}(\partial S, \mathcal{L}(\partial S)), \quad \tau \mapsto \mu_{\tau}^{(\partial S, \vartheta)}
$$

which assigns to every sc-differential $(n-1)$-form $\tau$ on $X_{\infty}$ a signed finite measure

$$
\mu_{\tau}^{(\partial S, \vartheta)} \equiv \mu_{\tau}
$$

on the canonical measure space $(\partial S, \mathcal{L}(\partial S))$. This map is uniquely characterized by the following properties:

(1) The map $\Phi_{(\partial S, \vartheta)}$ is linear.

(2) If $\alpha=f \tau$ where $f \in \Omega_{\infty}^{0}(X)$ and $\tau \in \Omega_{\infty}^{n-1}(X)$, then every $K \in \mathcal{L}(\partial S)$ has the $\mu_{\alpha}$-measure

$$
\mu_{\alpha}(K)=\int_{K} f d \mu_{\tau}
$$

(3) Given a point $x \in \operatorname{supp} \Theta \cap \partial X$ and an oriented branching structure $\left(M_{i}\right)_{i \in I}$ with weights $\left(\sigma_{i}\right)_{i \in I}$ on the open neighborhood $U \subset X$ of $x$, then the measure of $K \in \mathcal{L}(\partial S)$ contained in a compact subset of $|\operatorname{supp} \Theta \cap U \cap \partial X|$ is given by the formula

$$
\mu_{\tau}(K)=\frac{1}{\sharp G_{e}} \sum_{i \in I} \sigma_{i} \int_{K_{i}} \tau \mid \partial M_{i}
$$

where $K_{i} \subset \partial M_{i}$ is the preimage of $K$ under the projection map $\partial M_{i} \rightarrow$ $|\operatorname{supp} \Theta \cap U \cap \partial X|$ defined by $x \mapsto|x|$.

Finally, the following version of Stokes' theorem holds.

Theorem 3.26 (Stokes' theorem) Let $X$ be an ep-groupoid and let $\Theta: X \rightarrow \mathbb{Q}^{+}$ be an oriented $n$-dimensional branched ep-subgroupoid of $X$ whose orbit space $S=|\operatorname{supp} \Theta|$ is compact. Then, for every sc-differential $(n-1)$-form $\omega$ on $X_{\infty}$,

$$
\mu_{d \omega}^{(S, \vartheta)}(S)=\mu_{\omega}^{(\partial S, \vartheta)}(\partial S)
$$

Our construction is compatible with equivalences between ep-groupoids as the following theorem shows. 
Theorem 3.27 (Equivalences) Assume that $F: X \rightarrow Y$ is an equivalence between the ep-groupoids $X$ and $Y$. Assume that $\Theta: Y \rightarrow \mathbb{Q}^{+}$is an oriented $n$-dimensional branched ep-subgroupoid of $Y$ whose orbit space $S=|\operatorname{supp} \Theta|$ is compact and equipped with the weight function $\vartheta: S \rightarrow \mathbb{Q}^{+}$defined by $\vartheta(|y|)=\Theta(y)$ for $|y| \in S$. Define the $n$-dimensional branched ep-subgroupoid on $X$ by $\Theta^{\prime}:=\Theta \circ F: X \rightarrow \mathbb{Q}^{+}$ and denote by $S^{\prime}$ and $\vartheta^{\prime}$ the associated orbit space and the weight function on $S^{\prime}$. Moreover, assume that $\Theta^{\prime}$ is equipped with the induced orientation. Then, for every sc-differential $n$-form $\omega$ on $Y_{\infty}$,

$$
\mu_{\omega}^{(S, \vartheta)} \circ|F|=\mu_{\omega^{\prime}}^{\left(S^{\prime}, \vartheta^{\prime}\right)}
$$

where the $n$-form $\omega^{\prime}$ on $X_{\infty}$ is the pull back form $\omega^{\prime}=F^{*} \omega$. Similarly,

$$
\mu_{\tau}^{(\partial S, \vartheta)} \circ|F|=\mu_{\tau^{\prime}}^{\left(\partial S^{\prime}, \vartheta^{\prime}\right)}
$$

for every $(n-1)$-form $\tau$ on $Y_{\infty}$.

Theorem 3.27 allows to rephrase the previous theorems in the polyfold set-up.

An sc-differential form on the topological space $Z$ or $Z^{i}$ is defined via the overhead of the postulated polyfold structures. We define an sc-differential form $\tau$ on the polyfold $Z$ as an equivalence class of triples $(X, \beta, \omega)$ in which the pair $(X, \beta)$ is a polyfold structure on $Z$ and $\omega$ an sc-differential form on $X_{\infty}$. Two such triples $(X, \beta, \omega)$ and $\left(X^{\prime}, \beta^{\prime}, \omega^{\prime}\right)$ are called equivalent, if there is a third ep-groupoid $X^{\prime \prime}$ and two equivalences

$$
X \stackrel{F}{\leftarrow} X^{\prime \prime} \stackrel{F^{\prime}}{\longrightarrow} X^{\prime}
$$

satisfying $\beta \circ|F|=\beta^{\prime} \circ\left|F^{\prime}\right|$ and, in addition,

$$
F^{*} \omega=\left(F^{\prime}\right)^{*} \omega^{\prime}
$$

for the pull back forms on $X^{\prime \prime}$. We shall abbreviate an equivalence class by $\tau=[\omega]$ where $(X, \beta, \omega)$ is a representative of the class and call $\tau$ an sc-differential form on the polyfold $Z$. Again we have a directed system obtained from the inclusions $Z^{i+1} \rightarrow Z^{i}$ which allows us to define, analogously to the ep-groupoid-case, the notion of a differential form on $Z_{\infty}$. We shall still use the symbol $[\omega]$ for such forms. It is important to keep in mind that a representative $\omega$ of $[\omega]$ is an sc-differential form on $X_{\infty}$ where $(X, \beta)$ is a polyfold structure on $Z$.

The exterior derivative of an sc-differential form $[\omega]$ is defined by

$$
d[\omega]=[d \omega] .
$$

Let $S$ be a branched suborbifold $S$ of a polyfold $Z$ equipped with a weight function $w: S \rightarrow \mathbb{Q}^{+} \cap(0, \infty)$ together with an equivalence class of triples $(X, \beta, \Theta)$ in which 
the pair $(X, \beta)$ is a polyfold structure on $Z$ and $\Theta: X \rightarrow \mathbb{Q}^{+}$is an ep-subgroupoid of $X$ satisfying $S=\beta(|\operatorname{supp} \Theta|)$ and $w(\beta(|x|))=\Theta(x)$ for $x \in \operatorname{supp} \Theta$. The "boundary" set $\partial S$ of a branched suborbifold $S$ is defined by setting $\partial S=\beta(|\operatorname{supp} \Theta \cap \partial X|)$ for a representative $(X, \beta, \Theta)$ of the equivalence class. From the previous results it follows that for a compact and oriented branched suborbifold $S \subset Z$ of a polyfold $Z$ there is a canonical $\sigma$-algebra $(S, \mathcal{L}(S))$ of measurable subsets and a well-defined integration theory for which Stokes' theorem holds.

Theorem 3.28 Let $Z$ be a polyfold and $S \subset Z$ be an oriented compact branched suborbifold defined by the equivalence class $[(X, \beta, \Theta)]$ and equipped with the weight function $w: S \rightarrow \mathbb{Q}^{+} \cap(0, \infty)$. For an sc-differential $n$-form $\tau$ on $Z_{\infty}$ and $K \in \mathcal{L}(S)$, we define

$$
\int_{(K, w)} \tau:=\int_{\beta^{-1}(K)} d \mu_{\omega}^{\left(\beta^{-1}(S), \vartheta\right)}=\mu_{\omega}^{\left(\beta^{-1}(S), \vartheta\right)}\left(\beta^{-1}(K)\right),
$$

where the equivalence class $\tau$ is represented by the triple $(X, \beta, \omega)$ and where the weight function $\vartheta$ on $\beta^{-1}(S)=|\operatorname{supp} \Theta|$ is defined by $\vartheta(|x|)=\Theta(x)$. Then the integral $\int_{(K, w)} \tau$ is independent of the representative $(X, \beta, \omega)$ in the equivalence class. Moreover, if $\tau$ is an sc-differential $(n-1)-$ form on $Z_{\infty}$, then

$$
\int_{(\partial S, w)} \tau=\int_{(S, w)} d \tau
$$

For all the details and the proofs of the results of Section 3.3 we refer to [20].

\subsection{Strong polyfold bundles}

In the following we assume that $p: W \rightarrow Z$ is a continuous and surjective map between two paracompact second countable spaces.

Definition 3.29 A strong (polyfold) bundle structure for $p$ consists of a triple $(E, \Gamma, \gamma)$ in which $P: E \rightarrow X$ is a strong bundle over the ep-groupoid $X, \Gamma$ is a homeomorphism between the orbit space $|E|$ and $W$, and $\gamma$ is a homeomorphism from the orbit space $|X|$ to $Z$. Further, we require that

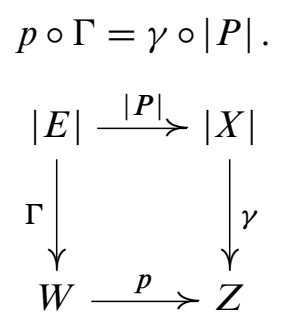


The two triples $(E, \Gamma, \gamma)$ and $\left(E^{\prime}, \Gamma^{\prime}, \gamma^{\prime}\right)$ are called equivalent if there exists an sbundle isomorphism $\mathfrak{A}: E \Rightarrow E^{\prime}$ with the underlying s-isomorphism a: $X \Rightarrow X^{\prime}$ satisfying on the orbit spaces

$$
\Gamma=\Gamma^{\prime} \circ|\mathfrak{A}| \text { and } \gamma=\gamma^{\prime} \circ|\mathfrak{a}| .
$$

We note that the second relation is a consequence of the first relation.

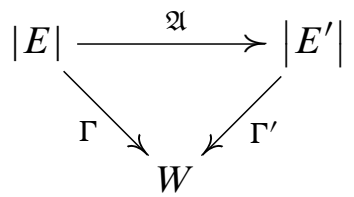

We are in the position to define the notion of a strong polyfold bundle.

Definition 3.30 A strong polyfold bundle $p: W \rightarrow Z$ consists of a surjective continuous map between two second countable paracompact topological spaces together with an equivalence class of strong bundle structures $(E, \Gamma, \gamma)$ for $p$.

Next we introduce the notion of an sc-smooth section of the strong polyfold bundle $p: W \rightarrow Z$. In order to do so, we take a model $(E, \Gamma, \gamma)$ where $P: E \rightarrow X$ is a strong bundle over the ep-groupoid $X$ and consider a pair $(f, F)$ in which $f$ is a continuous section of the bundle $p$ and $F \in \Gamma(P)$ an sc-smooth section of the bundle $P$ satisfying

$$
f \circ \gamma=\Gamma \circ|F|
$$

in diagrams:

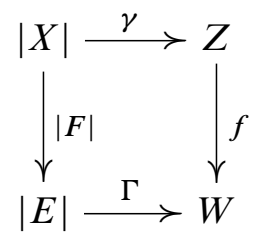

Let $\left(E^{\prime}, \Gamma^{\prime}, \gamma^{\prime}\right)$ be an equivalent strong bundle structure for $p$ where $P^{\prime}: E^{\prime} \rightarrow X^{\prime}$ is a strong bundle over the ep-groupoid $X^{\prime}$. We consider the pair $\left(f^{\prime}, F^{\prime}\right)$ of sections of the bundle $p$ and $F^{\prime} \in \Gamma\left(P^{\prime}\right)$ satisfying $f^{\prime} \circ \gamma^{\prime}=\Gamma^{\prime} \circ\left|F^{\prime}\right|$ and call the two pairs $(f, F)$ and $\left(f^{\prime}, F^{\prime}\right)$ equivalent if $f^{\prime}=f$ and if there exists an s-bundle isomorphism $\mathfrak{A}: E \Rightarrow E^{\prime}$ whose pushforward satisfies

$$
\mathfrak{A}_{*}(F)=F^{\prime} .
$$

Definition 3.31 With the above notation an equivalence class $f=[f, F]$ of sections is called an sc-smooth section of the polyfold bundle $p: W \rightarrow Z$ and the pair $(f, F)$ is called a representative of the map $f$ for the model $P: E \rightarrow X$. The $\mathrm{sc}^{+}-$sections and the Fredholm sections are defined similarly. 
In view of Proposition 2.25, these concepts are well-defined. We will denote the space of sc-smooth sections of the bundle $p: W \rightarrow Z$ by $\Gamma(p)$ and the corresponding space of $\mathrm{sc}^{+}-$sections by $\Gamma^{+}(p)$.

The auxiliary norm for a strong polyfold bundle is defined as follows.

Definition 3.32 An auxiliary norm for the strong polyfold bundle $p: W \rightarrow Z$ consists of a map $N: W_{0,1} \rightarrow[0, \infty)$ having the following property. If the strong bundle $P: E \rightarrow X$ over the ep-groupoid $X$ is a model representing the strong bundle structure for $p$, then

$$
N^{*}: E_{0,1} \rightarrow[0, \infty), \quad e \mapsto N(\Gamma(|e|))
$$

is an auxiliary norm for the bundle $P: E \rightarrow X$. The subset $W_{0,1} \subset W$ is defined by $W_{0,1}=\Gamma\left(\left|E_{0,1}\right|\right)$.

Let us observe that in general $N$ is fiberwise not a norm since the fibers do not have a linear structure. Nevertheless, a local representative $N^{*}$ is an auxiliary norm having the additional property that the existence of a morphism $\varphi: h \rightarrow h^{\prime}$ implies $N^{*}(h)=N^{*}\left(h^{\prime}\right)$. Alternatively, given an auxiliary norm $N^{*}$ for the local model $E \rightarrow X$ satisfying $N^{*}(h)=N^{*}(k)$ if there exists a morphism $h \rightarrow k$, we can define $N(w)$ for $w \in W_{0,1}$ by $N(w)=N^{*}(h)$ if $\Gamma(|h|)=w$. This defines an auxiliary norm for the strong polyfold bundle $p$.

Next we introduce the notion of mixed convergence in $W$.

Definition 3.33 A sequence $\left(w_{k}\right)$ in $W_{0,1}$ is called mixed convergent to $w$ if there exists a local model $(E, \Gamma, \gamma)$ so that the sequence $\left(\left|h_{k}\right|\right):=\left(\Gamma^{-1}\left(w_{k}\right)\right) \subset|E|$ has suitable representatives, say $h_{k}$ of $\left|h_{k}\right|$ and $h$ of $|h|$ so that $h_{k}$ is m-convergent to $h$ on $E_{0,1}$. Let us note that the particular choice of local coordinates in the definition is irrelevant.

For the definition of the m-convergence we refer to [22].

\section{5 $\mathrm{Sc}^{+}-$multisections}

In this section we define $\mathrm{sc}^{+}$-multisections. Again we view the nonnegative rational numbers $\mathbb{Q}^{+}=\mathbb{Q} \cap[0, \infty)$ as a category with the identities as morphisms. The following definition brings a definition in Cieliebak, Mundet i Riera and Salamon [2] into the groupoid framework. 
Definition 3.34 Let $P: E \rightarrow X$ be a strong bundle over the ep-groupoid $X$. Then an $\mathrm{sc}^{+}-$multisection of $P$ is a functor

$$
\Lambda: E \rightarrow \mathbb{Q}^{+}
$$

such that the following local representation (called local section structure) holds true. Every object $x \in X$ possesses an open neighborhood $U_{x}$ on which the isotropy group $G_{X}$ acts by its natural representation $\varphi_{g} \in \operatorname{Diff}_{\mathrm{sc}}\left(U_{x}\right)$ and finitely many $\mathrm{sc}^{+}{ }_{-}$sections $s_{1}, \ldots, s_{k}: U_{x} \rightarrow E$ (called local sections) with associated positive rational numbers $\sigma_{1}, \sigma_{2}, \ldots, \sigma_{k}$ (called weights) satisfying the following properties:

$$
\Lambda(e)=\sum_{j=1}^{k} \sigma_{j}=1,
$$

for all $e \in E \mid U$ for which there exists a section $s_{j}$ satisfying $s_{j}(P(e))=e$. If there is no such section, then $\Lambda(e)=0$.

The functoriality of $\Lambda$ implies $\Lambda\left(e^{\prime}\right)=\Lambda(e)$ if there is a morphism $e^{\prime} \rightarrow e$ in $\mathbf{E}$. Explicitly,

$$
\Lambda(\mu(\varphi, e))=\Lambda(e)
$$

for all $(\varphi, e) \in \mathbf{X}_{s} \times_{p} E$. Hence $\Lambda$ induces the map $|\Lambda|:|E| \rightarrow \mathbb{Q}^{+}$on the orbit space. We shall denote the collection of all sc ${ }^{+}$-multisections of the strong bundle $P: E \rightarrow X$ by $\Gamma_{m}^{+}(P)$. For every $x \in X$, the set

$$
\operatorname{supp}(x):=\{e \in E \mid P(e)=x, \Lambda(e)>0\}
$$

is finite, and $\sum_{e \in \operatorname{supp}(x)} \Lambda(e)=1$. Moreover, if $x \in U$, then

$$
\operatorname{supp}(x)=\left\{s_{1}(x), \ldots, s_{k}(x)\right\},
$$

where $s_{1}, \ldots, s_{k}: U \rightarrow E$ are the local $\mathrm{sc}^{+}$-sections.

Definition 3.35 The $\mathrm{sc}^{+}$-multisection $\Lambda$ is called trivial on the set $V \subset X$ if $\Lambda$ is identically equal to 1 on the zero section over $V$, ie, $\Lambda\left(0_{x}\right)=1$ for all $x \in V$ (and hence $\Lambda\left(e_{x}\right)=0$ for all $\left.e_{x} \neq 0_{x}\right)$.

The support of the $\mathrm{sc}^{+}-$multisection $\Lambda$ is the smallest closed set in $X$ outside of which $\Lambda$ is trivial. 


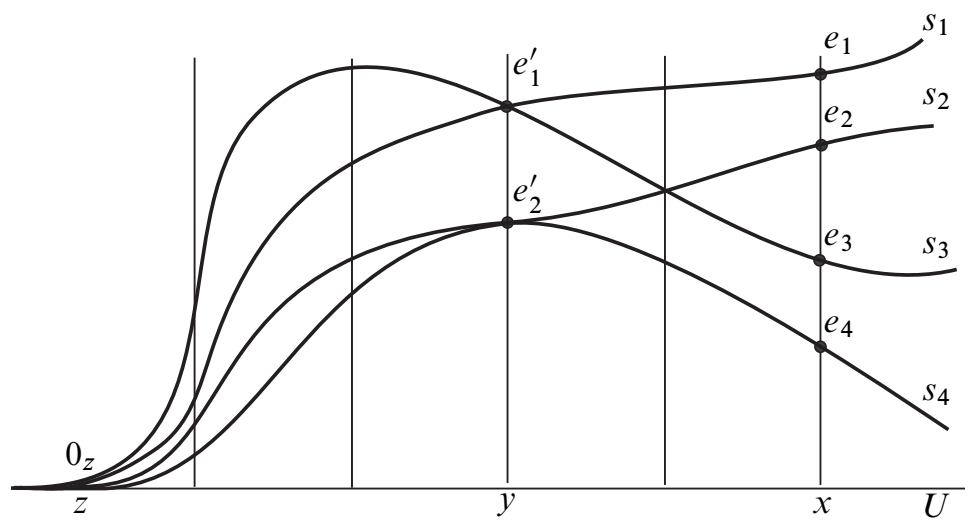

Figure 2: Here $k=4$. If $P\left(e_{j}\right)=x$ for $j=1,2,3,4$, then $\Lambda\left(e_{j}\right)=\sigma_{j}$ while if $P\left(e_{j}^{\prime}\right)=y$ for $j=1,2$, then $\Lambda\left(e_{1}^{\prime}\right)=\sigma_{1}+\sigma_{3}$ and $\Lambda\left(e_{2}^{\prime}\right)=\sigma_{2}+\sigma_{4}$. Moreover, $\Lambda\left(0_{z}\right)=\sum_{j=1}^{4} \sigma_{j}=1$ where $0_{z} \in E$ is the zero vector in the fiber over $z$.

If $\Lambda$ and $\Lambda^{\prime}$ are two multisections in $\Gamma_{m}^{+}(P)$ we define their sum as the multisection

$$
\left(\Lambda \oplus \Lambda^{\prime}\right)(e)=\sum_{e^{\prime}+e^{\prime \prime}=e} \Lambda\left(e^{\prime}\right) \cdot \Lambda^{\prime}\left(e^{\prime \prime}\right) .
$$

Explicitly, if the local section structure of $\Lambda$ is represented by the $\mathrm{sc}^{+}-$sections $s_{1}, \ldots, s_{k}: U \rightarrow E$ having the associated weights $\sigma_{1}, \ldots \sigma_{k}$ and the local section structure of $\Lambda^{\prime}$ by the $\mathrm{sc}^{+}$-sections $s_{1}^{\prime}, \ldots, s_{l}^{\prime}: U \rightarrow E$ with the associated weights $\sigma_{1}^{\prime}, \ldots \sigma_{l}^{\prime}$, then at the vector $e_{x} \in E$ satisfying $P\left(e_{x}\right)=x$,

$$
\left(\Lambda \oplus \Lambda^{\prime}\right)\left(e_{x}\right)=\sum_{s_{i}(x)+s_{j}^{\prime}(x)=e_{x}} \sigma_{i} \cdot \sigma_{j}^{\prime} .
$$

Hence $s_{i}+s_{j}^{\prime}: U \rightarrow E$ are the $\mathrm{sc}^{+}$-sections and $\sigma_{i} \cdot \sigma_{j}^{\prime}$ the associated weights of the multisection $\Lambda \oplus \Lambda^{\prime} \in \Gamma_{m}^{+}(P)$ where $1 \leq i \leq k$ and $1 \leq j \leq l$.

The sum of two $\mathrm{sc}^{+}$-multisections is by definition their associated convolution product. We prefer to call it sum rather than convolution product since in the single-valued case it precisely corresponds to the sum.

If $\Lambda_{0}, \Lambda_{1} \in \Gamma_{m}^{+}(P)$ and $\alpha \in(0,1) \cap \mathbb{Q}^{+}$, we can define the sc ${ }^{+}$-multisection $\Lambda_{\alpha} \in$ $\Gamma_{m}^{+}(P)$ by

$$
\Lambda_{\alpha}(e)=\alpha \Lambda_{1}(e)+(1-\alpha) \Lambda_{0}(e) .
$$

It is locally represented by all the local sections of $\Lambda_{0}$ and $\Lambda_{1}$, together with all the associated weights multiplied by $\alpha$, respectively by $(1-\alpha)$.

We shall make use of an auxiliary norm $N^{*}$ for a strong bundle $P: E \rightarrow X$ over the ep-groupoid $X$. 
The first step in the construction of an $\mathrm{sc}^{+}$-multisection is the following lemma.

Lemma 3.36 Consider the strong bundle $P: E \rightarrow X$ over the ep-groupoid $X$ equipped with a compatible auxiliary norm $N^{*}$ and modeled on separable sc-Hilbert spaces. Then, given a smooth point $x_{0} \in X$, a smooth $h_{0} \in E_{x_{0}}$, and an open neighborhood

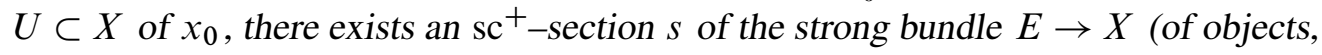
ignoring the morphisms) satisfying

$$
s\left(x_{0}\right)=h_{0}
$$

and having its support in $U$. If $N^{*}(h)<\varepsilon$, we can choose the section $s$ in such a way that

$$
N^{*}(s(y))<\varepsilon \quad \text { for all } y \in X \text {. }
$$

Proof The result is local. We take a strong M-polyfold bundle chart $\Phi: p^{-1}(U) \rightarrow$ $K^{\mathcal{R}}$ covering the sc-diffeomorphism $\varphi: U \rightarrow O$ as defined in [21, Definition 4.8]. The set $O$ is an open subset of the splicing core $K^{\mathcal{S}}=\left\{(v, e) \in V \oplus E^{\prime} \mid \pi_{v}(e)=e\right\}$ associated with the splicing $\mathcal{S}=\left(\pi, E^{\prime}, V\right)$ and $K^{\mathcal{R}}=\left\{((v, e), u) \in O \oplus F \mid \rho_{(v, e)}(u)=\right.$ $u\}$ is the splicing core associated with the strong bundle splicing $\mathcal{R}=(\rho, F,(O, \mathcal{S}))$. In these coordinates the smooth point $x_{0}$ corresponds to the smooth point $\varphi\left(x_{0}\right)=$ $:\left(v_{0}, e_{0}\right) \in O$. Moreover, $\Phi\left(x_{0}, h_{0}\right)=\left(\left(v_{0}, e_{0}\right), h_{0}^{\prime}\right) \in K^{\mathcal{R}}$ where $h_{0}^{\prime}$ is a smooth point in $F$, ie, $h_{0}^{\prime} \in F_{\infty}$. For points $(v, e) \in O$ close to $\left(v_{0}, e_{0}\right)$ we define the local section $s: K^{\mathcal{R}} \rightarrow O$ by

$$
s(v, e)=\left((v, e), \rho_{(v, e)}\left(h_{0}^{\prime}\right)\right) \in K^{\mathcal{R}} .
$$

At the point $\left(v_{0}, e_{0}\right)$ we have $\rho_{\left(v_{0}, e_{0}\right)}\left(h_{0}^{\prime}\right)=h_{0}^{\prime}$ and $s\left(v_{0}, e_{0}\right)=\left(\left(v_{0}, e_{0}\right), h_{0}^{\prime}\right)$ as desired. In view of the definition of a strong bundle splicing, $\rho_{(v, e)}(u) \in F_{m+1}$ if $(v, e) \in O_{m} \oplus F_{m}$ and $u \in F_{m+1}$; see [21, Definition 4.2]. Moreover, the triple $\mathcal{R}^{1}=\left(\rho, F^{1},(O, \mathcal{S})\right)$ is also a general sc-splicing which together with the fact that $h_{0}^{\prime}$ is a smooth point in $F$ implies that the section $s$ of the bundle $K^{\mathcal{R}^{1}} \rightarrow O$ is sc-smooth. Consequently, $s$ is an $\mathrm{sc}^{+}$-section of the local strong M-polyfold bundle $K^{\mathcal{R}} \rightarrow O$. We transport this section by means of the map $\Phi$ to obtain a local sc ${ }^{+}$-section $s$ of the given strong bundle $P: E \rightarrow X$; it satisfies $s\left(x_{0}\right)=h_{0}$. Using Lemma 5.6 in the Appendix (whose proof makes use of the sc-Hilbert structure), we find an sc-smooth bump-function $\beta$ which is equal to 1 near $x_{0}$ so that the section $\beta \cdot s$ has the desired properties.

In general, the section constructed in the proof of Lemma 3.36 will not be compatible with morphisms. Below we shall describe a general recipe for the construction of $\mathrm{sc}^{+}-$ multisections which are compatible with the morphisms. We fix a smooth point $x \in X$ 
and an open neighborhood $U \subset X$ of $x$ which has the distinguished properties listed in the structure Theorem 5.4. In particular, we have the natural representation $\varphi: G_{X} \rightarrow$ $\operatorname{Diff}_{\mathrm{sc}}(U)$ of the isotropy group $G_{x}$ at $x$ and an sc-smooth map $\Gamma: G_{x} \times U \rightarrow \mathbf{X}$ having the following properties:

- $\Gamma(g, x)=g$.

- $s(\Gamma(g, y))=y$ and $t(\Gamma(g, y))=\varphi_{g}(y)$.

- If $h: y \rightarrow z$ is a morphism between $y, z \in U$, then there exists a unique $g \in G_{x}$ with $\Gamma(g, y)=h$.

- Assume that $y_{0} \in X$ is an object for which there exists no morphism $y_{0} \rightarrow x^{\prime}$ for an $x^{\prime}$ in $\bar{U}$. Then there exists an open neighborhood $V$ of $y_{0}$ so that for every $z \in V$ there is no morphism to an element in $\bar{U}$.

- Assume that $y_{0} \in X$ is an object for which there exists no morphism $y_{0} \rightarrow x^{\prime}$ for every $x^{\prime} \in U$, but there exists a morphism to some element in $\bar{U}$. Then, given an open neighborhood $W$ of $\partial U$ (the set theoretic boundary of $U$ ), there exists an open neighborhood $V$ of $y_{0}$ so that if there is a morphism $y \rightarrow x^{\prime}$ for some $y \in V$ and $x^{\prime} \in U$, then $x^{\prime} \in W$.

With the smooth point $x \in U \subset X$ already chosen above we now choose a smooth vector $e_{0} \in E$ satisfying $P\left(e_{0}\right)=x$ and take, using Lemma 3.36, an $\mathrm{sc}^{+}-$section $h$ of the strong bundle $E \rightarrow X$ (of objects) satisfying $h(x)=e_{0}$ and having its support in an open neighborhood $V$ of $x$ which is invariant under the sc-diffeomorphism $\varphi_{g} \in \operatorname{Diff}_{\mathrm{sc}}(U)$ for all $g \in G_{x}$ and whose closure satisfies $\bar{V} \subset U$.

Recall the definition of the strong bundle map $\mu: \mathbf{X}_{s} \times_{p} E \rightarrow E$ from Section 2.4. It acts as follows. If $\varphi: x \rightarrow y$ is a morphism in $\mathbf{X}$, then

$$
\mu(\varphi, \cdot): E_{x} \rightarrow E_{y}
$$

is a linear isomorphism. For every $g \in G_{x}$, we define the $\mathrm{sc}^{+}$-section $h_{g}$ of $E \mid U$ by

$$
h_{g}\left(\varphi_{g}(y)\right):=\mu(\Gamma(g, y), h(y)), \quad y \in U,
$$

and introduce the map

by

$$
\begin{aligned}
& \Lambda_{U}: E \mid U \rightarrow \mathbb{Q}^{+} \\
& \Lambda_{U}(e)=\frac{1}{\sharp G_{X}} \cdot \sharp\left\{g \in G_{X} \mid h_{g}(P(e))=e\right\} .
\end{aligned}
$$

Lemma 3.37 The map $\Lambda_{U}$ defined on $E \mid U$ satisfies $\Lambda_{U}(e)=\Lambda_{U}\left(e^{\prime}\right)$ if there exists a morphism $e \rightarrow e^{\prime}$ in $\mathbf{E}$. 


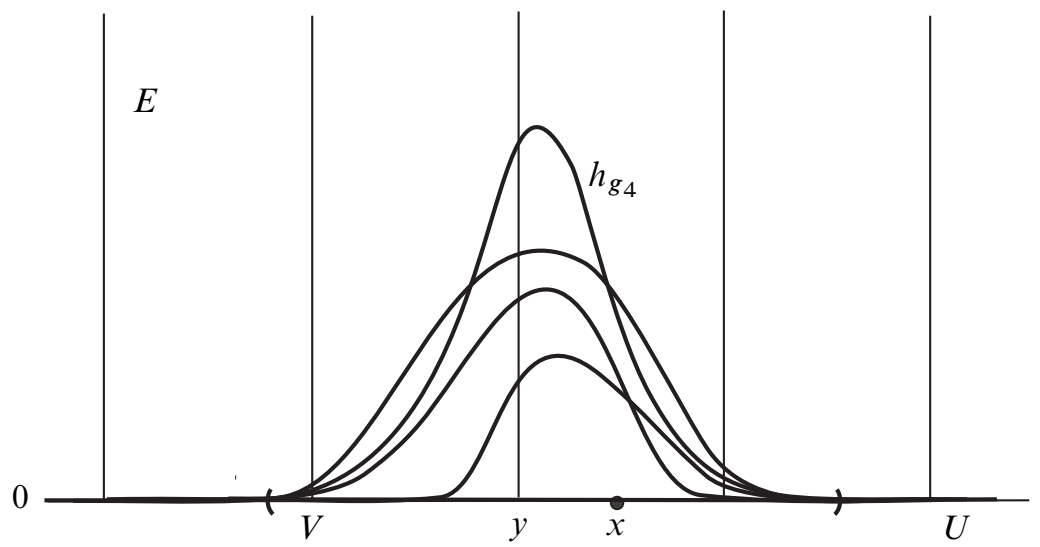

Figure 3: The associated weights are $1 / \sharp G_{x}$

Proof Assume that $e$ and $e^{\prime} \in E \mid U$ are related by a morphism in $\mathbf{E}$. Hence, if $P(e)=y$ and $P\left(e^{\prime}\right)=y^{\prime}$ where $y$ and $y^{\prime} \in U$ so that we can use the notation $e=e_{y}$ and $e^{\prime}=e_{y^{\prime}}$, then there is a morphism $\psi$ in $\mathbf{X}$ satisfying

$$
\psi: y \rightarrow y^{\prime} \text { and } \mu\left(\psi, e_{y}\right)=e_{y^{\prime}} .
$$

By Theorem 2.3 there exists a uniquely determined $g_{0} \in G_{x}$ satisfying $\Gamma\left(g_{0}, y\right)=\psi$ and $\varphi_{g_{0}}(y)=y^{\prime}$. If $g \in G_{x}$ we compute, using the properties of the strong bundle map $\mu: \mathbf{E} \rightarrow E$,

$$
\begin{aligned}
h_{g}\left(\varphi_{g_{0}}(y)\right) & =\mu\left(\Gamma\left(g, \varphi_{g^{-1}}\left(y^{\prime}\right)\right), h\left(\varphi_{g^{-1}}\left(y^{\prime}\right)\right)\right) \\
& =\mu\left(\Gamma\left(g, \varphi_{g^{-1} g_{0}}(y)\right), h\left(\varphi_{g^{-1} g_{0}}(y)\right)\right) \\
& =\mu\left(\Gamma\left(g_{0} g_{0}^{-1} g, \varphi_{g^{-1} g_{0}}(y)\right), h\left(\varphi_{g^{-1} g_{0}}(y)\right)\right) \\
& =\mu\left(\psi \circ \Gamma\left(g_{0}^{-1} g, \varphi_{g^{-1} g_{0}}(y)\right), h\left(\varphi_{g^{-1} g_{0}}(y)\right)\right) \\
& =\mu\left(\psi, \mu\left(\Gamma\left(g_{0}^{-1} g, \varphi_{g^{-1} g_{0}}(y)\right), h\left(\varphi_{g^{-1} g_{0}}(y)\right)\right)\right. \\
& =\mu\left(\Gamma\left(g_{0}, y\right), h_{g_{0}^{-1} g}(y)\right) .
\end{aligned}
$$

Since $\mu(\psi, \cdot): E_{y} \rightarrow E_{y^{\prime}}$ is an isomorphism, it follows that $h_{g_{0}^{-1} g}(y)=e_{y}$ for some $g \in G_{x}$ if and only if $h_{g}\left(\varphi_{g_{0}}(y)\right)=e_{y^{\prime}}$. This implies

$$
\Lambda_{U}(e)=\Lambda_{U}\left(e^{\prime}\right)
$$

The proof of Lemma 3.37 is complete.

Lemma 3.37 shows that the map $\Lambda_{U}: E \mid U \rightarrow \mathbb{Q}^{+}$possesses on $U$ the local section structure defined by the $\mathrm{sc}^{+}$-sections $h_{g}: U \rightarrow E \mid U$ for all $g \in G_{x}$. 
Next we extend the local sc ${ }^{+}$-multisection $\Lambda_{U}$ from $E \mid U$ to all of $E$. In order to first extend $\Lambda_{U}$ from $E \mid U$ to $E \mid \bar{U}$ we recall that the invariant set $V \subset U$ contains the supports of all sections $h_{g}$ for $g \in G_{x}$ and satisfies $\bar{V} \subset U$. Consequently, the $\mathrm{sc}^{+}$-multisection $\Lambda_{U}$ is trivial over the set $U \backslash V$ and, in view of the properties listed in the structure theorem, Theorem 5.4, we can define the multisection $\Lambda$ on the fiber $E_{y}$ over the boundary point $y \in \bar{U} \backslash U$ by $\Lambda(e)=0$ if $e \neq 0_{y}$ and by $\Lambda\left(0_{y}\right)=1$ for the zero $0_{y}$ in $E_{y}$. If $y \notin \bar{U}$ and if there is no morphism $y \rightarrow x^{\prime}$ to some $x^{\prime} \in \bar{U}$ we define $\Lambda$ to be trivial over $y$. On the other hand, if there exists a morphism $e \rightarrow e^{\prime}$ with $P\left(e^{\prime}\right) \in \bar{U}$, we define $\Lambda(e)=\Lambda\left(e^{\prime}\right)$.

So far we have defined a map $\Lambda: E \rightarrow \mathbb{Q}^{+}$and it follows from the construction of $\Lambda$ and from Lemma 3.37 that $\Lambda(e)=\Lambda\left(e^{\prime}\right)$ if there is a morphism $e^{\prime} \rightarrow e$ in $\mathbf{E}$, so that $\Lambda$ induces a functor $\Lambda: E \rightarrow \mathbb{Q}^{+}$.

Proposition 3.38 Let $P: E \rightarrow X$ be a strong bundle over the ep-groupoid $X$. We assume that the sc-smooth structures are based on separable Hilbert spaces. Then the functor $\Lambda: E \rightarrow \mathbb{Q}^{+}$constructed above is an $\mathrm{sc}^{+}-$multisection.

Proof Let $U \subset X$ be the distinguished open set considered above and let $y \in U$. Then there are by construction finitely many local sc ${ }^{+}$-sections, namely $h_{g}$ for $g \in G_{X}$ with associated weights $1 / \sharp G_{x}$, so that $\Lambda$ has the desired local structure. If $y \notin U$ and if there is no morphism $y \rightarrow x^{\prime}$ to some point $x^{\prime} \in \bar{U}$, then $\Lambda$ is trivial over $y$. By the structure theorem, Theorem 5.4, there is an open neighborhood $V$ of $y$ which does not admit morphisms into $\bar{U}$. Consequently, the multisection $\Lambda$ is trivial over the open set $V$. Finally, if $y \notin U$ and if there exists a morphism $y \rightarrow x^{\prime} \in \bar{U}$, then $t \circ s^{-1}$ defines a local diffeomorphism $U(y) \rightarrow U\left(x^{\prime}\right)$ between open neighborhoods so that $\Lambda \mid U(y)$ inherits the local section structure from $\Lambda \mid U\left(x^{\prime}\right)$. This completes the proof of Proposition 3.38 .

We summarize the previous discussion in the following theorem.

Theorem 3.39 Let $P: E \rightarrow X$ be a strong bundle over an ep-groupoid $X$ and let $N^{*}$ be an auxiliary norm for $P$. Assume the sc-smooth structures are based on separable Hilbert spaces. Assume that $e$ is a smooth point in $E$ and $U$ is a saturated open neighborhood in $X$ of the point $x=P(e)$. Then there exists an $\mathrm{sc}^{+}-$multisection $\Lambda: E \rightarrow \mathbb{Q}^{+}$having its support in $U$ (ie, $\Lambda$ is trivial on $X \backslash U$ ) and satisfying $\Lambda(e)>0$. In addition, if $N^{*}(e)<\varepsilon$, then $N^{*}(h)<\varepsilon$ for all $h$ satisfying $\Lambda(h)>0$.

If $\Lambda: E \rightarrow \mathbb{Q}^{+}$is an $\mathrm{sc}^{+}$-multisection for the strong bundle $E \rightarrow X$ and $\Phi: E^{\prime} \rightarrow E$ a strong bundle equivalence covering the equivalence $\varphi: X^{\prime} \rightarrow X$ between the underlying 
ep-groupoids, then the pullback $\Phi^{*} \Lambda: E^{\prime} \rightarrow \mathbb{Q}^{+}$is again an $\mathrm{sc}^{+}$-multisection. If $\Theta$ is an $\mathrm{sc}^{+}$-multisection for $E^{\prime} \rightarrow X^{\prime}$ we can also define the pushforward $\Phi_{*}(\Theta)$ which is an $\mathrm{sc}^{+}$-multisection for the strong bundle $E \rightarrow X$. As in the discussion of sections and generalized isomorphisms we have the following result.

Proposition 3.40 Let $P: E \rightarrow X$ and $P^{\prime}: E^{\prime} \rightarrow X^{\prime}$ be strong bundles over epgroupoids and let $\mathfrak{A}: P \rightarrow P^{\prime}$ be an s-bundle isomorphism. Then $\mathfrak{A}$ induces a bijection

$$
\mathfrak{A}_{*}: \Gamma_{m}^{+}(P) \rightarrow \Gamma_{m}^{+}\left(P^{\prime}\right)
$$

between the corresponding spaces of $\mathrm{sc}^{+}$-multisections. The inverse is the pullback $\mathfrak{A}^{*}$.

The previous construction of the $\mathrm{sc}^{+}-$multisection $\Lambda$ allows also a parametrized version as explained in the following remark, which will be useful in the perturbation theory later on.

Remark (Parametrized $\mathrm{sc}^{+}$-multisections) Take a point $x \in X$ and choose an open neighborhood $U$ of $x$ in the object set of the ep-groupoid $X$. We take the set $U$ so small that the isotropy group $G_{x}$ acts on $U$ by its natural representation. By means of Lemma 3.36, we choose a finite number of $\mathrm{sc}^{+}$-sections $h^{1}, \ldots, h^{k}$ of the strong polyfold bundle $P: E \rightarrow X$ (of objects ignoring the morphisms) having their supports in $U$ and taking at some smooth point $x_{0} \in U$ the prescribed smooth values $e^{j}:=h^{j}\left(x_{0}\right)$. Assuming that $N^{*}\left(e^{j}\right)<\varepsilon / k$, we may achieve, again by Lemma 3.36, that

$$
N^{*}\left(h^{j}(y)\right)<\frac{\varepsilon}{k} \quad \text { for all } 1 \leq j \leq k \text { and all } y \in U .
$$

By $|t|_{\infty}$ we denote the $\ell^{\infty}$-norm of $t=\left(t_{1}, \ldots, t_{k}\right) \in \mathbb{R}^{k}$. For every $t \in \mathbb{R}^{k}$ satisfying $|t|_{\infty}<1$, we define the $\mathrm{sc}^{+}$-section $h_{t}$ as the linear combination

$$
h_{t}(y)=\sum_{j=1}^{k} t_{j} \cdot h^{j}(y) .
$$

The support of the section $h_{t}$ is contained in $U$ and $N^{*}\left(h_{t}(y)\right)<\varepsilon$ for all $y \in X$ and all $|t|_{\infty}<1$. Using the group action by the isotropy group $G_{X}$, we define for every $g \in G_{x}$ the $\mathrm{sc}^{+}-$sections $h_{t, g}$ of $E \mid U$ by

$$
\begin{aligned}
h_{t, g}\left(\varphi_{g}(y)\right) & =\mu\left(\Gamma(g, y), h_{t}(y)\right) \\
& =\sum_{j=1}^{k} t_{j} \cdot \mu\left(\Gamma(g, y), h^{j}(y)\right) .
\end{aligned}
$$


Now proceeding as in Lemma 3.36 and Proposition 3.38, we obtain for every $|t|_{\infty}<1$ an $\mathrm{sc}^{+}$-multisection $\Lambda^{t}: E \rightarrow \mathbb{Q}^{+}$.

In order to construct transversal perturbations of Fredholm sections later on we will make use of the free choice of the smooth images $e^{j}$ at the smooth point $x_{0}$ to fill up the cokernel of the linearized Fredholm section at $x_{0}$.

Multisections for polyfolds will also be defined by means of the overheads as follows. If $p: W \rightarrow Z$ is a strong polyfold bundle, we choose a strong polyfold bundle structure $(E, \Gamma, \gamma)$ for $p$ in which $P: E \rightarrow X$ is a strong bundle over the ep-groupoid $X$ and $\Gamma:|E| \rightarrow W$ the homeomorphism covering the homeomorphism $\gamma:|X| \rightarrow Z$.

Consider a pair $(\lambda, \Lambda)$ in which $\Lambda: E \rightarrow \mathbb{Q}^{+}$is an $\mathrm{sc}^{+}$-multisection on $P$ and $\lambda: W \rightarrow \mathbb{Q}^{+}$is the function satisfying

$$
\lambda(w)=\Lambda(e) \text { if } w=\Gamma(|e|) .
$$

If $\left(E^{\prime}, \Gamma^{\prime}, \gamma^{\prime}\right)$ is a second such model for our strong polyfold bundle $p$ and if $\left(\lambda^{\prime}, \Lambda^{\prime}\right)$ is the corresponding pair, we call the two pairs equivalent if

$$
\lambda=\lambda^{\prime}
$$

and, moreover, if there exists an s-bundle isomorphism $\mathfrak{A}: E \Rightarrow E^{\prime}$ so that for a representative

$$
E \stackrel{\Phi}{\longleftarrow} E^{\prime \prime} \stackrel{\Psi}{\rightarrow} E^{\prime}
$$

(in which $\Phi$ and $\Psi$ are strong bundle equivalences), the $\operatorname{sc}^{+}$-multisections $\Lambda: E \rightarrow \mathbb{Q}^{+}$ and $\Lambda^{\prime}: E^{\prime} \rightarrow \mathbb{Q}^{+}$are related by

$$
\Phi^{*} \Lambda=\Psi^{*} \Lambda^{\prime}
$$

Definition 3.41 $\mathrm{An} \mathrm{sc}^{+}$-multisection for the strong polyfold bundle $p$ : $W \rightarrow Z$ is an equivalence class $[\lambda, \Lambda]$ of pairs.

\section{Global Fredholm theory}

In this section we transplant the basic ideas from the Fredholm theory in [22] to the polyfold set-up. 


\subsection{Fredholm sections}

We begin with the notion of a Fredholm section of a strong polyfold bundle.

Definition 4.1 The section $f$ of the strong polyfold bundle $p: W \rightarrow Z$ is called a Fredholm section provided there exists a representative $F$ of $f$ which is a Fredholm section of the strong bundle $P: E \rightarrow X$ over the ep-groupoid $X$. The latter means that $F: X \rightarrow E$ is an sc-smooth functor and a Fredholm section of the strong bundle $E \rightarrow X$, where $X$ is the M-polyfold of objects, as defined in [22, Definition 3.6].

The Fredholm section $f$ is called proper provided the solution set

$$
S=S(f)=\{z \in Z \mid f(z)=0\}
$$

is compact in $Z$.

Let us observe the following fact, already established in the M-polyfold case.

Proposition 4.2 If $f$ is a proper Fredholm section of the strong polyfold bundle $p: W \rightarrow Z$, then the solution set $S=f^{-1}(0)$ is compact in $Z_{\infty}$.

Proof Let $(E, \Gamma, \gamma)$ be a strong bundle structure for $p$ in which $P: E \rightarrow X$ is a strong bundle over the ep-groupoid $X, \Gamma$ is a homeomorphism between the orbit space $|E|$ and $W$, and $\gamma:|X| \rightarrow Z$ is a homeomorphism. Assume that the section $F$ of the bundle $P: E \rightarrow X$ is a representative of the section $f$. Then $F$ is a proper Fredholm section of the bundle $P$ and, in addition, the section $F$ is a functor. Since $F$ is regularizing the solution set $F^{-1}(0)$ consists of smooth points and therefore $f^{-1}(0)=\gamma\left(\left|F^{-1}(0)\right|\right)$ consists of smooth points in $Z$. Take a sequence $\left(z_{k}\right) \subset f^{-1}(0)$. Since, by assumption, the solution set $S$ is compact in $Z=Z_{0}$, we may assume possibly taking a subsequence that $z_{k} \rightarrow z$ in $Z=Z_{0}$. Choose a point $x \in X$ such that $\pi(x)=|x|=\gamma^{-1}(z)$ where $\pi: X \rightarrow|X|$ is the quotient map. Take an open neighborhood $U$ of $x$ in $X$ which is invariant under the isotropy group $G_{X}$. Then the set $\pi(U)$ is an open neighborhood of $|x|=\gamma(z)$ and $\gamma^{-1}\left(z_{k}\right) \in \pi(U)$ for $k$ large. Since the set $\pi^{-1}(\pi(U))$ consists of points which can be connected by morphisms with points in $U$, we find points $x_{k} \in U$ such that $\pi\left(x_{k}\right)=\left|x_{k}\right|=\gamma^{-1}\left(z_{k}\right)$. Consequently, the sequence $\left(x_{k}\right)$ converges to $x$ in $X_{0}$. Since $F\left(x_{k}\right)=0$ and $x_{k} \rightarrow x$, it follows from the local normal form of a Fredholm section, arguing as in the proof of Theorem 5.11 in [22], that $x_{k} \rightarrow x$ in $X_{\infty}$. Therefore, $z_{k} \rightarrow z$ in $Z_{\infty}$ as claimed. 


\subsection{Properness}

We shall introduce a useful notion for the constructions later on. Assume that $p: W \rightarrow$ $Z$ is a strong polyfold bundle and let $f: Z \rightarrow W$ be a Fredholm section of $p$. We assume further that $N: W_{0,1} \rightarrow[0, \infty)$ is an auxiliary norm and $U$ is an open neighborhood of $f^{-1}(0)$.

Definition 4.3 We say that the pair $(U, N)$ controls compactness if every sequence $\left(z_{k}\right) \subset \bar{U}$ satisfying

$$
\liminf _{k \rightarrow \infty} N\left(f\left(z_{k}\right)\right) \leq 1
$$

possesses a converging subsequence.

Lemma 4.4 Let $P: E \rightarrow X$ be a local model for the strong polyfold bundle $p$ and let $F: X \rightarrow E$ be a Fredholm section representing $f$. Assume that $N^{*}: E_{0,1} \rightarrow[0, \infty)$ is the auxiliary norm for $P$ representing $N$. If the pair $(U, N)$ controls compactness, then the pair $\left(U^{*}, N^{*}\right)$ where $U^{*}=\pi^{-1}(U)$ has the following property. If $\left(x_{k}\right)$ is any sequence in $\overline{U^{*}}$ satisfying

$$
\liminf _{k \rightarrow \infty} N^{*}\left(F\left(x_{k}\right)\right) \leq 1,
$$

then there exist a sequence $\left(y_{k}\right)$ of points in $\overline{U^{*}}$ and a sequence $\left(\varphi_{k}\right)$ of morphisms $\varphi_{k}: x_{k} \rightarrow y_{k}$ so that $\left(y_{k}\right)$ has a converging subsequence.

Proof Without lost of generality we may assume that $Z=|X|$ and $W=|E|$. Take a sequence $\left(x_{k}\right) \subset \overline{U^{*}}$ satisfying $\liminf _{k \rightarrow \infty} N^{*}\left(F\left(x_{k}\right)\right) \leq 1$. Consider the equivalence classes $z_{k}=\left|x_{k}\right|$. Because $x_{k} \in \overline{U^{*}}$, the points $z_{k}$ belong to $\bar{U}$. By definition, $f\left(z_{k}\right)=$ $\left|F\left(x_{k}\right)\right|$ and it follows that $N\left(f\left(z_{k}\right)\right)=N^{*}\left(F\left(x_{k}\right)\right)$ so that $\liminf _{k \rightarrow \infty} N\left(f\left(z_{k}\right)\right) \leq 1$. Since, by assumption, the pair $(U, N)$ controls compactness, it follows that there is a subsequence, again denoted by $\left(z_{k}\right)$, converging to some point $z \in \bar{U}$. Choose a point $x$ such that $z=|x|$. There exists a neighborhood basis $\left(V_{j}\right)_{j \in \mathbb{N}}$ of $x$ such that $V_{j+1} \subset V_{j}$ for all $j \in \mathbb{N}$. Then the sets $\pi\left(V_{j}\right)$ form a decreasing sequence of open neighborhoods of $z$ in $|X|$. Since $z_{k} \rightarrow z$, we find for every $j \in \mathbb{N}$ an index $k_{j}$ such that $z_{k_{j}} \in \pi\left(V_{j}\right)$ and $k_{j+1}>k_{j}$. Hence $x_{k_{j}} \in \pi^{-1}\left(\pi\left(V_{j}\right)\right)$ and since the set $\pi^{-1}\left(\pi\left(V_{j}\right)\right)$ consists of points which are related by morphisms to the points in $V_{j}$, we find for every index $k_{j}$ a point $y_{k_{j}} \in V_{j}$ and a morphism $\varphi_{k_{j}}: x_{k_{j}} \rightarrow y_{k_{j}}$. We note that $y_{k_{j}} \rightarrow x$ as $j \rightarrow \infty$. For $k \neq k_{j}$, we choose $y_{k}=x_{k}$ and take as morphism $\varphi_{k}$ the identity morphism $1_{x_{k}}$. Then $\left(y_{k_{j}}\right)$ is the desired subsequence of the sequence $\left(y_{k}\right)$ converging to $x$. The proof of the lemma is complete. 
As a consequence of the local properness of a Fredholm section [22, Theorem 5.9], we obtain the following result.

Theorem 4.5 Let $p: W \rightarrow Z$ be the strong polyfold bundle with reflexive fibers and let $N$ be an auxiliary norm for $p$. Assume that $f$ is a proper Fredholm section of the bundle $p$. Then there exists an open neighborhood $U$ of the set $S=f^{-1}(0)$ so that the pair $(U, N)$ controls compactness.

Proof We choose a local model $P: E \rightarrow X$ for the strong polyfold bundle $p$ and a proper Fredholm section $F$ of the bundle $P$ representing the section $f$. Without loss of generality we may assume that $Z=|X|$ and $W=|E|$. The map $N^{*}: E_{0,1} \rightarrow[0, \infty)$ defined by $N^{*}(e)=N(|e|)$ is an auxiliary norm for the strong polyfold bundle $P$. The solution set $S=f^{-1}(0) \subset Z$ is, by assumption, compact. Take a point $x \in X$ for which $|x| \in S$. Then $F(x)=0$ and, by the regularizing property of the Fredholm section $F$, the solution $x$ is a smooth point in $X$. In view the local compactness for Fredholm sections of fillable strong M-polyfold bundles, there exists [22, Theorem 5.9] an open neighborhood $U(x) \subset X$ so that every sequence $\left(x_{k}\right)$ in $\overline{U(x)}$ satisfying

$$
\liminf _{k \rightarrow \infty} N^{*}\left(F\left(x_{k}\right)\right) \leq 1
$$

possesses a convergent subsequence. Shrinking $U(x)$ is necessary, we may assume that $U(x)$ is invariant under the action of the isotropy group $G_{x}$. Then the set $|U(x)|$ is open in $Z$. By the compactness of $S$ in $Z$ we find finitely many points $x_{0}, \ldots, x_{K} \in$ $X$ so that $U:=\left|U\left(x_{0}\right)\right| \cup \cdots \cup\left|U\left(x_{K}\right)\right|$ is an open neighborhood of $S$. The set $U^{*}=\pi^{-1}(U)$, where $\pi: X \rightarrow|X|=Z$ is the quotient map onto the orbit space, is an open neighborhood of $F^{-1}(0)$. Let $\left(z_{k}\right)$ be a sequence of points in $\bar{U}$ satisfying

$$
\lim _{k \rightarrow \infty} N\left(f\left(z_{k}\right)\right) \leq 1 .
$$

Then we find a sequence $\left(y_{k}\right) \in \overline{U\left(x_{0}\right)} \cup \cdots \cup \overline{U\left(x_{K}\right)}$ satisfying $\left|y_{k}\right|=z_{k}$. Consequently,

$$
\lim _{k \rightarrow \infty} N^{*}\left(F\left(y_{k}\right)\right) \leq 1
$$

By construction, the sequence $\left(y_{k}\right)$ has a convergent subsequence. Therefore, the sequence $z_{k}=\left|y_{k}\right|$ in the orbit space $|X|=Z$ has a convergent subsequence and the proof of the theorem is complete.

\subsection{Transversality and solution set}

We consider a strong polyfold bundle $p: W \rightarrow Z$ and let $f$ and $\lambda$ be a proper Fredholm section and an $\mathrm{sc}^{+}$-multisection of the bundle $p$, respectively. We denote by 
$P: E \rightarrow X$ the local model of the bundle $p$ and by $F$ the Fredholm section representing $f$ and by $\Lambda: E \rightarrow \mathbb{Q}^{+}$the corresponding $\mathrm{sc}^{+}$-multisection representing $\lambda$. First we define the solution set.

Definition 4.6 The solution set $S:=S(f, \lambda)$ of the pair $(f, \lambda)$ is the set

$$
S(f, \lambda)=\{z \in Z \mid \lambda(f(z))>0\} .
$$

At this point $S$ as a subset of $Z$ is just a second countable paracompact topological space (as a closed subset of $Z$ ). We shall see, however, that in case a certain transversality condition is met, $S$ carries an additional structure so that not only one can talk about orientability of $S$ but also about the integration of sc-differential forms over $S$. Of course, in developing this additional structure, the overhead given by the various representatives $(F, \Lambda)$ describing $(f, \lambda)$ will be important. Taking the representative $(F, \Lambda)$ of $(f, \lambda)$, we consider the solution set

$$
\mathcal{S}=S(F, \Lambda)=\{x \in X \mid \Lambda(F(x))>0\} .
$$

Recall that if $x$ belongs to the solution set, then there exist, in view of the definition of an $\mathrm{sc}^{+}$-multisection, an open neighborhood $U \subset X$ of $x$ and finitely many local $\mathrm{sc}^{+}$-sections $s_{i}: U \rightarrow E$, for $i \in I$, having the associated positive rational weights $\sigma_{i}$, $i \in I$, so that

$$
\sum_{i \in I} \sigma_{i}=1 \quad \text { and } \quad \Lambda(F(x))=\sum_{\left\{j \in I \mid F(x)=s_{j}(x)\right\}} \sigma_{j}
$$

and there is at least one index $j \in I$ such that

$$
F(x)=s_{j}(x) .
$$

If $\Lambda(F(x))=0$, then there is no index $j \in I$ for which $F(x)=s_{j}(x)$.

The natural map

$$
\mathcal{S} \rightarrow S, \quad x \mapsto \gamma(|x|)
$$

induces a homeomorphism $|\mathcal{S}| \rightarrow S$. The solution set $S$ comes with the natural map $\lambda_{f}: S \rightarrow \mathbb{Q}^{+} \cap(0, \infty)$, defined by

$$
\lambda_{f}(z)=\lambda(f(z))
$$

and called the weight function on $S$.

We want to study the pairs $\left(S(f, \lambda), \lambda_{f}\right)$ provided some transversality conditions are met so that the pair $\left(S(f, \lambda), \lambda_{f}\right)$ has the structure of a smooth branched suborbifold with boundary with corners. These transversality conditions are defined in terms of a representative $(F, \Lambda)$ as follows. 
Definition 4.7 Let $p: W \rightarrow Z$ be a strong polyfold bundle and let $(f, \lambda)$ be a pair in which $f$ is a Fredholm section of $p$ and $\lambda: W \rightarrow \mathbb{Q}^{+}$an sc ${ }^{+}$-multisection on $p$. Assume that the strong bundle $P: E \rightarrow X$ over the ep-groupoid $X$ is a model representing $p$ and the Fredholm section $F: X \rightarrow E$ of the bundle $P$ and the $\mathrm{sc}^{+}-$multisection $\Lambda: E \rightarrow \mathbb{Q}^{+}$of $P$ are representatives of $f$ and $\lambda$.

(1) The pair $(f, \lambda)$ is called a transversal pair if for every $z \in S(f, \lambda)$ the following holds. If $x \in X$ represents $z$ and $\left(a_{i}\right)$ is a local section structure for $\Lambda$ near $x$, then for every $i$ for which $F(x)-a_{i}(x)=0$, the linearization $\left(F-a_{i}\right)^{\prime}(x): T_{x} X \rightarrow E_{x}$ is surjective.

(2) The pair $(f, \lambda)$ is in good position if for every $z \in S(f, \lambda)$ the following holds. If $x \in X$ represents $z$ and $\left(a_{i}\right)$ is a local section structure for $\Lambda$ near $x$, then for every $i$ for which $F(x)-a_{i}(x)=0$, the linearization $\left(F-a_{i}\right)^{\prime}(x)$ is surjective and its kernel is in good position to the corner structure of $X$ in the sense of Definition 4.14 in [22].

(3) The pair $(f, \lambda)$ is in general position to the boundary $\partial Z$ if for every $z \in S(f, \lambda)$ the following holds. If $x$ represents $z$ and $\left(a_{i}\right)$ is a local section structure for $\Lambda$ near $x$, then for every $i$ for which $F(x)-a_{i}(x)=0$ the linearization $\left(F-a_{i}\right)^{\prime}(x)$ is surjective and the kernel of $\left(F-a_{i}\right)^{\prime}(x)$ is transversal to $T_{x}^{\partial} X$ in $T_{x} X$. Here $T_{x}^{\partial} X$ is the intersection of all tangent spaces at $x$ to the local faces containing $x$ and we refer to [22] for more details.

Proposition 4.8 If one of the above properties holds true for one local section structure, then it holds true for all the other local sections structures.

To prove the proposition we first introduce the concept of a linearization of a Fredholm section with respect to a multisection. This concept allows a new elegant formulation of Definition 4.7. We consider the Fredholm section $F: X \rightarrow E$ of the strong bundle $p: E \rightarrow X$ and let $\Lambda: E \rightarrow \mathbb{Q}^{+}$be an $\mathrm{sc}^{+}$-multisection. We fix a point $x$ belonging to the solution set $S(F, \Lambda)=\{x \in X \mid \Lambda(F(x))>0\}$ and define the linearization of $F$ at the solution $x$ with respect to $\Lambda$ as follows.

In view of the definition of the $\mathrm{sc}^{+}$-multisection $\Lambda$, there exist an open neighborhood $U_{x}$ of $x$ and a finite collection of local sections $\left(a_{i}\right)_{i \in I}$ with associated weights $\left(\sigma_{i}\right)_{i \in I}$ such that

$$
\Lambda(e)=\sum_{\left\{i \in I \mid a_{i}(P e)=e\right\}} \sigma_{i} .
$$

By $I^{\prime}$ we denote the set of indices $i \in I$ for which $F(x)-a_{i}(x)=0$. If $i, j \in I^{\prime}$, we call the two linearizations $\left(F-a_{i}\right)^{\prime}(x)$ and $\left(F-a_{j}\right)^{\prime}(x): T_{x} X \rightarrow E_{x}$ equivalent if

$$
\left(F-a_{i}\right)^{\prime}(x) \cdot \delta x=\left(F-a_{j}\right)^{\prime}(x) \cdot \delta x \text { for all } \delta x \in T_{x} X .
$$


Denoting by $\left[\left(F-a_{i}\right)^{\prime}(x)\right]$ the equivalence class of the operator $\left(F-a_{i}\right)^{\prime}(x)$, we define the linearization $F_{\Lambda}^{\prime}(x)$ of $F$ at $x$ with respect to the multisection $\Lambda$ to be the finite collection of all equivalence classes

$$
\left[\left(F-a_{i_{1}}\right)^{\prime}(x)\right],\left[\left(F-a_{i_{2}}\right)^{\prime}(x)\right], \ldots,\left[\left(F-a_{i_{n}}\right)^{\prime}(x)\right] .
$$

The notion of the linearization $F_{\Lambda}^{\prime}(x)$ is independent of the choice of the local section structure of the multisection $\Lambda$ as the following proposition, which has Proposition 4.8 as an immediate consequence, shows.

Proposition 4.9 Assume that $\left(a_{i}\right)_{i \in I}$ and $\left(b_{j}\right)_{j \in J}$ are two local section structures for the multisection $\Lambda$ in $U_{x}$ and let

$$
\left[\left(F-a_{i_{1}}\right)^{\prime}(x)\right],\left[\left(F-a_{i_{2}}\right)^{\prime}(x)\right], \ldots,\left[\left(F-a_{i_{n}}\right)^{\prime}(x)\right]
$$

and

$$
\left[\left(F-b_{j_{1}}\right)^{\prime}(x)\right],\left[\left(F-b_{j_{2}}\right)^{\prime}(x)\right], \ldots,\left[\left(F-b_{j_{m}}\right)^{\prime}(x)\right]
$$

be the equivalence classes defined above. Then $n=m$ and for every $i \in\left\{i_{1}, \ldots, i_{n}\right\}$, there exists exactly one $j \in\left\{j_{1}, \ldots, j_{m}\right\}$ such that

$$
\left[\left(F-a_{i}\right)^{\prime}(x)\right]=\left[\left(F-b_{j}\right)^{\prime}(x)\right] .
$$

To prove the proposition we will need the following lemma.

Lemma 4.10 Let $\left(a_{i}\right)_{i \in I}$ and $\left(b_{j}\right)_{j \in J}$ be two local section structures for the multisection $\Lambda$ in the open neighborhood $U_{x} \subset X$ around the solution $x \in S(F, \Lambda)$. Then given $i \in I$ for which $F(x)-a_{i}(x)=0$ and given $\delta x \in T_{x} X$, there exists an index $j \in J$ such that $F(x)-b_{j}(x)=0$ and

$$
\left[F(x)-a_{i}(x)\right]^{\prime} \cdot \delta x=\left[F(x)-b_{j}(x)\right]^{\prime} \cdot \delta x .
$$

Proof Since the smooth tangent vectors are dense on every level, we first consider the case of smooth tangent vectors and deal with the arbitrary tangent vectors later on. We fix an index $i \in I$ for which $F(x)-a_{i}(x)=0$. We work in local coordinates around the solution $x$. Hence we assume that $U=U_{x}$ is a $G_{x}$-invariant open set of the splicing core $K^{\mathcal{S}}=\{(v, e) \in V \oplus G \mid \pi(v, e)=e\}$ associated with the splicing $\mathcal{S}=(\pi, V, G)$ in which $V$ is an open subset of the partial quadrant $C$ of the sc-Banach space $W=\mathbb{R}^{n} \oplus Q$ for some $n$, and $G$ is an sc-Banach space. We introduce the notation $x=\left(v_{0}, e_{0}\right)$ and define for $y=(v, e) \in U_{x} \subset V \oplus G$ the map $A: U_{x} \rightarrow E$ by

$$
A(y)=F(y)-a_{i}(y) .
$$

If $\delta x=(\delta v, \delta e) \in W \oplus G$ is a smooth tangent vector in $T_{x} U$, then

$$
\left(D_{v} \pi\right)(x) \cdot \delta v+\pi_{v_{0}}(\delta e)=\delta e .
$$


Therefore, for small $t$,

$$
\begin{aligned}
& \pi_{v_{0}+t \delta v}\left(e_{0}+t \delta e\right) \\
& =\pi_{v_{0}}\left(e_{0}\right)+t \cdot D \pi\left(v_{0}, e_{0}\right)[\delta v, \delta e]+o(t) \\
& =e_{0}+t \cdot\left[\left(D_{v} \pi\right)\left(v_{0}, e_{0}\right) \cdot \delta v+\pi_{v_{0}}(\delta e]+o(t)\right. \\
& =e_{0}+t \delta e+o(t),
\end{aligned}
$$

where $o(t) / t \rightarrow 0$ as $t \rightarrow 0$ on every level of the sc-Banach space $G$. Consequently, applying the linear projection $\pi_{v_{0}+\delta v}$ to both sides,

$$
\begin{aligned}
\pi_{v_{0}+t \delta v}\left(e_{0}+t \delta e+o(t)\right) & =\left(\pi_{v_{0}+t \delta v}\right)^{2}\left(e_{0}+t \delta e\right) \\
& =\pi_{v_{0}+t \delta v}\left(e_{0}+t \delta e\right) \\
& =e_{0}+t \delta e+o(t) .
\end{aligned}
$$

This implies that the curve $t \mapsto\left(v_{0}+t \delta v, e_{0}+t \delta e+o(t)\right)$ through $x=\left(v_{0}, e_{0}\right)$ belongs to $U_{x} \subset K^{\mathcal{S}}$ for small values of $t$. Let $\left(t_{n}\right)$ be any sequence converging to 0 . We define the sequence of points $x_{n} \in U_{x}$ by

$$
x_{n}=\left(v_{0}+t_{n} \delta v, e_{0}+t_{n} \delta e+o\left(t_{n}\right)\right) .
$$

Then $x_{n} \rightarrow x=\left(v_{0}, e_{0}\right)$ as $n \rightarrow \infty$. Since the local system sections $\left(a_{i}\right)_{i \in I}$ and $\left(b_{j}\right)_{j \in J}$ define the multisection $\Lambda$, it follows that

$$
\Lambda\left(a_{i}\left(x_{n}\right)\right)=\sum_{\left\{j \in J \mid b_{j}\left(x_{n}\right)=a_{i}\left(x_{n}\right)\right\}} \tau_{j}>0
$$

for every $n$. Therefore, there exists a sequence $\left(j_{n}\right) \in J$ of indices such that

$$
b_{j_{n}}\left(x_{n}\right)=a_{i}\left(x_{n}\right) .
$$

Because $J$ is a finite set, there must exist an index $j \in I$ and a subsequence of $\left(x_{n}\right)$ (denoted again by $\left(x_{n}\right)$ ) such that for all $n$

$$
b_{j}\left(x_{n}\right)=a_{i}\left(x_{n}\right) .
$$

As $n \rightarrow \infty$, it follows that

$$
b_{j}(x)=a_{i}(x) .
$$

Introducing the map $B: U_{x} \rightarrow E$ by

$$
B(y)=F(y)-b_{j}(y)
$$

we have proved

$$
A(x)=B(x)=0 \quad \text { and } \quad A\left(x_{n}\right)=B\left(x_{n}\right)
$$

for all $n$. 
Hence, in view of $A\left(x_{n}\right)=A(x)+D A(x) \cdot\left(x_{n}-x\right)+o\left(x_{n}-x\right)$ and $B\left(x_{n}\right)=$ $B(x)+D B(x) \cdot\left(x_{n}-x\right)+o\left(x_{n}-x\right)$, we conclude that

$$
[D A(x)-D B(x)] \cdot\left(x_{n}-x\right)=o\left(x_{n}-x\right)
$$

on every level. Dividing by $t_{n}$ and taking the limit as $n \rightarrow \infty$ we find for the tangent vector $\delta x$,

$$
D A(x) \cdot \delta x=D B(x) \cdot \delta x .
$$

If $\delta x \in T_{x} X$ is an arbitrary tangent vector, we take a sequence $\left(\delta x^{n}\right)$ of smooth tangent vectors converging to $\delta x$. By the first part of the proof, there exists a sequence $\left(j_{n}\right) \subset J$ such that

$$
D\left[F-a_{i}\right](x) \cdot \delta x^{n}=D\left[F-b_{j_{n}}\right](x) \cdot \delta x^{n} .
$$

Because $J$ is a finite set, there exist an index $j$ and a subsequence, again denoted by $\left(\delta x^{n}\right)$, such that

$$
D\left[F-a_{i}\right](x) \cdot \delta x^{n}=D\left[F-b_{j}\right](x) \cdot \delta x^{n}
$$

for all $n$. Taking the limit as $n \rightarrow \infty$, we conclude

$$
D A(x) \cdot \delta x=D B(x) \cdot \delta x .
$$

This completes the proof of Lemma 4.10 .

Proof of Proposition 4.9 We abbreviate by $I^{\prime}$ the set of indices $i \in I$ for which $F(x)-a_{i}(x)=0$ and by $J^{\prime}$ the set of indices $j \in J$ for which $F(x)-b_{j}(x)=0$. To prove the proposition it suffices to show that for given $i \in I^{\prime}$ there exists $j \in J^{\prime}$ such that $\left(F-a_{i}\right)^{\prime}(x) \cdot \delta x=\left(F-b_{j}\right)^{\prime}(x) \cdot \delta x$ for all $\delta x \in T_{x} X$. If this is not the case, then for every $j \in J^{\prime}$, there exists a vector $\delta x^{j} \in T_{x} X$ such that $\left(F-a_{i}\right)^{\prime}(x) \cdot \delta x^{j} \neq\left(F-b_{j}\right)^{\prime}(x) \cdot \delta x^{j}$ for all $j \in J^{\prime}$. From

$$
a_{i}^{\prime}(x)-b_{j}^{\prime}(x)=\left(F-b_{j}\right)^{\prime}(x)-\left(F-a_{i}\right)^{\prime}(x),
$$

if follows that the kernels ker $\left(a_{i}^{\prime}(x)-b_{j}^{\prime}(x)\right)$ are closed proper subspaces of $T_{x} X$. Consequently, applying the Baire category theorem, we conclude

$$
T_{x} X \backslash \bigcup_{j \in J^{\prime}} \operatorname{ker}\left(a_{i}^{\prime}(x)-b_{j}^{\prime}(x)\right) \neq \varnothing .
$$

Hence, in view of (8),

$$
\left(F-a_{i}\right)^{\prime}(x) \cdot \delta x \neq\left(F-b_{j}\right)^{\prime}(x) \cdot \delta x
$$

for every $\delta x \in T_{x} X \backslash \bigcup_{j \in J^{\prime}} \operatorname{ker}\left(a_{i}^{\prime}(x)-b_{j}^{\prime}(x)\right)$ and every $j \in J^{\prime}$. But by Lemma 4.10, given $\delta x \in T_{x} X \backslash \bigcup_{j \in J^{\prime}} \operatorname{ker}\left(F-b_{j}\right)^{\prime}(x)$, we find some $j \in J^{\prime}$ such that 
$\left(F-a_{i}\right)^{\prime}(x) \cdot \delta x=\left(F-b_{j}\right)^{\prime}(x) \cdot \delta x$, contradicting (9). Consequently, for given $i \in I^{\prime}$, there exists an index $j \in J^{\prime}$ such that $\left[\left(F-a_{i}\right)^{\prime}(x)\right]=\left[\left(F-b_{j}\right)^{\prime}(x)\right]$. By the same token, for given $j \in J^{\prime}$, there exists an index $i \in I$ such that $\left[\left(F-b_{j}\right)^{\prime}(x)\right]=\left[\left(F-a_{i}\right)^{\prime}(x)\right]$. It follows that the number of equivalence classes is the same, that is, $n=m$. The proof of Proposition 4.9 is complete.

Definition 4.11 The linearization $F_{\Lambda}^{\prime}(x)$ at the solution $x$ with respect to the multisection $\Lambda$ is called surjective, in good position, or in general position, if the representatives of each of the equivalence classes

$$
\left[\left(F-a_{i_{1}}\right)^{\prime}(x)\right],\left[\left(F-a_{i_{2}}\right)^{\prime}(x)\right], \ldots,\left[\left(F-a_{i_{n}}\right)^{\prime}(x)\right] .
$$

are surjective, in good position, or in general position.

In view of Proposition 4.9, we can reformulate Definition 4.7 independently of the choice of the local section structure of the $\mathrm{sc}^{+}$-multisection $\lambda$ as follows.

Definition 4.12 Let $p: W \rightarrow Z$ be a strong polyfold bundle and let $(f, \lambda)$ be a pair in which $f$ is a Fredholm section of $p$ and $\lambda: W \rightarrow \mathbb{Q}^{+}$an $\mathrm{sc}^{+}$-multisection on $p$. Assume that the strong bundle $P: E \rightarrow X$ over the ep-groupoid $X$ is a model representing the bundle $p$ and let the Fredholm section $F: X \rightarrow E$ of the bundle $P$ and the $\mathrm{sc}^{+}$-multisection $\Lambda: E \rightarrow \mathbb{Q}^{+}$of $P$ be representatives of $f$ and $\lambda$.

(1) The pair $(f, \lambda)$ is called a transversal pair if for every $x$ satisfying $\Lambda(F(x))>0$, the linearization $F_{\Lambda}^{\prime}(x)$ is surjective.

(2) The pair $(f, \lambda)$ is in good position if for every $x$ satisfying $\Lambda(F(x))>0$, the linearization $F_{\Lambda}^{\prime}(x)$ is surjective and in good position to the corner structure of $X$.

(3) The pair $(f, \lambda)$ is in general position to the boundary $\partial Z$ if for every $x$ satisfying $\Lambda(F(x))>0$, the linearization $F_{\Lambda}^{\prime}(x)$ is surjective and in general position to the boundary $\partial X$.

We note that the actual choice of the representing pair $(F, \Lambda)$ in the previous definition is irrelevant. As a consequence of Lemma 5.19 in [22], the condition that the pair $(f, \lambda)$ is in general position to the boundary implies that the pair $(f, \lambda)$ is in good position. The condition of being in good position is very important and we refer to [22] for a comprehensive discussion.

Theorem 4.13 Let $f$ be a proper Fredholm section of the strong polyfold bundle $p: W \rightarrow Z$ without boundary. Assume that the pair $(f, \lambda)$ is transversal and that the solution set $S=S(f, \lambda)=\{z \in Z \mid \lambda(f(z))>0\}$ is compact. Then the pair $\left(S, \lambda_{f}\right)$ carries in a natural way the structure of a compact branched suborbifold of $Z$ without boundary. If $f$ is oriented, then the branched suborbifold $S$ is oriented. 
The theorem is proved along the lines of the next theorem using [22, Theorem 5.14]. In the case with boundary we have the following result.

Theorem 4.14 Let $f$ be a proper Fredholm section of the strong polyfold bundle $p: W \rightarrow Z$ with boundary with corners. Assume that the pair $(f, \lambda)$ is in general position and the solution set $S=S(f, \lambda)=\{z \in Z \mid \lambda(f(z))>0\}$ is compact. Then the pair $\left(S, \lambda_{f}\right)$ carries in a natural way the structure of a compact branched suborbifold of $Z$ with boundary with corners. If $f$ is oriented, then the branched suborbifold $\left(S, \lambda_{f}\right)$ is oriented.

Proof Let $(E, \Gamma, \gamma)$ be a strong bundle structure for $p$ in which $P: E \rightarrow X$ is a strong bundle over the ep-groupoid $X$ and $\Gamma:|E| \rightarrow W$ is the homeomorphism covering the homeomorphism $\gamma:|X| \rightarrow Z$. We choose a Fredholm section $F$ of the bundle $P$ representing the section $f$ and an $\operatorname{sc}^{+}$-multisection $\Lambda: E \rightarrow \mathbb{Q}^{+}$of the bundle $P$ representing the $\mathrm{sc}^{+}-$multisection $\lambda$ of the strong polyfold bundle $p$. The $\mathrm{sc}^{+}-$multisections $\lambda$ and $\Lambda$ are related as follows:

$$
\lambda(w)=\Lambda(e) \text { if } w=\Gamma(|e|) .
$$

We define the functor $\Theta: X \rightarrow \mathbb{Q}^{+}$by

$$
\Theta(x)=\Lambda(F(x)) \quad \text { for } x \in X
$$

and recall that $\lambda_{f}(z)=\lambda(f(z))$ for $z \in Z$. We claim that $\Theta: X \rightarrow \mathbb{Q}^{+}$is a branched ep-subgroupoid of the ep-groupoid $X$ with boundary with corners.

To see this we take $x \in X$ such that $\Lambda(F(x))>0$. In view of the definition of an $\mathrm{sc}^{+}$-multisection, there exist an open neighborhood $U$ of $x$ in $X$, finitely many local $\mathrm{sc}^{+}-$sections $s_{i}$ for $i \in I$, and positive rational weights $\left(\sigma_{i}\right)_{i \in I}$ so that

$$
\sum_{j=1}^{k} \sigma_{j}=1 \text { and } \Lambda(F(x))=\sum_{\left\{j \mid F(x)=s_{j}(x)\right\}} \sigma_{j} .
$$

The sum over the empty set is equal to 0 . Since $\Lambda(F(x))>0$ there exists at least one index $i \in I$ such that $F(x)=s_{i}(x)$. In view of the regularizing property of $F$ and the fact that $s_{i}$ is an $\mathrm{sc}^{+}-$section, it follows that the point $x$ is smooth. Hence the support of $\Theta$, defined by $\operatorname{supp} \Theta=\{x \in X \mid \Theta(x)>0\}$, is contained in $X_{\infty}$. By our assumption, for every $i \in I$ for which the point $x$ solves $F(x)=s_{i}(x)$, the linearization $\left(F-s_{i}\right)^{\prime}(x)$ is in general position to the boundary $\partial X$. Consequently, in view of Theorem 5.18 in [22], the solution set

$$
M_{i}:=\left\{y \in U \mid\left(F-s_{i}\right)(y)=0\right\}
$$


is a smooth manifold with boundary with corners. If $F(x)-s_{i}(x) \neq 0$, then we set $M_{i}=\varnothing$. Hence

$$
\operatorname{supp} \Theta \cap U=\bigcup_{i \in I} M_{i}
$$

In addition,

$$
\Theta(y)=\Lambda(F(y))=\sum_{\left\{i \in I \mid F(y)=s_{i}(y)\right\}} \sigma_{i}=\sum_{\left\{i \in I \mid y \in M_{i}\right\}} \sigma_{i}
$$

for every $y \in U$. Since $U$ can also be taken so that the isotropy group $G_{x}$ acts on $U$ by sc-diffeomorphisms of $U$, we have proved our claim, that $\Theta=\Lambda \circ F: X \rightarrow \mathbb{Q}^{+}$ is a branched ep-subgroupoid of the ep-groupoid $X$.

Let $z \in S$, ie, $\lambda(f(z))>0$. Since, by the definition of the overhead, $f(z)=$ $\Gamma \circ|F| \circ \gamma^{-1}(z)=\Gamma \circ|F|(|x|)=\Gamma(|F(x)|)$, it follows that $\lambda(f(z))=\Lambda(F(x))=$ $\Theta(x)$, where $z=\gamma(x)$. Hence, $x \in X_{\infty}$. We conclude that $S=\gamma(|\operatorname{supp} \Theta|)$ and that if $z=\gamma(|x|)$, then $\lambda_{f}(z)=\Lambda(F(x))=\Theta(x)$.

For orientation questions we make use of the results in [17]. The relevant facts are briefly summarized in the Appendix, Section 5.4. Recall that an orientation for $f$ is given by an orientation of the determinant bundle $\operatorname{DET}(F) \rightarrow X_{\infty}$ defined by the projection map $\operatorname{DET}(F, x) \mapsto x$ introduced in the Appendix, Section 5.4. In contrast to the usual definition of a determinant bundle $\operatorname{det}(F) \rightarrow X$ the fiber over a smooth point $x$ consists of a convex family of linear sc-Fredholm operators which differ by $\mathrm{sc}^{+}$-operators and are obtained as linearizations (These are not unique except at solutions!). Since locally the multisection is represented by $\mathrm{sc}^{+}-$sections we conclude from [17] that the local solution sets have natural orientations compatible with the morphisms. This completes the proof of Theorem 4.14 .

Next we shall prove parts of Theorem 1.4. We consider a strong polyfold bundle $p: W \rightarrow Z$ and two pairs $\left(f_{0}, \lambda_{0}\right)$ and $\left(f_{1}, \lambda_{1}\right)$ in general position where $f_{j}: Z \rightarrow W$ are proper oriented Fredholm sections and where $\lambda_{j}: W \rightarrow \mathbb{Q}^{+}$are $\mathrm{sc}^{+}$-multisections of $p$. Abbreviating by $\pi:[0,1] \times Z \rightarrow Z$ the projection onto the second factor we denote by $\pi^{*}(p)$ the strong polyfold pullback bundle over $[0,1] \times Z$. Let $t \mapsto \lambda_{t}$ be an sc-smooth homotopy of $\mathrm{sc}^{+}$-multisections of the bundle $p$ connecting the $\mathrm{sc}^{+}-$ multisection $\lambda_{0}$ with $\lambda_{1}$. This means that $\hat{\lambda}(t, z)=\lambda_{t}(z)$ for $(t, z) \in[0,1] \times W$ defines an $\mathrm{sc}^{+}$-multisection on $[0,1] \times W$. If $t \mapsto f_{t}$ is an sc-smooth oriented homotopy of Fredholm sections connecting $f_{0}$ with $f_{1}$, then we consider the pair $(\hat{f}, \hat{\lambda})$ consisting 
of the Fredholm section $\hat{f}$ and the $\mathrm{sc}^{+}$-multisection $\hat{\lambda}$ of $\pi^{*}(p)$ defined by

$$
\begin{array}{ll}
\widehat{f}(t, z)=f_{t}(z) & \text { for }(t, z) \in[0,1] \times Z \\
\widehat{\lambda}(t, z)=\lambda_{t}(z) & \text { for }(t, z) \in[0,1] \times W .
\end{array}
$$

Now we assume that the pair $(\hat{f}, \hat{\lambda})$ is in general position and that the solution set $S=\{(t, z) \in[0,1] \times Z \mid \hat{\lambda}(t, \hat{f}(t, z))>0\}$ is compact. Then by Theorem 4.14 the solution set carries the structure of an oriented compact branched suborbifold of $[0,1] \times Z$ whose boundary is in good position to $\partial([0,1] \times Z)$. Introducing the weight function $w(t, z)=\hat{\lambda}(t, \hat{f}(t, z))$ on $S$, the pair $(S, w)$ contains two obvious boundary pieces, namely $\left(S_{1}, w_{1}\right)$ associated with the pair $\left(f_{1}, \lambda_{1}\right)$ and $-\left(S_{0}, w_{0}\right)$ associated with $\left(f_{0}, \lambda_{0}\right)$. Here we have to take the minus sign if we equip $\left(S_{0}, w_{0}\right)$ with the orientation coming from $\left(f_{0}, \lambda_{0}\right)$ by using the obvious orientation convention for the family $f_{t}$ as explained in [17]. These two pieces can be identified as part of the solution space contained in the two faces of $[0,1] \times Z$ defined by $\{i\} \times Z$ for $i=0,1$. There is another boundary piece $\partial S$ which lies in faces of the form $[0,1] \times($ face in $Z$ ) intersecting $S_{0}$ and $S_{1}$ only in points of degeneracy at least 2 (with respect to the degeneracy index of the polyfold $[0,1] \times Z)$ and which is denoted by $(\hat{\partial} S, w)$. The boundary $(\partial S, w)$ has a natural orientation, it is a branched suborbifold only after having removed the points of degeneracy at least 2 which is a closed set of measure 0 .

If the pair $(\omega, \vartheta)$ represents a cohomology class in $H_{\mathrm{dR}}^{*}(Z, \partial Z)$ and $j: \partial Z \rightarrow Z$ is the inclusion map, then

$$
d \omega=0 \quad \text { and } \quad j^{*} \omega=d \theta .
$$

Lemma 4.15 For a pair $(f, \lambda)$ in general position in which $f$ is an proper oriented Fredholm section, the integration map

$$
[\omega, \vartheta] \rightarrow \int_{(S, w)} \omega-\int_{(\partial S, w)} \vartheta
$$

defines a linear map on the deRham cohomology group $H_{\mathrm{dR}}^{*}(Z, \partial Z)$ which is an invariant under nice homotopies.

Proof We compute, assuming that $\omega$ is of degree $n$ and $S_{0}$ and $S_{1}$ are of dimension $n$,

$$
\begin{aligned}
& {\left[\int_{\left(S_{1}, w_{1}\right)} \omega-\int_{\left(\partial S_{1}, w_{1}\right)} \theta\right]-\left[\int_{\left(S_{0}, w_{0}\right)} \omega-\int_{\left(\partial S_{0}, w_{0}\right)} \theta\right]} \\
& \quad=\int_{\left(S_{1}, w_{1}\right)} \omega-\int_{\left(S_{0}, w_{0}\right)} \omega+\int_{(\hat{\partial} S, w)} \omega-\int_{\left(\partial S_{1}, w_{1}\right)} \vartheta+\int_{\left(\partial S_{0}, w_{0}\right)} \vartheta-\int_{(\hat{\partial} S, w)} \omega
\end{aligned}
$$




$$
\begin{aligned}
& =\int_{(\partial S, w)} \omega-\int_{\left(\partial S_{1}, w_{1}\right)} \vartheta+\int_{\left(\partial S_{0}, w_{0}\right)} \vartheta-\int_{(\hat{\partial} S, w)} \omega \\
& =\int_{(S, w)} d \omega-\int_{\left(S_{1}, w_{1}\right)} d \vartheta+\int_{\left(S_{0}, w_{0}\right)} d \vartheta-\int_{(\widehat{\partial} S, w)} \omega \\
& =-\int_{\left(S_{1}, w_{1}\right)} d \vartheta+\int_{\left(S_{0}, w_{0}\right)} d \vartheta-\int_{(\widehat{\partial} S, w)} d \vartheta \\
& =-\int_{(\partial S, w)} d \vartheta=0 .
\end{aligned}
$$

The last integral vanishes since integration of a global sc-form over the boundary $(\partial S, w)$ is like integration over a closed manifold, so that in particular the integral of an exact form vanishes.

The perturbation theory below guarantees such nice homotopies referred to in the lemma, and will then complete the proof of Theorem 1.4.

\subsection{Perturbation results}

In this section we shall adapt the techniques introduced for M-polyfolds in [22] to the functorial setting and show how given sections can be made transversal by small perturbations.

We first recall the kind of problems studied in [22]. In there we consider a proper Fredholm section of the strong M-polyfold bundle $P: E \rightarrow X$ and want to bring the compact set of solutions of $F(x)=0$ into a general position by a small perturbation section $s$, ie, we study the solution set of $F(x)-s(x)=0$. To do so one first constructs finitely many $\mathrm{sc}^{+}$-sections $s_{j}, j=1, \ldots, k$, so that they fill up the cokernel of the linearizations $F^{\prime}(x)$ at all solutions $x$ of $F(x)=0$ and then considers the parametrized proper Fredholm section $F(t, x)=F(x)+\sum_{j=1}^{k} t_{j} s_{j}(x)$ for $(t, x) \in \mathbb{R}^{k} \times X$ with small parameters $t=\left(t_{1}, \ldots, t_{k}\right)$. It has the property that its linearizations $F^{\prime}(0, x)$ at $t=0$ and the solutions $x \in X$ of $F(x)=0$ are surjective. Assuming that $\partial X=\varnothing$ one concludes that the set $M=\left\{(t, y) \in \mathbb{R}^{k} \times X \mid F(t, x)=0\right.$ and $|t|$ sufficiently small $\}$ of the parametrized Fredholm section is a smooth manifold. Then the regular values of the projection $M \rightarrow \mathbb{R}^{k}$ given by $(t, y) \mapsto t$ give the parameter value $t^{*}=\left(t_{1}^{*}, \ldots, t_{k}^{*}\right)$ near $t=0$ for which the perturbation $s=\sum_{j=1}^{k} t_{j}^{*} s_{j}$ has the desired properties in order to conclude that the solution set $\left\{x \in X \mid F\left(t^{*}, x\right)=0\right\}$ of the perturbed problem is a compact smooth manifold.

In the case of $\partial X \neq \varnothing$, one has to add enough $\mathrm{sc}^{+}-$sections so that also the kernels of the linearizations $F^{\prime}(x)$ at the solutions $x$ of $F(x)=0$ are in good position to the 
boundary $\partial X$. Then the corresponding solution set $M$ of the parametrized Fredholm section for small parameter values is a smooth manifold with boundaries with corners. Again one looks at the projection map $M \rightarrow \mathbb{R}^{k}$ defined by $(t, y) \mapsto t$. This map can be restricted to the local faces (which are smooth manifolds with boundaries with corners) and one finds small regular values $t^{*}$ for a finite number of problems. For these parameter values the solution set $\left\{x \in X \mid F\left(t^{*}, x\right)=0\right\}$ of the perturbed problem is in general position to the boundary $\partial X$ and is, therefore, a compact manifold with boundaries with corners.

In the multivalued case we shall have to consider locally a finite number of problems of the kind just described and, using a compactness and a covering argument, in total a finite number of such problems. This way we shall obtain finitely many finite dimensional submanifolds (perhaps with boundary with corners) and look again at the projection map $(t, y) \mapsto t$. A common regular value $t^{*}$ which exists by Sard's theorem gives rise to an $\mathrm{sc}^{+}$-multisection $\Lambda^{t^{*}}$, so that the pair $\left(F, \Lambda^{t^{*}}\right)$ is in general position and gives rise to the branched ep-subgroupoid $\Lambda^{t^{*}} \circ F: X \rightarrow \mathbb{Q}^{+}$of the ep-groupoid $X$.

Now we assume that $\lambda$ is an $\mathrm{sc}^{+}$-multisection on the strong bundle $p$ : $W \rightarrow Z$ and let $N: W_{0,1} \rightarrow[0, \infty)$ be an auxiliary norm for $p$. We choose an $\mathrm{sc}^{+}-$multisection $\Lambda: E \rightarrow \mathbb{Q}^{+}$on the strong bundle $P: E \rightarrow X$ representing $\lambda$ and let $N^{*}: E_{0,1} \rightarrow$ $[0, \infty)$ be an auxiliary norm for $P$ representing $N$. Then we define the auxiliary norm $N(\lambda)$ of the multisection $\lambda$ as follows.

We start by defining the auxiliary norm $N^{*}(\Lambda)$ of the $\mathrm{sc}^{+}$-multisection $\Lambda$. For every $x \in X$, there exists an open neighborhood $U_{x}$ of $x$ on which there is a local section structure $\left(s_{i}\right)_{i \in I}$ consisting of $\mathrm{sc}^{+}-$sections and the associated set of positive rational numbers $\left(\sigma_{i}\right)_{i \in I}$ so that $\Lambda(e)=\sum_{\left\{i \mid s_{i}(P e)=e\right\}} \sigma_{i}$ and we define

$$
N^{*}(\Lambda)(y)=\max _{i \in I} N^{*}\left(s_{i}(y)\right), \quad y \in U_{x} .
$$

This definition is independent of the choice of the local section structure. Indeed, if $\left(t_{j}\right)_{j \in J}$ is another local sections structure on $U_{x}$ defining the multisection $\Lambda$ and $y \in U_{x}$, then for every $i \in I$ there exists an index $j \in J$ such that $s_{i}(y)=t_{j}(y)$. Conversely, for every $j \in J$ there is an index $i \in I$ such that $t_{j}(y)=s_{i}(y)$. Hence $\max _{i \in I} N^{*}\left(s_{i}(y)\right)=\max _{j \in J} N^{*}\left(t_{i}(y)\right)$ as claimed. Since the morphisms extend to local sc-diffeomorphisms, it follows that $N^{*}(\Lambda)$ is invariant under morphisms, that is, $N^{*}(\Lambda)(x)=N^{*}(\Lambda)\left(x^{\prime}\right)$ if there exists a morphism $\varphi: x \rightarrow x^{\prime}$. Consequently, we define the auxiliary norm of the $\mathrm{sc}^{+}$-multisection $\lambda$ by

$$
N(\lambda)(z)=N^{*}(\Lambda)(x) \text { for } z=|x| .
$$


Lemma 4.16 Assume that $f: Z \rightarrow W$ is a proper Fredholm section of the strong polyfold bundle $p: W \rightarrow Z$ and that the pair $(U, N)$ controls compactness. Then given an $\mathrm{sc}^{+}$-multisection $\lambda$ satisfying $N(\lambda) \leq 1$, the solution set

$$
S(f, \lambda)=\{z \in Z \mid \lambda(f(z))>0\}
$$

is compact.

Proof It suffices to prove the result in local models. Hence we assume that $P: E \rightarrow X$ is a local model for $p$ and that $F: X \rightarrow E$ is a proper Fredholm section representing $f$ and we assume that $\Lambda: E \rightarrow \mathbb{Q}^{+}$is an $\operatorname{sc}^{+}$-multisection representing $\lambda$. Then $N^{*}(\Lambda)=N(\lambda) \leq 1$. Set $U^{*}=\pi^{-1}(U)$. The result will follow by showing that the solution set

$$
S(F, \Lambda)=\{x \in X \mid \Lambda(F(x))>0\}
$$

is compact. To see this we take a sequence $\left(x_{n}\right) \subset S(F, \Lambda)$. Then $\Lambda\left(F\left(x_{n}\right)\right)>0$. Hence given the point $x_{n}$, there exists a neighborhood $U_{x_{n}}$ and a local section structure $\left(s_{i}\right)_{i \in I}$ together with associated weights $\left(\sigma_{i}\right)_{i \in I}$ so that $\Lambda\left(F\left(x_{n}\right)\right)=\sum \sigma_{i}$ where the sum is taken over the indices $i$ for which $F\left(x_{n}\right)=s_{i}\left(x_{n}\right)$. From $\Lambda\left(F\left(x_{n}\right)\right)>0$ one concludes $F\left(x_{n}\right)=s_{i}\left(x_{n}\right)$ for some $i$, and from $N^{*}(\Lambda) \leq 1$ one concludes $N^{*}\left(F\left(x_{n}\right)\right) \leq 1$ for all $n$. By assumption, the pair $(U, N)$ controls compactness so that by Lemma 4.4 there is a sequence of points $\left(y_{n}\right) \in U^{*}$ and a sequence of morphisms $\varphi_{n}: x_{n} \rightarrow y_{n}$ such that $\left(y_{n}\right)$ contains a converging subsequence. We assume without loss of generality that $y_{n} \rightarrow y$. In an open neighborhood $U_{y}$ of $y$ there is a local section structure $\left(t_{j}\right)_{j \in J}$ with associated weights $\left(\tau_{j}\right)_{j \in J}$ so that $\Lambda(F(z))=\sum_{\left\{j \mid t_{j}(z)=F(z)\right\}} \tau_{j}$ for $z \in U_{y}$. For large $n, x_{n} \in U_{y}$ and since the index set $J$ is finite, $F\left(x_{n}\right)=t_{j_{0}}\left(x_{n}\right)$ for some fixed index $j_{0} \in J$ and some subsequence, denoted again by $\left(x_{n}\right)$. Hence $F(y)=t_{j_{0}}(y)$ showing that $\Lambda(F(y))>0$. Moreover, $y \in U^{*}$ since the support of $\Lambda$ is contained in $U^{*}$. This completes the proof.

We are ready to prove the perturbation result in the case of no boundary, $\partial Z=\varnothing$. In order to have sc-smooth functions and sc-smooth sections with supports in preassigned open sets available (see the Appendix, Section 5.2), we shall assume in the following that the sc-structures of the ep-groupoids $X$ used as models of the polyfold $Z$ are based on sc-separable Hilbert spaces. However, we would like to point out that what is really required is that the ep-groupoids are modeled on sc-scales $\left(E_{j}\right)_{j \geq 0}$ in which only the space $E_{0}$ is required to be a separable Hilbert space.

Theorem 4.17 Let $f$ be a proper (oriented) Fredholm section of the strong polyfold bundle $p: W \rightarrow Z$ having empty boundary and assume that the polyfold structure $Z$ is based on separable Hilbert spaces. Fix an auxiliary norm $N$ for $p$ and an open 
neighborhood $U$ of the solution set $S(f)=\{z \in Z \mid f(z)=0\}$ so that the pair $(U, N)$ controls compactness. Assume that $\varepsilon \in\left(0, \frac{1}{2}\right)$. Then for every sc ${ }^{+}$-multisection $\lambda$ supported in $U$ and satisfying $N(\lambda)<\frac{1}{2}$, there exists an $\mathrm{sc}^{+}-$multisection $\tau: W \rightarrow \mathbb{Q}^{+}$ supported in $U$ and satisfying $N(\tau)<\varepsilon$, so that the pair $(f, \lambda \oplus \tau)$ is a transversal pair. In particular, the associated solution set

$$
S(f, \lambda \oplus \tau)=\{z \in Z \mid(\lambda \oplus \tau)(f(z))>0\}
$$

is an (oriented) compact branched suborbifold of $Z$ without boundary.

Proof Take an $\mathrm{sc}^{+}$-multisection $\lambda: W \rightarrow \mathbb{Q}^{+}$satisfying $N(\lambda)<\frac{1}{2}$. In view of Lemma 4.16, the solution set

$$
S(f, \lambda)=\{z \in Z \mid \lambda(f(z))>0\}
$$

is compact subset of the polyfold $Z$.

We choose a strong polyfold bundle $P: E \rightarrow X$ over the ep-groupoid $X$ as a model for $p: W \rightarrow Z$ and let the proper Fredholm section $F: X \rightarrow E$ of the bundle $P$ represent the proper Fredholm section $f$ of $p$. We may assume without loss of generality that

$$
Z=|X| \quad \text { and } \quad W=|E| .
$$

Assume that the $\mathrm{sc}^{+}$-multisection $\Lambda: E \rightarrow \mathbb{Q}^{+}$of $P$ represents the $\mathrm{sc}^{+}$-multisection $\lambda$ of $p$. If $\pi: X \rightarrow|X|$ denotes the quotient map onto the orbit space, the set $U^{*}=\pi^{-1}(U)$ is an open neighborhood of the compact solution set

$$
S(F, \Lambda)=\{x \in X \mid \Lambda(F(x))>0\},
$$

and since $N(\lambda)=N^{*}(\Lambda)$, we have $N^{*}(\Lambda)<\frac{1}{2}$.

We fix a solution $x \in S(F, \Lambda)$ and take an open neighborhood $U_{x} \subset X$ of $x$ on which the isotropy group $G_{x}$ acts by its natural representation $\varphi_{g} \in \operatorname{Diff}_{\mathrm{sc}}\left(U_{x}\right)$. The local system of sections of $U_{x}$ we shall denote by $\left(a_{i}\right)_{i \in I}$ and the associated weights by $\left(\sigma_{i}\right)_{i \in I}$. Then, if $y \in U_{x}$,

$$
\Lambda(F(y))=\sum_{\left\{i \in I \mid F(y)=a_{i}(y)\right\}} \sigma_{i} .
$$

Moreover, $\Lambda(F(y))=0$ if there is no index $i \in I$ satisfying $F(y)=a_{i}(y)$. By assumption on the solution, $\Lambda(F(x))>0$, so that there is a subset $J \subset I$ such that

$$
F(x)-a_{i}(x)=0 \quad \text { for all } i \in J .
$$


Recall that for $g \in G_{x}$ the map $\mu(g, \cdot): E_{x} \rightarrow E_{x}$ is a linear sc-isomorphism. Therefore, we can choose smooth linearly independent vectors $e^{1}, \ldots, e^{m} \in E_{x}$ such that for every $g \in G_{x}$ the vectors

$$
\mu\left(g, e^{1}\right), \ldots, \mu\left(g, e^{m}\right)
$$

in $E_{x}$ span the cokernels of the linearizations

$$
\left(F-a_{i}\right)^{\prime}(x)
$$

for every $i \in I^{\prime}$. If $g=\mathrm{id} \in G_{x}$ is the identity element of the isotropy group $G_{x}$, then $\mu\left(\mathrm{id}, e^{j}\right)=e^{j}$ for $1 \leq j \leq m$.

By means of Lemma 3.36, we find $\mathrm{sc}^{+}$-sections $s^{1}, \ldots, s^{m}: U_{x} \rightarrow E \mid U_{x}$ having their supports in $U_{x}$ and satisfying $s^{j}(x)=e^{j}$ for $1 \leq j \leq m$.

For every $g \in G_{x}$, we next define the $\mathrm{sc}^{+}{ }_{-}$sections $s_{g}^{j}: U_{x} \rightarrow E \mid U_{x}$ by

$$
s_{g}^{j}\left(\varphi_{g}(y)\right):=\mu\left(\Gamma(g, y), s^{j}(y)\right),
$$

where $y \in U_{x}$ and $1 \leq j \leq m$. Introducing the sum

$$
s_{g}^{t}(y):=\sum_{j=1}^{m} t_{j} s_{g}^{j}(y),
$$

where $y \in U_{x}$ and $t=\left(t_{1}, \ldots, t_{n}\right) \in \mathbb{R}^{n}$, we have defined the $\sharp G_{x}$-many parametrized $\mathrm{sc}^{+}$-sections $s_{g}^{t}: U_{x} \rightarrow E \mid U_{x}$ having their supports in $U_{x}$. If id $\in G_{x}$ is the identity element, then the morphism $\Gamma$ (id, $y$ ) is equal to $1_{y}: y \rightarrow y$ and using that $\mu\left(1_{y}, e_{y}\right)=$ $e_{y}$ for all $e_{y} \in E_{y}$, the $\mathrm{sc}^{+}$-sections $s_{g}^{t}$ satisfy

with

$$
\begin{aligned}
s_{g}^{t}\left(\varphi_{g}(y)\right) & =\mu\left(\Gamma(g, y), s_{\mathrm{id}}^{t}(y)\right) \\
s_{\mathrm{id}}^{t}(y) & =\sum_{j=1}^{m} t_{j} s^{j}(y) .
\end{aligned}
$$

The map

$$
\Lambda_{U_{x}}^{t}: E \mid U_{x} \rightarrow \mathbb{Q}^{+}
$$

is defined by

$$
\Lambda_{U_{x}}^{t}(e)=\frac{1}{\sharp G_{x}} \cdot \sharp\left\{g \in G_{x} \mid s_{g}^{t}(P(e))=e\right\} .
$$

Proceeding as in Proposition 3.38, one extends the local multisection $\Lambda_{U_{x}}^{t}$ from $E \mid U_{x}$ to the parametrized $\mathrm{sc}^{+}$-multisection

$$
\Lambda_{x}^{t}: E \rightarrow \mathbb{Q}^{+} .
$$


Continuing with the proof of Theorem 4.17, we introduce for $i \in J$ and $g \in G_{x}$ the perturbed sections $F_{g}^{i}: \mathbb{R}^{m} \oplus U_{x} \rightarrow E \mid U_{x}$ by

$$
F_{g}^{i}(t, y)=F(y)-a_{i}(y)-\sum_{j=1}^{m} t_{j} s_{g}^{j}(y)
$$

where $t=\left(t_{1}, \ldots, t_{m}\right) \in \mathbb{R}^{m}$. In the special case $g=\mathrm{id} \in G_{x}$ one has

$$
F_{\mathrm{id}}^{i}(t, y)=F(y)-a_{i}(y)-\sum_{j=1}^{m} t_{j} s^{j}(y) .
$$

In view of Theorem 3.9 in [22], the perturbed sections $F_{g}^{i}$ are proper Fredholm sections of the bundle $E^{1} \rightarrow \mathbb{R}^{m} \oplus X^{1}$ over the set $\mathbb{R}^{m} \oplus\left(U_{x}\right)^{1}$.

Lemma 4.18 For every $i \in J$ and $g \in G_{x}$ the linearization

$$
D F_{g}^{i}(0, x): T_{0} \mathbb{R}^{m} \oplus T_{x} X \rightarrow E_{x}
$$

of the map $(t, y) \mapsto F_{g}^{i}(t, y)$ at the special point $(0, x)$ at which $F(x)-a_{i}(x)=0$, is surjective.

Proof The linearization of the map $(t, y) \mapsto F_{g}^{i}(t, y)$ is equal to

$$
D F_{g}^{i}(t, y) \cdot(\delta t, \delta y)=\left(F^{\prime}(y)-a_{i}^{\prime}(y)\right) \cdot \delta y-\sum_{j=1}^{m} \delta t_{j} \cdot s_{g}^{j}(y)-\sum_{j=1}^{m} t_{j} \cdot D_{y} s_{g}^{j}(y) \cdot \delta y,
$$

where $\delta t=\left(\delta t_{1}, \ldots, \delta t_{m}\right) \in \mathbb{R}^{m}$ and $\delta y \in T_{x} X$. At the point $y=x$ we have, using $\varphi_{g}(x)=x$, that $s_{g}^{j}(x)=\mu\left(\Gamma(g, x), s^{j}(x)\right)=\mu\left(g, s^{j}(x)\right)=\mu\left(g, e^{j}\right)$. Therefore the linearization at $(t, y)=(0, x)$ is represented by the linear map

$$
D F_{g}^{i}(0, x) \cdot(\delta t, \delta y)=\left(F^{\prime}(x)-a_{i}^{\prime}(x)\right) \cdot \delta y-\sum_{j=1}^{m} \delta t_{j} \cdot \mu\left(g, e^{j}\right),
$$

which, in view of the definition of $\mu\left(g, e^{j}\right)$ for $1 \leq j \leq m$, is obviously surjective as claimed in the lemma.

Since by Lemma 4.18 the linearizations $D F_{g}^{i}(0, x)$ at the point $(0, x)$ are surjective, it follows, by the arguments in Section 4.2 in [22], that the linearizations $D F_{g}^{i}(t, y)$ at the points solving $F_{g}^{i}(t, y)=0$ are also surjective if $|t|$ is small and $y$ belongs to a possibly smaller invariant neighborhood $V_{x} \subset U_{x}$ of the distinguished point $x$.

By assumption, the solution set $S(f, \lambda)$ of the proper Fredholm section $f$ of $p$ is compact. Consequently, there exist finitely many solutions $x_{1}, \ldots, x_{m}$ belonging 
to the solution set $S=S(F, \Lambda)$ so that for the corresponding open neighborhoods $V_{x_{1}}, \ldots, V_{x_{m}}$ in $X$, the subsets $\left|V_{x_{1}}\right|, \ldots,\left|V_{x_{m}}\right|$ of the orbit space $|X|$ cover $f^{-1}(0)$. Then we define the parametrized $\mathrm{sc}^{+}$-multisection $\Lambda_{t}$ as the sum

$$
\Lambda_{t}=\Lambda_{x_{1}}^{t_{1}} \oplus \cdots \oplus \Lambda_{x_{m}}^{t_{m}}
$$

where $t=\left(t_{1}, \ldots, t_{m}\right) \in \mathbb{R}^{n_{1}} \times \cdots \mathbb{R}^{n_{m}}=\mathbb{R}^{N}$ with $N=n_{1}+\cdots+n_{m}$. By construction, at every point $(t, x)=(0, x) \in\{0\} \times S$, for the new parametrized local branching structure at $x \in X$, we have surjectivity of the linearizations of every local problem, so that the set $M=\left\{(t, x) \in \mathbb{R}^{N} \times X \mid\left(\Lambda \oplus \Lambda_{t}\right)(F(x))>0\right\}$ and $|t|$ small $\}$ is locally a collection of finitely many finite dimensional submanifolds of $X$. The projection maps $M \rightarrow \mathbb{R}^{N}$ given by $(t, x) \mapsto t$ can be viewed as finitely many maps defined on smooth submanifolds so that it makes sense to talk about regular values. We choose by means of Sard's theorem a small regular value $t^{*} \in \mathbb{R}^{N}$. Then the pairs $\left(F, \Lambda_{t^{*}}\right)$ and $\left(f, \lambda_{t^{*}}\right)$ are transversal and the map $\Theta: X \rightarrow \mathbb{Q}^{+}$defined by $\Theta(x)=\left(\Lambda \oplus \Lambda_{t^{*}}\right)(F(x))$ is a branched ep-subgroupoid of the ep-groupoid $X$. This completes the proof of Theorem 4.17.

We point out that, in view of Proposition 4.9, the perturbation $\Lambda^{t}$ is independent of local section structures of the unperturbed $\mathrm{sc}^{+}$-multisection $\Lambda$.

Next we consider the case in which the polyfold $Z$ possesses a boundary $\partial Z$.

Theorem 4.19 Let $f$ be a proper (oriented) Fredholm section of the strong polyfold bundle $p: W \rightarrow Z$ with boundary with corners and assume that the polyfold structure for $Z$ is built on separable Hilbert spaces. Fix an auxiliary norm $N$ for $p$ and an open neighborhood $U$ of the solution set $S(f)=\{z \in Z \mid f(z)=0\}$ so that the pair $(U, N)$ controls compactness. Then for every $\mathrm{sc}^{+}-$multisection $\lambda$ supported in $U$ and satisfying $N(\lambda)<\frac{1}{2}$ and every $\varepsilon \in\left(0, \frac{1}{2}\right)$, there exists an $\mathrm{sc}^{+}$-multisection $\tau$ supported in $U$ and satisfying $N(\tau)<\varepsilon$, so that $(f, \lambda \oplus \tau)$ is in general position. In particular, the solution set

$$
S(f, \lambda \oplus \tau)=\{z \in Z \mid(\lambda \oplus \tau)(f(z))>0\}
$$

is an (oriented) compact branched suborbifold of the polyfold $Z$ with boundary with corners.

The proof is a variation of the corresponding proof of Theorem 5.22 in [22] for Fredholm sections on M-polyfold bundles. Before we start proving the theorem we recall some notation. If $x$ is a point of the M-polyfold $X$, we denote by $d=d(x)$ the degeneracy index of $x$ defined in Section 3.1. By $\mathcal{F}^{1}, \ldots, \mathcal{F}^{d}$ we abbreviate the local faces of $X$ at the point $x$. Every local face $\mathcal{F}^{j}$ has the tangent space $T_{x} \mathcal{F}^{j}$ at the point 
$x \in X$. The subset $T_{x_{j}}^{\partial} X \subset T_{x} X$ is the intersection $T_{x}^{\partial} X=\bigcap_{1 \leq j \leq d} T_{x} \mathcal{F}^{j}$. In the case that $x$ is an interior point, the degeneration index $d(x)$ is equal to 0 and we set $T_{x}^{\partial} X=T_{x} X$.

Proof Proceeding as in the proof of Theorem 4.17, we let $\lambda: W \rightarrow \mathbb{Q}^{+}$be an sc $\mathrm{sc}^{+}$ multisection satisfying $N(\lambda)<\frac{1}{2}$. Since $(N, U)$ controls compactness, the solution set $S(f, \lambda)$ is a compact subset of $|X|$ in view of Lemma 4.16. Set $U^{*}=\pi^{-1}(U)$. Let $\Lambda: E \rightarrow \mathbb{Q}^{+}$be the $\mathrm{sc}^{+}$-multisection representing the $\mathrm{sc}^{+}$-multisection $\lambda: W \rightarrow \mathbb{Q}^{+}$ in the strong polyfold bundle $P: E \rightarrow X$. We assume that the proper Fredholm section $F: X \rightarrow E$ of $P$ represents the proper Fredholm section $f: Z \rightarrow W$ of $p$. Again we may assume that $Z=|X|$ and $W=|E|$.

Fix a solution $x$ belonging to the compact solution set $S(F, \Lambda)$ and take an open neighborhood $U_{x} \subset X$ of $x$ which is invariant under the action of the isotropy group $G_{x}$. On the neighborhood $U_{x}$ we have a local system of sections $\left(a_{i}\right)$ for $i \in I$ with associated weights $\left(\sigma_{i}\right)_{i \in I}$, so that

$$
\Lambda(F(x))=\sum_{\left\{i \in I \mid F(x)=a_{i}(x)\right\}} \sigma_{i} .
$$

A subset $J$ of the index set $I$ such that $F(x)-a_{i}(x)=0$ for all $i \in J$ is nonempty because $\Lambda(F(x))>0$.

Next we construct the parametrized $\mathrm{sc}^{+}$-perturbation of the section $F-a_{i}$ for all $i \in J$. As in the proof of Theorem 4.17, choose smooth linearly independent vectors $e^{1}, \ldots, e^{m} \in E$ with the property that for all $g \in G_{X}$ the vectors $\mu\left(g, e^{1}\right), \ldots, \mu\left(g, e^{m}\right)$ span all the cokernels of the linearizations $F^{\prime}(x)-a_{i}^{\prime}(x): T_{x} X \rightarrow E_{x}$ for every $i \in J$. By Lemma 3.36, there are $\mathrm{sc}^{+}{ }_{-}$sections $s^{1}, \ldots, s^{m}: U_{x} \rightarrow E \mid U_{x}$ having their supports in $U_{x}$ and satisfying $s^{j}(x)=e^{j}$ for $j=1, \ldots, m$. To achieve transversality of the kernels of the linearized perturbed Fredholm sections we choose additional sc ${ }^{+}-$ sections $s^{m+1}, \ldots, s^{N}: U_{x} \rightarrow E \mid U_{x}$ having their supports in $U_{x}$. This is done as follows. First observe that the kernel of the linear map

$$
(\lambda, h) \mapsto\left(F-a_{i}\right)^{\prime}(x) \cdot h+\sum_{j=1}^{N} \lambda_{j} \cdot s^{j}(x)
$$

consists of $\{0\} \oplus \operatorname{ker}\left(F-a_{i}\right)^{\prime}(x)$ together with the vectors $(\lambda, h)$ which are the solutions of the equation $\left(F-a_{i}\right)^{\prime}(x)=-\sum_{j=1}^{N} \lambda_{j} \cdot s^{j}(x)$. Since the space $T_{x}^{\partial} X$ is of finite codimension in the tangent space $T_{X} X$, we find finitely many smooth linearly independent vectors $h^{m+1}, \ldots, h^{n}$ so that

$$
\operatorname{span}\left\{h^{m+1}, \ldots, h^{n}\right\} \oplus T_{x}^{\partial} X=T_{x} X .
$$


With this choice of the vectors $h^{m+1}, \ldots, h^{n}$, we define the smooth vectors $e^{i, l, g} \in E_{x}$ by

$$
e^{i, l, g}:=-\mu\left(g,\left(F-a_{i}\right)^{\prime}(x) h^{l}\right)
$$

for all $i \in J, m+1 \leq l \leq n$, and $g \in G_{x}$. We extend, using Lemma 3.36, these vectors to $\mathrm{sc}^{+}$-sections having their supports in $U_{x}$ and label them by $s^{m+1}, \ldots, s^{N}$.

Next we introduce the perturbed sc-smooth section $F_{i}: \mathbb{R}^{N} \oplus U_{x} \rightarrow E$ by

$$
F_{i}(t, y):=F(y)-a_{i}(y)+\sum_{j=1}^{N} t_{j} \cdot s^{j}(y),
$$

where $y \in U_{x}$ and $t=\left(t_{1}, \ldots, t_{N}\right) \in \mathbb{R}^{N}$. This is a proper Fredholm section having the following additional properties. Its linearization $F_{i}^{\prime}(0, x)$ at the point $(0, x)$ is surjective and its kernel is transversal to $\mathbb{R}^{N} \oplus T_{x}^{\partial} X$ in $\mathbb{R}^{N} \oplus T_{x} X$ for every $i \in J$. Moreover, for every subset $\tau$ of the set $\{1, \ldots, d(x)\}$, the linearization $F_{i}^{\prime}(0, x)$ restricted to the tangent space $T_{(0, x)}\left(\mathbb{R}^{N} \oplus \bigcap_{j \in \tau} \mathcal{F}^{j}\right)$ is surjective and the kernel of this restriction is transversal to the subspace $T_{(0, x)}^{\partial}\left(\mathbb{R}^{N} \oplus \bigcap_{j \in \tau} \mathcal{F}^{j}\right)$.

For $g \in G_{x}$ and $1 \leq j \leq N$, we define the $\mathrm{sc}^{+}$-section $s_{g}^{j}: U_{x} \rightarrow E \mid U_{x}$ by

$$
s_{g}^{j}\left(\varphi_{g}(y)\right):=\mu\left(\Gamma(g, y), s^{j}(y)\right)
$$

and the $\mathrm{sc}^{+}-$section $s_{g}^{t}$ by

$$
s_{g}^{t}(y):=\sum_{j=1}^{N} t_{j} \cdot s_{g}^{j}(y)
$$

for $y \in U_{x}$ and $t=\left(t_{1}, \ldots, t_{N}\right) \in \mathbb{R}^{N}$. Hence we have the $\sharp G_{x}$-many parametrized $\mathrm{sc}^{+}$-sections $U_{x} \rightarrow E \mid U_{x}$ having supports in $U_{x}$, which define the map $\Lambda_{U_{x}}^{t}: E \mid U_{x} \rightarrow$ $\mathbb{Q}^{+}$by

$$
\Lambda_{U_{x}}^{t}(e)=\frac{1}{\sharp G_{x}} \cdot \sharp\left\{g \in G_{x} \mid s_{g}^{t}(P(e))=e\right\} .
$$

As in Proposition 3.38 we extend the local sc ${ }^{+}$-multisection $\Lambda_{U_{x}}^{t}$ from $E \mid U_{x}$ to the parametrized $\mathrm{sc}^{+}-$multisection

$$
\Lambda_{x}^{t}: E \rightarrow \mathbb{Q}^{+} .
$$

If $i \in J$ and $g \in G_{x}$, we introduce the perturbed section $F_{g}^{i}: \mathbb{R}^{N} \times U_{x} \rightarrow E$ by

$$
F_{g}^{i}(t, y)=F(y)-a_{i}(y)+s_{g}^{t}(y) .
$$

It is a proper Fredholm section which as we show next has the same properties as the section $F_{i}$. 
Lemma 4.20 The linearization $D F_{g}^{i}(0, x): \mathbb{R}^{N} \oplus T_{x} X \rightarrow E_{x}$ is surjective and its kernel is transversal to $\mathbb{R}^{N} \oplus T_{x}^{\partial} X$ in $\mathbb{R}^{N} \oplus T_{x} X$. Moreover, for every subset $\tau$ of the set $\{1, \ldots, d(x)\}$, the restriction of $D F_{g}^{i}(0, x)$ to the tangent space $T_{(0, x)}\left(\mathbb{R}^{N} \oplus \bigcap_{j \in \tau} \mathcal{F}^{j}\right)$ is surjective and the kernel of this restriction is transversal to the subspace $T_{(0, x)}^{\partial}\left(\mathbb{R}^{N} \oplus \bigcap_{j \in \tau} \mathcal{F}^{j}\right)$.

Proof Fix $i \in J$ and $g \in G_{x}$. In view of the fact that $\operatorname{span}\left\{h^{m+1}, \ldots, h^{n}\right\} \oplus T_{x}^{\partial} X=$ $T_{X} X$, it suffices to show that for every vector $h^{l}$, there is a point $\lambda^{l} \in \mathbb{R}^{N}$ such that $\left(\lambda^{l}, h^{l}\right) \in \mathbb{R}^{N} \oplus T_{x} X$ belongs to the kernel of the linearization $\left(F_{g}^{i}\right)^{\prime}(0, x)$. Recall that vectors $e^{i, l, h}$ for $i \in J, m+1 \leq l \leq n$ and $h \in G_{x}$ are denoted as $s^{j}(x)$ with $m+1 \leq j \leq N$. Hence given $m+1 \leq l \leq n$ there is an index $m+1 \leq j \leq N$ such that $e^{i, l, g^{-1}}=s^{j}(x)$. By definition, $e^{i, l, g^{-1}}=\mu\left(g^{-1},\left(F-a_{i}\right)^{\prime}(x) h^{l}\right)$ so that

$$
\begin{aligned}
-s_{g}^{j}(x) & =-\mu\left(g, s^{j}(x)\right)=\mu\left(g, \mu\left(g^{-1},\left(F-a_{i}\right)^{\prime}(x) h^{l}\right)\right) \\
& =\mu\left(g^{-1} \circ g,\left(F-a_{i}\right)^{\prime}(x) h^{l}\right)=\left(F-a_{i}\right)^{\prime}(x) h^{l} .
\end{aligned}
$$

Hence if $\lambda^{l} \in \mathbb{R}^{N}$ is defined by $\lambda_{k}^{l}=1$ if $k=j$ and $\lambda_{k}^{l}=0$ for $k \neq j$, the pair $\left(\lambda^{l}, h^{l}\right)$ belongs to the kernel of the linearizations $\left(F_{g}^{i}\right)^{\prime}(0, x)$ as claimed. The remaining part of the lemma is proved in a similar way.

Lemma 4.21 There exist a $G_{x}$-invariant open neighborhood $V_{x} \subset U_{x}$ and a parametrized $\mathrm{sc}^{+}$-multisection $\Lambda_{x}^{t}: E \rightarrow \mathbb{Q}^{+}$which is supported in $U_{x}$ and linear in $t$ such that for $\varepsilon>0$ sufficiently small the solution set

$$
S_{x, \varepsilon}=\left\{(t, y) \in \mathbb{R}^{N} \times X^{1}\left|\left(\Lambda \oplus \Lambda_{x}^{t}\right)(F(y))>0,\right| t \mid<\varepsilon, \text { and } y \in V_{x}\right\}
$$

has the following properties. For every $i \in I$ and $g \in G_{x}$ and $(t, y) \in S_{x, \varepsilon}$ solving the equation

$$
F_{g}^{i}(t, y)=F(y)-a_{i}(y)+s_{g}^{t}(y)=0
$$

the following holds true:

(1) The linearization $D F_{g}^{i}(t, y)$ is surjective.

(2) The kernel of $D F_{g}^{i}(t, y)$ is transversal to the subspace $T_{(t, y)}^{\partial}\left(\mathbb{R}^{N} \times X\right)$ of the tangent space $T_{(t, y)}\left(\mathbb{R}^{N} \times X\right)$.

(3) For every subset $\sigma$ of $\{1, \ldots d(y)\}$, the linearization of $D F_{g}^{i}(t, y)$ restricted to the tangent space $T_{(t, y)}\left(\mathbb{R}^{N} \times \bigcap_{j \in \sigma} \mathcal{F}^{j}\right)$ is surjective, and the kernels of these restrictions are transversal to the subspace $T_{(t, y)}^{\partial}\left(\mathbb{R}^{N} \times \bigcap_{j \in \sigma} \mathcal{F}^{j}\right)$ in the tangent space $T_{(t, y)}\left(\mathbb{R}^{N} \oplus \bigcap_{j \in \sigma} \mathcal{F}^{j}\right)$. 
Proof We already know from Lemma 4.20 that the conclusions of the lemma hold true at the special point $(t, y)=(0, x)$. Working in local coordinates as in the proof of Lemma 5.23 in [22], we find for every $i \in J$ and $g \in G_{x}$ a positive number $\varepsilon_{i, g}$ and an open neighborhood $V_{i, g} \subset U_{x}$ of $x$ such that the conclusions (1)-(3) hold for $(t, y) \in \mathbb{R}^{N} \oplus V_{i, g}$ if $|t|<\varepsilon_{i, g}$. Then the lemma follows by taking for the set $V_{x}$ a $G_{x}$-invariant open neighborhood of $x$ which is contained in the intersection of the sets $V_{j, g}$ and choosing for $\varepsilon$ a positive number smaller than the numbers $\varepsilon_{i, g}$.

Finally we can finish the proof of Theorem 4.19.

By assumption, the solution set $S(f, \lambda)$ of the proper Fredholm section $f$ of the bundle $p$ is a compact subset of the orbit space $|X|$. Consequently, there exist finitely many solutions $x_{1}, \ldots, x_{m}$ belonging to $S(F, \Lambda)$ so that for the corresponding open neighborhoods $V_{x_{1}}, \ldots, V_{x_{m}}$ in $X$, the subsets $\left|V_{x_{1}}\right|, \ldots,\left|V_{x_{m}}\right|$ of the orbit space $|X|$ cover $S(f, \lambda)$. Then we define the parametrized sc ${ }^{+}$-multisection $\Lambda_{t}$ as the sum

$$
\Lambda_{t}=\Lambda_{x_{1}}^{t_{1}} \oplus \cdots \oplus \Lambda_{x_{m}}^{t_{m}}
$$

where $t=\left(t_{1}, \ldots, t_{m}\right) \in \mathbb{R}^{N_{1}} \times \cdots \mathbb{R}^{N_{m}}=\mathbb{R}^{N}$ with $N=N_{1}+\cdots+N_{m}$.

Using Lemma 4.21 one concludes that the solution set

$$
S_{\varepsilon}=\left\{(t, y) \in \mathbb{R}^{N} \times X^{1} \mid\left(\Lambda \oplus \Lambda_{t}\right)(F(y))>0 \text { and }|t|<\varepsilon\right\}
$$

consists of a finite collection of finite dimensional manifolds with boundary with corners. Now one studies the projection map $S_{\varepsilon} \rightarrow \mathbb{R}^{l}$ defined by $(t, x) \mapsto t$ and finds a small common regular value $t^{*}$ for various restrictions of the map to intersections of local faces. For this parameter value $t^{*}$, the pair $\left(F, \Lambda \oplus \Lambda_{t^{*}}\right)$ is in general position and the associated solution space has an orbit space which is a compact branched suborbifold of the polyfold $Z=|X|$ in general position to the boundary. In particular, the associated solution set $S\left(f, \lambda \oplus \vartheta_{t^{*}}\right)=\left\{z \in Z \mid\left(\lambda \oplus \vartheta_{t^{*}}\right)(f(z))>0\right\}$ is a compact branched orbifold with boundary with corners. This finishes the proof of Theorem 4.19.

The following result is proved along the lines of the previous result. In contrast to Theorem 4.19, we impose conditions on the Fredholm sections at those solutions which are located at the boundary $\partial X$, while the perturbation has its support away from the boundary.

Let $f$ be a proper (oriented) Fredholm section of the strong polyfold bundle $p$ : $W \rightarrow Z$ with boundary with corners and assume that the polyfold structure for $Z$ is built on separable Hilbert spaces. Fix an auxiliary norm $N$ for $p$ and an open neighborhood $U$ of the solution set $S(f)=\{z \in Z \mid f(z)=0\}$ so that the pair $(U, N)$ controls 
compactness. Then for every $\mathrm{sc}^{+}$-multisection $\lambda$ supported in $U$ and satisfying $N(\lambda)<\frac{1}{2}$ and every $\varepsilon \in\left(0, \frac{1}{2}\right)$, there exists an $\mathrm{sc}^{+}$-multisection $\tau$ supported in $U$ and satisfying $N(\tau)<\varepsilon$, so that so that $(f, \lambda \oplus \tau)$ is in general position. In particular, the solution set

$$
S(f, \lambda \oplus \tau)=\{z \in Z \mid(\lambda \oplus \tau)(f(z))>0\}
$$

is an (oriented) compact branched suborbifold of the polyfold $Z$ with boundary with corners.

Theorem 4.22 Let $f$ be a proper (oriented) Fredholm section of the strong polyfold bundle $p: W \rightarrow Z$ with boundary with corners and assume that the polyfold structure for $Z$ is built on separable Hilbert spaces. Fix an auxiliary norm $N$ for $p$ and an open neighborhood $U$ of the solution set $S(f)=\{z \in Z \mid f(z)=0\}$ so that the pair $(U, N)$ controls compactness. Assume that $\lambda$ is an $\mathrm{sc}^{+}-$multisection supported in $U$, satisfying $N(\lambda)<\frac{1}{2}$, and such that the pair $(f, \lambda)$ is in good position. Then, given $\varepsilon \in\left(0, \frac{1}{2}\right)$, there exists an $\mathrm{sc}^{+}$-multisection $\tau$ supported in $U$ and satisfying $N(\tau)<\varepsilon$, so that $\tau$ is trivial at the boundary and the pair $(f, \lambda \oplus \tau)$ is in good position. In particular, the solution set $S(f, \lambda \oplus \tau)$ is an (oriented) compact branched suborbifold of $Z$ with boundary with corners.

Proof The proof is straightforward. By assumption, the pair $(f, \lambda)$ is already transversal and in good position to the boundary so that the solution space of $(f, \lambda)$ near $\partial Z$ is already a branched orbifold. Then we can perturb the $\mathrm{sc}^{+}-$multisection $\lambda$ by an arbitrarily small $\mathrm{sc}^{+}$-multisection $\tau$ by the same argument as in the proof of Theorem 4.17, where, in addition, $\tau$ is trivial near $\partial Z$, so that the pair $(f, \lambda \oplus \tau)$ is transversal and, by construction, still in good position at solutions $x \in \partial Z$. The last statement is proved along the lines of Theorem 4.14 using [22, Theorem 5.16].

Theorem 4.22 and variants thereof are important in the "coherent perturbation theory" used in Symplectic Field Theory where one deals simultaneously with infinitely many Fredholm problems and where the boundaries are explained as products (or fibered products) of Fredholm problems. In this case one has an algorithm how the data are being perturbed which defines inductively perturbations on the boundary so that the problem at the boundary is already in good position. Then one extends the perturbation by keeping compactness and transversality.

\subsection{Invariants}

The results above allow to define invariants. The first result is an abstract version of the argument used in order to define the Gromov-Witten invariants in [15]. 
Theorem 4.23 Assume that $f$ is a proper oriented Fredholm section of the strong polyfold bundle $p: W \rightarrow Z$ and $\partial Z=\varnothing$.

Then there exists a well-defined map

$$
\Phi_{f}: H_{\mathrm{dR}}^{*}(Z) \rightarrow \mathbb{R}
$$

defined on the deRham cohomology group $H_{\mathrm{dR}}^{*}(Z)$ so that the following holds. If $(N, U)$ is a pair controlling compactness, where $N$ is an auxiliary norm and $U$ a corresponding open neighborhood of the solution set $S(f)=\{z \in Z \mid f(z)=0\}$, then for any sc ${ }^{+}-$multisection $\lambda$ with support in $U$ and satisfying $N(\lambda)<1$ and such that the pair $(f, \lambda)$ is transversal, the following representation of $\Phi_{f}$ holds:

$$
\Phi_{f}([\omega]):=\int_{\left(S(f, \lambda), \lambda_{f}\right)} \omega .
$$

Here the pair $\left(S(f, \lambda), f_{\lambda}\right)$ is a compact oriented branched suborbifold of $Z$ in which $S(f, \lambda)=\{z \in Z \mid \lambda(f(z))>0\}$ is the solution set equipped with the weight function $\lambda_{f}(z):=\lambda(f(z))$.

Proof In view of Theorem 4.14 the map $z \mapsto \lambda_{f}(z)$ defines a compact oriented branched suborbifold of $Z$. Hence, by the results in [20], the integrals $\int_{\left(S(f, \lambda), \lambda_{f}\right)} \omega$ are well-defined real numbers. To show that they do not depend on the choice of $\lambda$ we choose a second transversal pair $\left(f, \lambda^{\prime}\right)$ whose $\mathrm{sc}^{+}$-multisection $\lambda^{\prime}$ is supported in $U$ and view $f$ as a Fredholm section of the strong polyfold bundle $W \rightarrow[0,1] \times Z$. Now we take a parametrized $\mathrm{sc}^{+}-$multisection $\lambda_{t}$ having its support in $[0,1] \times U$ such that the pairs $\left(f, \lambda_{t}\right)$ are transversal and connect the $\mathrm{sc}^{+}$-multisection $\lambda_{0}=\lambda$ with the $\mathrm{sc}^{+}$-multisection $\lambda_{1}=\lambda^{\prime}$. The disjoint union $S\left(f, \lambda^{\prime}\right) \bigsqcup-S(f, \lambda)$ is the boundary of the solution set $S\left(f, \lambda_{t}\right)=\left\{(t, z) \in[0,1] \times Z \mid \lambda_{t}(z)>0\right\}$ which is a compact oriented suborbifold of $[0,1] \times Z$. Stokes' theorem from [20] leads to

$$
\int_{\left(S(f, \lambda), \lambda_{f}\right)} \omega=\int_{\left(S\left(f, \lambda^{\prime}\right), \lambda_{f}^{\prime}\right)} \omega .
$$

Hence the right hand side of $(10)$ is indeed independent of the choice of the transversal pair $(f, \lambda)$ in $U$ and hence $\Phi_{f}([\omega])$ is well defined by the formula (10). The proof of Theorem 4.23 is complete.

Note that we have a distinguished 0 -form on the polyfold $Z$, namely the constant 1 -function. Assume that $f$ is a proper and oriented Fredholm section of the strong polyfold bundle $W \rightarrow Z$ and has Fredholm index 0 . Then $\Phi_{f}([1])$ is a rational number and is a version of a degree for oriented proper Fredholm sections of strong polyfold bundles. 
The application of Theorem 4.23 to the Gromov-Witten invariants sketched in the introduction is as follows. We consider the polyfold $Z$ whose elements are the equivalence classes $[(S, j, M, D, u)]$ introduced in Definition 1.8 and the strong polyfold bundle $W \rightarrow Z$ in Theorem 1.11. Let $\bar{\partial}_{J}$ be the sc-smooth component-proper Fredholm section of the bundle according to Theorem 1.12. Given the homology class $A \in H_{2}(Q, \mathbb{Z})$ of the closed symplectic manifold $Q$ and two integers $g, k \geq 0$, we look at the polyfold $Z_{g, k} \subset Z$ of equivalence classes $[(S, j, M, D, u)]$ in which the nodal Riemann surface $S$ has arithmetic genus $g$ and is equipped with $k$ marked points, and the map $u$ represents the homology class $A$ of the manifold $Q$. The evaluation maps $\mathrm{ev}_{i}: Z_{g, k} \rightarrow Q$ and $\sigma: Z_{g, k} \rightarrow \overline{\mathcal{M}}_{g, k}$ are defined in the introduction and allow to pull back the differential forms on $Q$ and on $\overline{\mathcal{M}}_{g, m}$ to obtain sc-differential forms on the polyfold $Z_{g, k}$. Wedging combinations of these forms together, we can integrate over the oriented solution set $(\mathfrak{M}, w)=\left(S\left(\bar{\partial}_{J}, \lambda\right), \lambda_{\bar{\partial}_{J}}\right)$ assuming that the pair $\left(\bar{\partial}_{J}, \lambda\right)$ is transversal. Here

$$
\mathfrak{M}=S\left(\bar{\partial}_{J}, \lambda\right)=\left\{z \in Z_{g, k} \mid \lambda\left(\bar{\partial}_{J} z\right)>0\right\}
$$

and the weight function $w=\lambda_{\bar{\partial}_{J}}: Z_{g, k} \rightarrow \mathbb{Q}^{+}$is defined by $\lambda \bar{\partial}_{J}(z)=\lambda\left(\bar{\partial}_{J} z\right)$. The pair $(\mathfrak{M}, w)$ is an oriented compact branched suborbifold of the polyfold $Z_{g, k}$. Hence, in view of the abstract Theorem 4.23, the Gromov-Witten invariants can be constructed by means of the map

$$
\Phi_{A, g, m}^{Q}: H^{*}(Q)^{\otimes k} \otimes H_{*}\left(\overline{\mathcal{M}}_{g, m}\right) \rightarrow \mathbb{R}
$$

defined by the formula

$$
\Psi_{A, g, k}^{Q}\left(\left[\alpha_{1}\right], \ldots,\left[\alpha_{k}\right] ;[\tau]\right)=\int_{(\mathfrak{M}, w)} \operatorname{ev}_{1}^{*}\left(\alpha_{1}\right) \wedge \ldots \wedge \operatorname{ev}_{k}^{*}\left(\alpha_{k}\right) \wedge \sigma^{*}(\operatorname{PD}(\tau))
$$

where $\alpha_{1}, \ldots, \alpha_{k} \in H^{*}(Q)$ and where $\tau \in H_{*}\left(\overline{\mathcal{M}}_{g, k}\right)$ and where PD denotes the Poincare dual. That the formula is independent of the choice of the $\mathrm{sc}^{+}$-multisection $\lambda$ follows from Theorem 4.23.

Finally, we shall complete the proof of Theorem 1.4.

Proof Using Theorem 4.19 we take a generic perturbation $\lambda$ so that the solution set $S=S(f, \lambda)$ is in good position to the boundary. Assume that $S$ is of dimension $n$. Then for $[\omega, \tau] \in H_{\mathrm{dR}}^{n}(Z, \partial Z)$ we have the well-defined integral $\int_{(S, w)} \omega-\int_{(\partial S, w)} \tau$.

If $t \rightarrow f_{t}$ is an sc- smooth proper oriented homotopy of Fredholm sections connecting $f_{0}$ with $f_{1}$ we can view

$$
F(t, x)=f_{t}(x)
$$


as a proper Fredholm section of $W$ pulled-back by the projection map $[0,1] \times Z \rightarrow Z$. Then we can fix an open neighborhood $U$ of the solution set $S(f)=\{z \in Z \mid f(z)=0\}$ and an auxiliary norm so that the pair $(N, U)$ controls compactness. Assume that $\left(f_{i}, \lambda_{i}\right)$ are two admissible perturbations so that the corresponding solution sets are in good position to the boundary. The homotopy $t \mapsto f_{t}$ can be perturbed generically by the same argument as before to find a homotopy $\lambda_{t}$, which for $t=0,1$ coincides with the perturbations we already have, so that away from the boundaries $\{0\} \times Z$ and $\{1\} \times Z$ the perturbed pair $\left(f_{t}, \lambda_{t}\right)$ is in good position to the boundary. Now the previous discussion about the behavior of our invariant under our homotopy finishes the proof of Theorem 1.4.

\section{Appendix}

In the following we explain some of the more technical results as well as some of the necessary background material.

\subsection{Natural representation of stabilizers}

We shall study the local structure of the morphism set of an ep-polyfold groupoid in more detail.

We choose an object $x_{0} \in X$. By the ep-assumption, its stabilizer group $\mathbf{X}\left(x_{0}\right)$ is a finite group and we denote it by $G_{x_{0}}$. The following theorem describes the structure of the morphism set near the isotropy group.

Theorem 5.1 Given an ep-groupoid $X$, an object $x_{0} \in X$, and an open neighborhood $V \subset X$ of $x_{0}$. Then there exist an open neighborhood $U \subset V$ of $x_{0}$, a group homomorphism

$$
\varphi: G_{x_{0}} \rightarrow \operatorname{Diff}_{\mathrm{sc}}(U), \quad g \mapsto \varphi_{g}=t_{g} \circ s_{g}^{-1},
$$

and an sc-smooth map

$$
\Gamma: G_{x_{0}} \times U \rightarrow \mathbf{X}
$$

having the following properties:

- $\Gamma\left(g, x_{0}\right)=g$.

- $s(\Gamma(g, y))=y$ and $t(\Gamma(g, y))=\varphi_{g}(y)$ for all $y \in U$ and $g \in G_{x_{0}}$.

- If $h: y \rightarrow z$ is a morphism connecting two objects $y, z \in U$, then there exists a unique $g \in G_{x_{0}}$ such that $\Gamma(g, y)=h$. 
In particular, every morphism between points in $U$ belongs to the image of the map $\Gamma$. We call the group homomorphism $\varphi: G_{X} \rightarrow \operatorname{Diff}_{\mathrm{sc}}(U)$ a natural representation of the stabilizer group $G_{x_{0}}$.

Proof For every $g \in G_{x_{0}}$ we choose two contractible open neighborhoods $N_{g}^{t}$ and $N_{g}^{s} \subset \mathbf{X}$ on which the target and source maps $t$ and $s$ are sc-diffeomorphisms onto some open neighborhood $U_{0} \subset X$ of $x_{0}$. Since the isotropy group $G_{x_{0}}$ is finite we can assume that the open sets $N_{g}^{t} \cup N_{g}^{s}$ for $g \in G_{x_{0}}$ are disjoint and define the disjoint open neighborhoods $N_{g} \subset \mathbf{X}$ of $g$ by

$$
N_{g}:=N_{g}^{t} \cap N_{g}^{s}, \quad g \in G_{x_{0}} .
$$

We abbreviate the restrictions of the source and target maps by

$$
s_{g}:=s \mid N_{g} \quad \text { and } \quad t_{g}:=t \mid N_{g} .
$$

Lemma 5.2 With the choices made above there exists an open neighborhood $U_{1} \subset U_{0}$ of $x_{0}$ so that every morphism $h \in \mathbf{X}$ with $s(h)$ and $t(h) \in U_{1}$ belongs to $N_{g}$ for some $g \in G_{x_{0}}$.

Proof Arguing indirectly we find a sequence $h_{k} \in \mathbf{X}$ with $h_{k} \notin N_{g}$ for all $g \in G_{x_{0}}$ and satisfying $s\left(h_{k}\right), t\left(h_{k}\right) \rightarrow x_{0}$ as $k \rightarrow \infty$. By the properness assumption of eppolyfolds there is a convergent subsequence $h_{k_{l}} \rightarrow h \in \mathbf{X}$. Necessarily $h \in G_{x_{0}}$ and hence $h \in N_{g}$ for some $g \in G_{x_{0}}$. This contradiction implies the lemma.

Lemma 5.3 If $U_{1}$ is the open neighborhood of $x_{0}$ guaranteed by Lemma 5.2, then there exists an open neighborhood $U_{2} \subset U_{1}$ of $x_{0}$ so that the open neighborhood $U$ of $x_{0}$, which is defined as the union

$$
U:=\bigcup_{g \in G_{x_{0}}} t_{g} \circ s_{g}^{-1}\left(U_{2}\right),
$$

is contained in $U_{1}$ and invariant under all the maps $t_{g} \circ s_{g}^{-1}$ for $g \in G_{x_{0}}$.

Proof We choose an open neighborhood $U_{2} \subset U_{1}$ of $x_{0}$ so small that the union $U$ and also $t_{g} \circ s_{g}^{-1}(U)$ are contained in $U_{1}$ for all $g \in G_{x_{0}}$. Consider the map $t_{g} \circ s_{g}^{-1}: U \rightarrow X$ and choose $x \in U$. Then we can represent it as $x=t_{h} \circ s_{h}^{-1}(u)$ for some $h \in G_{x_{0}}$ and some $u \in U_{2}$. Now, $v:=t_{g} \circ s_{g}^{-1} \circ t_{h} \circ s_{h}^{-1}(u)$ belongs to $U_{1}$ and the formula implies the existence of a morphism $u \rightarrow v$ in $\mathbf{X}$. By Lemma 5.2 the morphism has necessarily the form $v=t_{g^{\prime}} \circ s_{g^{\prime}}^{-1}(u)$ for some $g^{\prime} \in G_{x_{0}}$. Since $u \in U_{2}$ it follows that $v=t_{g} \circ s_{g}^{-1}(x) \in U$ implying the desired invariance of $U$. 
In view of Lemma 5.3 we can associate with every $g \in G_{\chi_{0}}$ the sc-diffeomorphism

$$
\varphi(g):=t_{g} \circ s_{g}^{-1}: U \rightarrow U
$$

of the open neighborhood $U$ of $x_{0}$, and obtain the mapping

$$
\varphi: G_{x_{0}} \rightarrow \operatorname{Diff}_{\mathrm{sc}}(U), \quad g \mapsto \varphi_{g}
$$

Since the neighborhoods $N_{g} \subset \mathbf{X}$ of $g$ are disjoint and since the structure maps are continuous we conclude that $\varphi$ is a homomorphism of groups, in the following called the natural representation of the stabilizer group $G_{x_{0}}$ by sc-diffeomorphisms of the open neighborhood $U \subset X$ of $x_{0}$. Then we define

$$
\Gamma: G_{x_{0}} \times U \rightarrow \mathbf{X}, \quad \Gamma(g, y)=s_{g}^{-1}(y) .
$$

Summing up the consequences of Lemmas 5.2 and 5.3 we have proved Theorem 5.1. $\square$

The following structure theorem is fundamental for the constructions of perturbations.

Theorem 5.4 Every object $x_{0} \in X$ of an ep-groupoid possesses an open neighborhood $U \subset X$ having the following properties:

- On $U$, the stabilizer group $G_{x_{0}}$ has the natural representation

$$
\varphi: G_{x_{0}} \rightarrow \operatorname{Diff}_{\mathrm{sc}}(U) .
$$

- Assume $y_{0} \in X$ is an object for which there exists no morphism $y_{0} \rightarrow x$, where $x$ in $\bar{U}$. Then there exists an open neighborhood $V$ of $y_{0}$ so that for every $z \in V$ there is no morphism to an element in $\bar{U}$.

- Assume $y_{0} \in X$ is an object for which there exists no morphism $y_{0} \rightarrow x$ for every $x \in U$, but there exists a morphism to some element in $\bar{U}$. Then given an open neighborhood $W$ of $\partial U$ (the set theoretic boundary of $U$ ), there exists an open neighborhood $V$ of $y_{0}$ so that if there is a morphism $y \rightarrow x$ for some $y \in V$ and $x \in U$, then $x \in W$.

Moreover, the open set $U$ can be taken as small as we wish.

Proof For the first statement we refer to Theorem 5.1. We can choose the neighborhood $U$ of $x_{0}$ as small as we wish. Hence we may assume that

$$
t: s^{-1}(\bar{U}) \rightarrow X
$$

is proper. To prove the second statement we assume for $y_{0} \in X$ that there is no morphism to any element in $\bar{U}$. If no neighborhood $V$ with the desired properties 
exist, we find sequences $y_{k} \rightarrow y_{0}$ and $x_{k} \in \bar{U}$ so that there exist morphisms $y_{k} \rightarrow x_{k}$. Inverting these morphisms we obtain a sequence of morphisms $g_{k}: x_{k} \rightarrow y_{k}$, and the sequence of points in $X$,

$$
y_{k}=t\left(g_{k}\right) \in t\left(s^{-1}(\bar{U})\right) .
$$

By the properness assumption we may assume without loss of generality that $g_{k} \rightarrow g \in \mathbf{X}$ implying $x_{k} \rightarrow x^{\prime}$ in $X$ for some $x^{\prime}$. Therefore,

$$
g: x^{\prime} \rightarrow y_{0} \quad \text { and } \quad x^{\prime} \in \bar{U} .
$$

This contradiction proves the second assertion.

Assume, finally, that there exists no morphism $y_{0} \rightarrow x$ for $x \in U$, but a morphism $y_{0} \rightarrow \bar{x} \in \bar{U}$. Pick an arbitrary open neighborhood $W$ of $\partial U$. If $V$ with the desired properties does not exist we find sequences of morphisms $y_{k} \rightarrow y_{0}$ and elements $x_{k} \in U \backslash W$ admitting morphisms $g_{k}: y_{k} \rightarrow x_{k}$. Using the properness assumption again we may assume that $g_{k} \rightarrow g$ in $\mathbf{X}$ where $g: y_{0} \rightarrow x$ and $x \in U$, giving a contradiction. The proof of Theorem 5.4 is complete.

\subsection{Sc-smooth partitions of unity}

In this section we prove the existence of an sc-smooth partition of unity on an epgroupoid. We consider an ep-groupoid whose sc-structure is based on separable sc-Hilbert spaces. But we would like to point out that what is really needed is that the ep-groupoids are modeled on sc-scales $\left(E_{j}\right)_{j \geq 0}$ in which only the space $E_{0}$ is required to be a separable Hilbert space.

We view $[0,1]$ as a category with only the identity morphisms. An sc-smooth functor $f: X \rightarrow[0,1]$ on $X$ is an sc-smooth map on the object M-polyfold which is invariant under morphisms, that is, $f(x)=f(y)$ if there exists a morphism $h: x \rightarrow y$.

Definition 5.5 Let $X$ be an ep-groupoid and let $\mathcal{U}=\left(U_{\alpha}\right)_{\alpha \in A}$ be an open cover of $X$ consisting of saturated sets. An sc-partition of unity $\left(g_{\alpha}\right)_{\alpha \in A}$ subordinate to $\mathcal{U}$ consists of the locally finite collection of sc-smooth functors $g_{\alpha}: X \rightarrow[0,1]$ satisfying $\sum_{\alpha \in A} g_{\alpha}=1$ and $\operatorname{supp} g_{\alpha} \subset U_{\alpha}$ for every $\alpha \in A$.

The existence of an sc-smooth partition of unity depends on a sufficient supply of sc-smooth functions. We shall make use of the following result for separable Hilbert spaces proved in Fathi [5].

Lemma 5.6 Let $U$ and $W$ be open subsets of a separable Hilbert space $H$ such that $\bar{W} \subset U$. Then there exists a smooth function $f: H \rightarrow[0,1]$ having its support contained in $U$ and satisfying $f=1$ on $\bar{W}$. 
The proof of Lemma 5.6 extends easily to the case in which $U$ and $W$ are open subsets of a partial quadrant in a separable Hilbert space. In the next lemma we extend Lemma 5.6 to the sc-setting.

Lemma 5.7 Let $U$ and $W$ be open subsets of a splicing core $K$ such that $\bar{W} \subset U$. Then there exists an sc-smooth function $f: K \rightarrow[0,1]$ such that $f$ has its support in $U$ and is equal to 1 on $W$.

Proof Assume that $K=K^{\mathcal{S}}=\left\{(v, e) \in V \oplus E \mid \pi_{v}(e)=e\right\}$ is the splicing core associated with the splicing $\mathcal{S}=(\pi, E, V)$. Here $V$ is an open subset of a partial cone $C$ in a separable sc-Hilbert space $Z, E$ is a separable sc-Hilbert space, and $\pi: V \oplus E \rightarrow E$ is an sc-smooth map such that $\pi(v, \cdot):=\pi_{v}: E \rightarrow E$ is a bounded linear projection for every $v \in V$. Consider $\Phi: W \oplus E \rightarrow W \oplus E$ defined by $\Phi(v, e)=(v, \pi(v, e))$. The map $\Phi$ is sc-smooth and, in particular, continuous from level 0 to level 0 of $V \oplus E$. Moreover, $\Phi(V \oplus E)=K$. Put $W^{\prime}=\Phi^{-1}(W)$ and $U^{\prime}=\Phi^{-1}(U)$. Then $W^{\prime}$ and $U^{\prime}$ are open and since $\Phi^{-1}(\bar{W})$ is closed, we get $\overline{W^{\prime}}=\overline{\Phi^{-1}(W)} \subset \Phi^{-1}(\bar{W}) \subset U^{\prime}$. By Lemma 5.6, there exists a smooth function $f_{0}: V \oplus E \rightarrow[0,1]$ such that $\operatorname{supp} f_{0} \subset U^{\prime}$ and $f=1$ on $W^{\prime}$. Since $f_{0}$ is scsmooth in view of Proposition 2.15 in [21] and since the map $\pi$ is sc-smooth, the composition $f_{0} \circ \pi$ is also sc-smooth by the chain rule [21, Theorem 2.16]. Hence, putting $f:=f_{0} \mid K$, we obtain an sc-smooth function defined on $K$ having its support in $U$ and equal to 1 on $W$.

If $g: X \rightarrow[0,1]$ is an sc-smooth functor on the ep-groupoid $X$, we denote by $|g|$ the continuous function defined on the orbit space $|X|$ by $|g|(|x|):=g(x)$. Now we come to the statement of the main theorem of this section.

Theorem 5.8 (Sc-smooth partition of unity) Let $X$ be an ep-groupoid whose scstructure is based on separable sc-Hilbert spaces, and let $\mathcal{O}=\left(O_{\alpha}\right)_{\alpha \in A}$ be an open cover of the orbit space $|X|$. Then there exists an sc-smooth partition of unity $\left(g_{\alpha}\right)_{\alpha \in A}$ on $X$ so that the associated continuous partition of unity $\left(\left|g_{\alpha}\right|\right)_{\alpha \in A}$ of $|X|$ is subordinate to $\mathcal{O}=\left(O_{\alpha}\right)_{\alpha \in A}$.

The proof of Theorem 5.8 will follow from the next two lemmata which make us of Theorem 5.4 in the Appendix, Section 5.1.

Lemma 5.9 Let $\mathcal{O}=\left(O_{\alpha}\right)_{\alpha \in A}$ be an open cover of $|X|$. Then there exist locally finite open covers $\left(W_{j}\right)_{j \in J}$ and $\left(U_{j}\right)_{\beta \in J}$ subordinate to $\mathcal{O}$ and such that $\overline{W_{j}} \subset U_{j}$. The sets $W_{j}$ and $U_{j}$ are invariant under the natural representations of the isotropy groups $G_{x_{j}}$ on $U_{j}$ for some $x_{j} \in U_{j}$, and the open cover $\left(\pi^{-1}\left(\pi\left(U_{j}\right)\right)_{j \in J}\right.$ is locally finite. 
Proof In view of the paracompactness of $|X|$, there is a locally finite refinement $\left(Q_{\alpha}\right)_{\alpha \in A}$ of the cover $\left(O_{\alpha}\right)_{\alpha \in A}$. Then $\left(\pi^{-1}\left(Q_{\alpha}\right)\right)_{\alpha \in A}$ is a locally finite refinement of $\left(\pi^{-1}\left(O_{\alpha}\right)\right)_{\alpha \in A}$. For every point $x \in X$, we choose an open neighborhood $V_{x}$ intersecting only a finite number of sets $\pi^{-1}\left(Q_{\alpha}\right)$. We replace $V_{x}$ by its intersection with those $\pi^{-1}\left(Q_{\alpha}\right)$ which contains the point $x$. Observe that there is no morphism between the point $x$ and the sets $\pi^{-1}\left(Q_{\alpha}\right)$ which don't intersect $V_{x}$. Hence, shrinking $V_{x}$ further, we may assume that $V_{x}$ has the properties listed in Theorem 5.4 and that there are no morphisms between points in $V_{x}$ and points in the sets $\pi^{-1}\left(Q_{\alpha}\right)$ not intersecting $V_{x}$. The collection $\left(V_{x}\right)_{x \in X}$ is an open cover of $X$ and since $X$ is paracompact, there exists a locally finite refinement $\left(U_{j}^{\prime}\right)_{j \in J}$ of $\left(V_{x}\right)_{x \in X}$. For every $j \in J$, choose a point $x(j)$ such that $U_{j}^{\prime} \subset V_{x(j)}$. We abbreviate by $G_{j}$ the isotropy group $G_{x(j)}$ acting on $V_{x(j)}$ by its natural representation. We claim that $\left(\pi^{-1}\left(\pi\left(U_{j}^{\prime}\right)\right)\right)_{j \in J}$ is a locally finite cover of $X$. Indeed, take $y \in X$. Then $y \in U_{k}^{\prime} \subset V_{x(k)}$ for some $k \in J$. Since $\left(U_{j}^{\prime}\right)_{j \in J}$ is locally finite, there exists an open neighborhood $W_{y}$ of $y$ contained in $U_{k}^{\prime}$ and intersecting only a finite number of the sets $U_{j}^{\prime}$, say $U_{j_{1}}^{\prime}, \ldots, U_{j_{N}}^{\prime}$. Hence $k=j_{i}$ for some $1 \leq i \leq N$. Replacing $W_{y}$ by a smaller set, we may assume that $W_{y}$ is $G_{k}$-invariant. Assume that $z \in W_{y} \cap \pi^{-1}\left(\pi\left(U_{j}^{\prime}\right)\right)$ for some $j \neq j_{1}, \ldots, j_{N}$. Then there is a morphism between some point $v \in V_{j}$ and $z=\varphi_{g}(v)$ for some $g \in G_{j}$. In view of the definition of $V_{x(k)}$, we have $v \in V_{x(k)}$. Hence there is $h \in G_{k}$ such that $v=\varphi_{h}(z)$, and since $z \in W_{y}$ and $W_{y}$ is $G_{j}$-invariant $v \in W_{y}$. Consequently, $W_{y} \cap U_{j}^{\prime} \neq \varnothing$ and it follows that $W_{y}$ intersects only the sets $\pi^{-1}\left(\pi\left(U_{j_{1}}^{\prime}\right)\right), \ldots, \pi^{-1}\left(\pi\left(U_{j_{N}}^{\prime}\right)\right)$. For every $j \in J$, set $U_{j}=\bigcup_{g \in G_{j}} \varphi_{g}\left(U_{j}^{\prime}\right)$. Then $\pi^{-1}\left(\pi\left(U_{j}\right)\right)=\pi^{-1}\left(\pi\left(U_{j}^{\prime}\right)\right)$ and since the isotropy groups $G_{j}$ are finite, it follows that $\left(U_{j}\right)_{j \in J}$ is a locally finite cover of $X$ such that $U_{j} \subset V_{x(j)}$. Using paracompactness of $X$ again, we find a locally finite cover $\left(W_{j}^{\prime}\right)_{j \in J}$ such that $\frac{W_{j}^{\prime}}{W_{j}} U_{j}$. Define $\frac{W_{j}}{W_{j}}=\bigcup_{g \in G_{j}} \varphi_{g}\left(W_{j}^{\prime}\right)$. Then $W_{j}$ is a $G_{j}$-invariant open subset of $V_{j}$ such that $\overline{W_{j}} \subset U_{j}$, and the open cover $\left(W_{j}\right)_{j \in J}$ is locally finite. This completes the proof of the lemma.

Lemma 5.10 Let $U=U\left(x_{0}\right) \subset X$ be an open neighborhood of $x_{0}$ with the properties as listed in Theorem 5.4 and let $\varphi: U \rightarrow K^{\mathcal{S}}$ be a coordinate chart onto an open subset of the splicing core $K^{\mathcal{S}}$. Assume that $W$ is a $G_{x_{0}}$-invariant open subset of $U$ such that $\bar{W} \subset U$. Then there exists an sc-functor $f: X \rightarrow[0,1]$ satisfying $f=1$ on $\bar{W}$ and supp $f \subset \pi^{-1}(\pi(U))$.

Proof We choose an open $G_{x_{0}}$-invariant set $V$ such that $\bar{W} \subset V \subset \bar{V} \subset U$. With the help of Lemma 5.7 and the chart $\varphi: U \rightarrow K^{\mathcal{S}}$, we find an sc-smooth function $f_{0}: X \rightarrow[0,1]$ satisfying supp $f_{0} \subset V$ and $f_{0}=1$ on $\bar{W}$. Define the function $f_{1}$ on 
$U$ by

$$
f_{1}(x)=\frac{1}{\sharp G_{x_{0}}} \sum_{g \in G_{x_{0}}} f_{0}\left(\varphi_{g}(x)\right), \quad x \in U .
$$

Then $f_{1}$ is sc-smooth as a finite sum of sc-smooth functions, $0 \leq f_{1} \leq 1$, and $f_{1}=1$ on $x \in \bar{W}$ since $\bar{W}$ is $G_{x_{0}}$-invariant. Since $f_{0}=0$ on $U \backslash \bar{V}$ and $U \backslash \bar{V}$ is $G_{x_{0}}$-invariant, it follows that also $f_{1}=0$ on $U \backslash \bar{V}$. In particular, $f_{1}=0$ on $\partial V$.

We extend $f_{1}$ to the function $f: X \rightarrow[0,1]$ as follows. If $x \in U$, then $f(x):=f_{1}(x)$. If there exists a morphism between $x$ and some point $y \in U$, then we set $f(x):=f_{1}(y)$. Finally, if there is no morphism between $x$ and a point in $U$, then we set $f(x):=0$.

Clearly, $f(x)=f(y)$ if there is a morphism $h: x \rightarrow y$. Note also that $f(x)=0$ for $x \in \partial U$. Indeed, if there are no morphisms between $x$ and points of $U$, then $f(x)=0$ by the definition of $f$. If there exists a morphism between $x$ and $y \in U$, then by Theorem 5.4, the point $x$ belongs to $U \backslash \bar{V}$, so that again $f(x)=f_{1}(y)=0$.

We already know that $f$ is sc-smooth on $U$. To show that it is sc-smooth on $X$, we take $x \in X \backslash U$ and consider the following cases. If there is no morphism between $x$ and a point in $\bar{U}$, in particular, there is no morphism between $x$ and a point in $U$, then $f(x)=0$. By part (b) of Theorem 5.4, $f=0$ on some open neighborhood $U_{x}$ of $x$ and so $f$ is sc-smooth on $U_{x}$.

Next assume that there exists a morphism $h: x \rightarrow y$ between the point $x$ and a point $y \in U$. According to the definition of $f, f(x)=f_{1}(y)$. We find two open neighborhoods $U_{x}$ and $U_{y}$ of $x$ and $y$ such that $U_{y} \subset U$ and $t \circ s^{-1}: U_{x} \rightarrow U_{y}$ is an sc-diffeomorphism. Then, $f=f_{1} \circ t \circ s^{-1}$ on $U_{x}$ and since the right hand side is an sc-smooth function, the function $f$ is sc-smooth on $U_{x}$.

In the last case, assume that there is no morphism between $x$ and points of $U$ but there is a morphism between $x$ and some point $y \in \partial U$. Then again we find open neighborhoods $U_{x}$ and $U_{y}$ of points $x$ and $y$ such that $t \circ s^{-1}: U_{x} \rightarrow U_{y}$ is an sc-diffeomorphism. By Theorem 5.4, we may take these neighborhoods so small that the following holds. If there exists a morphism between a point $y^{\prime} \in U_{y}$ and a point $z \in U$, then necessarily $z \in U \backslash W$. At $y^{\prime}$ we have $f\left(y^{\prime}\right)=0$ since $f_{1}=0$ on $U \backslash W$. If there are no morphism between $y^{\prime} \in U_{y}$ and points in $U$, then $f\left(y^{\prime}\right)=0$. Hence $f$ is equal to 0 on $U_{y}$ and since $f \mid U_{x}=\left(f \mid U_{y}\right) \circ\left(t \circ s^{-1}\right)$, we conclude that $f$ is equal to 0 on $U_{x}$. So we proved that the function $f$ is sc-smooth on $X$.

It remains to prove that supp $f \subset \pi^{-1}(\pi(U))$. At every point $x \notin \pi^{-1}(\pi(U))$, $f(x)=0$. Hence it is enough to show that for every $x \in \partial \pi^{-1}(\pi(U))$ there exists a neighborhood $U_{x}$ of $x$ such that $f=0$ on $U_{x}$. To see this, we prove that there is an open neighborhood $U_{x}$ of $x$ such that if there is a morphism between $x^{\prime} \in U_{x}$ and 
a point in $y \in U$, then $y \in U \backslash W$. Otherwise, we find a sequence $\left(x_{n}\right)$ converging to $x$, a sequence $\left(y_{n}\right) \subset W$, and a sequence $\left(h_{n}\right)$ of morphisms $h_{n}: y_{n} \rightarrow x_{n}$. Since the map $t: s^{-1}\left(\overline{U\left(x_{0}\right)}\right) \rightarrow X$ is proper, there is subsequence of the morphisms $\left(h_{n}\right)$ converging to the morphism $h$. This implies that the subsequence of $\left(y_{n}\right)$ converges to the point $y \in W$ and that $h: y \rightarrow x$, contradicting the fact that there are no morphisms between points in $\partial \pi^{-1}(\pi(U))$ and points in $U$. Hence $f=0$ on $U_{x}$ and this proves that $\operatorname{supp} f \subset \pi^{-1}(\pi(U))$.

Proof of Theorem 5.8 Let $\left(O_{\alpha}\right)_{\alpha \in A}$ be an open cover of $|X|$. In view of Lemma 5.9 , there are open covers $\left(W_{j}\right)_{j \in J}$ and $\left(U_{j}\right)_{j \in J}$ subordinate to $\left(\pi^{-1}\left(O_{\alpha}\right)\right)$ such that $\overline{W_{j}} \subset U_{j}$. Moreover, the sets $W_{j}$ and $U_{j}$ are invariant with respect to the natural representation of $U_{j}$ and the cover $\left(\pi^{-1}\left(\pi\left(U_{j}\right)\right)\right)_{j \in J}$ is locally finite. By Lemma 5.10, for every $j \in J$, there is an sc-smooth functor $f_{j}^{\prime}: X \rightarrow[0,1]$ which is equal to 1 on $\overline{W_{j}}$ and supp $f_{j}^{\prime} \subset \pi^{-1}\left(\pi\left(U_{j}\right)\right)$. Set $f^{\prime}=\sum_{j \in J} f_{j}^{\prime}$. In view of the local finiteness of $\left(\pi^{-1}\left(\pi\left(U_{j}\right)\right)\right)_{j \in J}$, the sum has only a finitely many nonzero terms in a neighborhood of each point and thus defines an sc-smooth function. Because $f_{j}=1$ on $W_{j}$ and every point of $X$ is in some $W_{j}$, the sum is also positive. Now define $f_{j}=f_{j}^{\prime} / f^{\prime}$. Then each $f_{j}$ is an sc-smooth functor such that supp $f_{j} \subset \pi^{-1}\left(\pi\left(U_{j}\right)\right)$. Finally, we may reindex our functions $f_{j}$ so that they are indexed by the indices in the set $A$. Since the cover $\left(U_{j}\right)_{j \in J}$ is a refinement of $\left(\pi^{-1}\left(O_{\alpha}\right)\right)$, we choose for each $j$ an index $\alpha(j)$ such that $U_{j} \subset \pi^{-1}\left(O_{\alpha(j)}\right)$. Then for each $\alpha \in A$, we define $g_{\alpha}=\sum_{j, \alpha(j)=\alpha} f_{j}$. If there is no index $j$ satisfying $\alpha(j)=\alpha$, we set $g_{\alpha}=0$. Every $g_{\alpha}$ is smooth and invariant under the morphisms, satisfies $0 \leq g_{\alpha} \leq 1$ and $\operatorname{supp} g_{\alpha} \subset \pi^{-1}\left(O_{\alpha}\right)$. In addition, $\sum_{\alpha \in A} g_{\alpha}=\sum_{j \in J} f_{j}=1$. Consequently, $\left(g_{\alpha}\right)_{\alpha \in A}$ is a desired sc-smooth partition of unity.

\subsection{Submanifolds of M-polyfolds}

In [21, Definition 3.19], we have introduced the concept of a strong finite dimensional submanifold of an M-polyfold. It carries the structure of a manifold in a natural way. In [22] we have introduced the more general notion of a finite dimensional submanifold which we recall here for the convenience of the reader. Again, submanifolds according to the new definition will have natural manifold structures and, moreover, strong finite dimensional submanifolds are also submanifolds according to the new definition. The manifold structures induced in both cases are the same.

Definition 5.11 Let $X$ be an M-polyfold and $M \subset X$ a subset equipped with the induced topology. The subset $M$ is called a finite dimensional submanifold of $X$ provided the following holds: 
- The subset $M$ lies in $X_{\infty}$.

- At every point $m \in M$ there exists an M-polyfold chart

$$
(U, \varphi,(\pi, E, V))
$$

where $m \in U \subset X$ and where $\varphi: U \rightarrow O$ is a homeomorphism satisfying $\varphi(m)=0$, onto the open neighborhood $O$ of 0 in the splicing core $K$ associated with the sc-smooth splicing $(\pi, E, V)$. Here $V$ is an open neighborhood of 0 in a partial quadrant $C$ of the sc-Banach space $W$. Moreover, there exists a finitedimensional smooth linear subspace $N \subset W \oplus E$ in good position to $C$ and a corresponding sc-complement $N^{\perp}$, an open neighborhood $Q$ of $0 \in C \cap N$ and an sc-smooth map $A$ : $Q \rightarrow N^{\perp}$ satisfying $A(0)=0, D A(0)=0$ so that the map

$$
\Gamma: Q \rightarrow W \oplus E, \quad q \rightarrow q+A(q)
$$

has its image in $O$ and the image of the composition $\Phi:=\varphi^{-1} \circ \Gamma: Q \rightarrow U$ is equal to $M \cap U$.

- The map $\Phi: Q \rightarrow M \cap U$ is a homeomorphism.

We call the map $\Phi: Q \rightarrow U$ a good parametrization of a neighborhood of $m \in M$ in $M$.

In other words, a subset $M \subset X$ of an M-polyfold $X$ consisting of smooth points is a submanifold if for every $m \in M$ there is a good parametrization of an open neighborhood of $m$ in $M$. The following proposition shows that the transition maps $\Phi \circ \Psi^{-1}$ defined by two good parametrizations $\Phi$ and $\Psi$ are smooth, so the inverses of the good parametrizations define an atlas of smoothly compatible charts. Consequently, a finite dimensional submanifold is in a natural way a manifold with boundary with corners.

Proposition 5.12 Any two parametrizations of a finite dimensional submanifold $M$ of the $M$-polyfold $X$ are smoothly compatible.

Proof Assume that $m_{0}:=\varphi^{-1}\left(q_{0}+A\left(q_{0}\right)\right)=\psi^{-1}\left(p_{0}+B\left(p_{0}\right)\right)$ for two good parametrizations. Since both good parameterizations are local homeomorphisms onto an open neighborhood of $m_{0}$ in $M$, we obtain a local homeomorphism $O\left(p_{0}\right) \rightarrow O\left(q_{0}\right)$, $p \mapsto q(p)$, where the domain and codomain are relatively open neighborhoods in partial quadrants. We have

$$
q(p)+A(q(p))=\varphi \circ \psi^{-1}(p+B(p)) .
$$


Recall that $q(p) \in N$ and $A\left(q(p) \in N^{\perp}\right.$, where $N \oplus N^{\perp}=W \oplus E$ is an sc-splitting. If $P: W \oplus E \rightarrow N$ is an sc-projection along $N^{\perp}$, then

$$
q(p)=P\left(\varphi \circ \psi^{-1}(p+B(p)) .\right.
$$

The map $p \rightarrow q(p)$ is sc-smooth as a composition of sc-smooth maps. However, since the domain and codomain lie in finite dimensional smooth subspaces, the map is smooth in the usual sense.

\subsection{Orientations and determinants}

We begin by recalling determinants of linear Fredholm operators. More details and proofs can be found in Donaldson and Kronheimer [4] and Floer and Hofer [6], and, particularly, relevant in our context, in our papers [22] and [17].

The determinant of a linear Fredholm operator $T: E \rightarrow E$ between two Banach spaces is the one-dimensional real vector space

$$
\operatorname{det}(T)=\left(\wedge^{\max } \operatorname{ker}(T)\right) \otimes\left(\wedge^{\max } \operatorname{cokern}(T)\right)^{*}
$$

where the star $*$ refers to the dual space. The orientation of the Fredholm operator $T$ is, by definition, the orientation of the vector space $\operatorname{det}(T)$. If $\Gamma: X \rightarrow \mathcal{F}(E, F)$ is a continuous family of Fredholm operators $T: E \rightarrow F$, then the bundle

$$
\operatorname{det}(\Gamma)=\bigcup_{x \in X}\{x\} \times \operatorname{det}(\Gamma(x)) \rightarrow X
$$

carries the structure of a topological line-bundle. The same result holds true if the domains and targets of the operators vary in vector bundles.

We shall consider now a Fredholm section $f$ of the strong polyfold bundle $p$ : $W \rightarrow Z$ and let $P: E \rightarrow X$ be a model for $p$ over the ep-groupoid $X$ and let the proper Fredholm section $F: X \rightarrow E$ be the corresponding representative of the section $f$. As shown in $[21 ; 22]$ there is a well-defined notion of a linearization of $F$ at a smooth point $x \in X$. It is a class of sc-Fredholm operator differing by $\mathrm{sc}^{+}$-operators and defined as follows. If $x$ is the smooth point, we take a germ of $\mathrm{sc}^{+}-$sections $s$ defined near $x$ and satisfying

$$
F(x)=s(x) .
$$

Then $(F-s)(x)=0$ so that the linearization $(F-s)^{\prime}(x): T_{x} X \rightarrow E_{x}$ is well-defined. It is a classical Fredholm operator and hence possesses the determinant $\operatorname{det}\left((F-s)^{\prime}(x)\right)$. It depends clearly on the choice of the $\mathrm{sc}^{+}-$section $s$. If $s_{1}$ is another such $\mathrm{sc}^{+}$-section, then the linearizations $(F-s)^{\prime}(x)$ and $\left(F-s_{1}\right)^{\prime}(x)$ differ only by an $\mathrm{sc}^{+}$-operator and, therefore, have the same Fredholm index. The space of all these linearizations 
at the point $x$ is a contractible convex space, and we denote the collection of the associated determinants by $\operatorname{DET}(F, x)$. Consequently, if the determinant of one of these Fredholm operators is oriented, then, by continuation, every other linearization becomes canonically oriented as well.

To describe the behavior of the determinants under the morphisms, we look at the morphism $\varphi: x \rightarrow y$ between two smooth points in $X$. We choose open neighborhoods $\mathbf{U}(\varphi) \subset \mathbf{X}$ of the morphism $\varphi$ and $U(x)$ and $U(y)$ of the points $x, y \in X$ (as small as necessary) such that the source and the target maps $s: \mathbf{U}(\varphi) \rightarrow U(x)$ and $t: \mathbf{U}(\varphi) \rightarrow$ $U(y)$ are sc-smooth diffeomorphisms. Then the morphism $\varphi$ extends to the scdiffeomorphism $\sigma: U(x) \rightarrow U(y)$ defined by $\sigma:=t \circ s^{-1}$ and satisfying $\sigma(x)=y$. With an sc ${ }^{+}$-germ $s_{0}: U(x) \rightarrow E$ satisfying $s_{0}(x)=F(x)$ we associate the $\mathrm{sc}^{+}$-germ $s_{1}: U(y) \rightarrow E$ defined by

$$
s_{1}(\sigma(z)):=\mu\left(s^{-1}(z), s_{0}(z)\right), \quad z \in U(x) .
$$

Since $F: X \rightarrow E$ is a functor, it satisfies the identity

$$
F(\sigma(z))=\mu\left(s^{-1}(z), F(z)\right), \quad z \in U(x)
$$

where $\mu: \mathbf{X}_{s} \times{ }_{p} E \rightarrow E$ is the strong bundle map defined Section 2.4. In particular, $F(y)=F(\sigma(y))=\mu(\varphi, F(x))=\mu\left(\varphi, s_{0}(x)\right)=s_{1}(y)$. Linearizing both sides of (11) at the point $x$ we find that

$$
\left(F-s_{1}\right)^{\prime}(y) \circ T \sigma(x) \cdot h=\mu\left(\varphi,\left(F-s_{0}\right)^{\prime}(x) \cdot h .\right.
$$

Abbreviating the linear isomorphism

$$
\Phi:=\mu(\varphi, \cdot): E_{x} \rightarrow E_{y}
$$

and recalling that, by definition $T \varphi=T \sigma(x)$, one obtains

$$
\Phi^{-1} \circ\left(F-s_{1}\right)^{\prime}(y) \circ T \varphi=\left(F-s_{0}\right)^{\prime}(x) .
$$

This formula shows that the morphism $\varphi: x \rightarrow y$ determines the natural isomorphism

$$
\varphi_{*}: \operatorname{det}\left(\left(F-s_{0}\right)^{\prime}(x)\right) \rightarrow \operatorname{det}\left(\left(F-s_{1}\right)^{\prime}(y)\right)
$$

between the determinants of the linearizations at the source and the target of $\varphi$.

To define the continuation of the orientations along an sc-smooth curve, we consider the sc-smooth path $\Theta:[0,1] \rightarrow X$ connecting the smooth point $\Theta(0)$ with the smooth point $\Theta(1)$. It is easy to construct an sc-smooth family of germs of $\mathrm{sc}^{+}-$sections $s_{t}$ satisfying

$$
s_{t}(\Theta(t))=F(\Theta(t))
$$


where $s_{t}$ is defined on an open neighborhood of the point $\Theta(t)$. Using the Fredholm property of the section $F$ one proves in [17], that the bundle

$$
\bigcup_{t \in[0,1]}\{t\} \times \operatorname{det}\left(\left(F-s_{t}\right)^{\prime}(\Theta(t))\right) \rightarrow[0,1]
$$

has in a natural way the structure of a real topological line-bundle over $[0,1]$. (We should point out that this is not entirely trivial because the linearizations do not depend continuously as bounded operators on the points $\Theta(t)$ at which the section is linearized.) As a consequence, we can define the continuation of the orientations of the linearizations along an sc-smooth path in $X$.

Definition 5.13 An orientation for the sc-Fredholm section $F$ of the strong bundle $P: E \rightarrow X$ consists of an orientation for the linearization of $F$ at every smooth point $x \in X$, which is invariant under the morphisms and stable under continuation along sc-smooth paths in $X$.

Two oriented sc-Fredholm sections $F$ of $P: E \rightarrow X$ and $F^{\prime}$ of $P^{\prime}: E^{\prime} \rightarrow X^{\prime}$ are called equivalent, if there is a common bundle refinement pulling back the sections to the same oriented section. Since bundle equivalences induce isomorphisms between the determinants, we can therefore define an orientation for a Fredholm section $f$ of a strong polyfold bundle $p: W \rightarrow Z$.

If $F$ is an oriented Fredholm section and $(F, \Lambda)$ is a transversal pair and $\Lambda(F(x))>0$, then the linearization at the smooth solution $x$ is an intrinsic finite collection of sc-Fredholm operators which all differ by $\mathrm{sc}^{+}$-operators. Therefore, we obtain an orientation for the manifolds of the local solution structure. In view of the compatibility with morphisms and the stability under continuation, the solution set inherits the structure of an oriented branched suborbifold. The details and the proofs will be carried out in [17].

\section{References}

[1] K Cieliebak, K Mohnke, Symplectic hypersurfaces and transversality in GromovWitten theory, J. Symplectic Geom. 5 (2007) 281-356 MR2399678

[2] K Cieliebak, I Mundet i Riera, D A Salamon, Equivariant moduli problems, branched manifolds, and the Euler class, Topology 42 (2003) 641-700 MR1953244

[3] S K Donaldson, Symplectic submanifolds and almost-complex geometry, J. Differential Geom. 44 (1996) 666-705 MR1438190 
[4] S K Donaldson, P B Kronheimer, The geometry of four-manifolds, Oxford Math. Monogr., Oxford Science Publ., The Clarendon Press, Oxford Univ. Press, New York (1990) MR1079726

[5] A Fathi, Partitions of unity for countable covers, Amer. Math. Monthly 104 (1997) 720-723 MR1476756

[6] A Floer, H Hofer, Coherent orientations for periodic orbit problems in symplectic geometry, Math. Z. 212 (1993) 13-38 MR1200162

[7] K Fukaya, Y-G Oh, H Ohta, K Ono, Lagrangian intersection Floer theory-anomaly and obstruction, Preprint

[8] K Fukaya, K Ono, Arnold conjecture and Gromov-Witten invariant, Topology 38 (1999) 933-1048 MR1688434

[9] P Gabriel, M Zisman, Calculus of fractions and homotopy theory, Ergebnisse series 35, Springer, New York (1967) MR0210125

[10] A Haefliger, Structures feuilletées et cohomologie à valeur dans un faisceau de groupoïdes, Comment. Math. Helv. 32 (1958) 248-329 MR0100269

[11] A Haefliger, Homotopy and integrability, from: "Manifolds-Amsterdam 1970 (Proc. Nuffic Summer School)”, Lecture Notes in Math. 197, Springer, Berlin (1971) 133-163 MR0285027

[12] A Haefliger, Groupoïdes d'holonomie et classifiants, from: "Transversal structure of foliations (Toulouse, 1982)”, Astérisque 116 (1984) 70-97 MR755163

[13] A Haefliger, Groupoids and foliations, from: "Groupoids in analysis, geometry, and physics (Boulder, CO, 1999)”, (A Ramsay, J Renault, editors), Contemp. Math. 282, Amer. Math. Soc. (2001) 83-100 MR1855244

[14] H Hofer, Lectures on polyfolds and applications I: Basic concepts and illustrations, to appear in Courant Lecture Note.

[15] H Hofer, K Wysocki, E Zehnder, Applications of polyfold theory I: The polyfolds of Gromov-Witten theory, in preparation

[16] H Hofer, K Wysocki, E Zehnder, Applications of polyfold theory II: The polyfolds of symplectic field theory, in preparation

[17] H Hofer, K Wysocki, E Zehnder, Connections and determinant bundles for polyfold Fredholm operators, in preparation

[18] H Hofer, K Wysocki, E Zehnder, The Fredholm property of the nonlinear CauchyRiemann operator in the polyfold set-up for symplectic field theory, in preparation

[19] H Hofer, K Wysocki, E Zehnder, Fredholm theory in polyfolds I: Functional analytic methods, book in preparation

[20] H Hofer, K Wysocki, E Zehnder, Integration theory for zero sets of polyfold Fredholm sections, submitted for publication arXiv:0711.0781 
[21] H Hofer, K Wysocki, E Zehnder, A general Fredholm theory. I. A splicing-based differential geometry, J. Eur. Math. Soc. 9 (2007) 841-876 MR2341834

[22] H Hofer, K Wysocki, E Zehnder, A general Fredholm theory II: Implicit function theorems, Geom. Funct. Anal. 18 (2009)

[23] J Li, G Tian, Virtual moduli cycles and Gromov-Witten invariants of general symplectic manifolds, from: "Topics in symplectic 4-manifolds (Irvine, CA, 1996)", (R Stern, editor), First Int. Press Lect. Ser. I, Int. Press, Cambridge, MA (1998) 47-83 MR1635695

[24] G Lu, G Tian, Constructing virtual Euler cycles and classes, Int. Math. Res. Surv. (2008) Art. ID rym001, 220pp. MR2369606

[25] Y I Manin, Frobenius manifolds, quantum cohomology, and moduli spaces, Amer. Math. Soc. Coll. Publ. 47, Amer. Math. Soc. (1999) MR1702284

[26] D McDuff, Groupoids, branched manifolds and multisections, J. Symplectic Geom. 4 (2006) 259-315 MR2314215

[27] D McDuff, D Salamon, J-holomorphic curves and symplectic topology, Amer. Math. Soc. Coll. Publ. 52, Amer. Math. Soc. (2004) MR2045629

[28] I Moerdijk, Orbifolds as groupoids: an introduction, from: "Orbifolds in mathematics and physics (Madison, WI, 2001)", (A Adem, J Morava, Y Ruan, editors), Contemp. Math. 310, Amer. Math. Soc. (2002) 205-222 MR1950948

[29] I Moerdijk, J Mrčun, Introduction to foliations and Lie groupoids, Cambridge Studies in Advanced Math. 91, Cambridge Univ. Press (2003) MR2012261

[30] Y Ruan, Symplectic topology on algebraic 3-folds, J. Differential Geom. 39 (1994) 215-227 MR1258920

[31] Y Ruan, Topological sigma model and Donaldson-type invariants in Gromov theory, Duke Math. J. 83 (1996) 461-500 MR1390655

[32] G Tian, Quantum cohomology and its associativity, from: "Current developments in mathematics, 1995 (Cambridge, MA)”, (R Bott, M Hopkins, A Jaffe, I Singer, D Stroock, S Yau, editors), Int. Press, Cambridge, MA (1994) 361-401 MR1474981

Courant Institute, New York University

251 Mercer Street, New York, 10012, USA

Mathematics Department, Penn State University

University Park, State College, PA 16802, USA

Department of Mathematik, ETH-Zentrum

CH 8092 Zürich, Switzerland

hofer@cims.nyu.edu, wysocki@math.psu.edu, eduard.zehnder@math.ethz.ch

Proposed: Leonid Polterovich

Seconded: Yasha Eliashberg, Danny Calegari
Received: 4 October 2008

Accepted: 22 April 2009 